Supporting Information for:

\title{
A rapid and efficient assay for the characterization of substrates and inhibitors of nicotinamide $N$-methyltransferase
}

\author{
Matthijs J. van Haren, ${ }^{\mathrm{a}}$ Javier Sastre Toraño, ${ }^{\mathrm{a}}$ Davide Sartini, ${ }^{\mathrm{b}}$ Monica Emanuelli, \\ Richard B. Parsons ${ }^{\mathrm{c}}$, Nathaniel I. Martin ${ }^{\mathrm{a}, *}$
}

${ }^{a}$ Department of Chemical Biology \& Drug Discovery, Utrecht Institute for Pharmaceutical Sciences, Utrecht University, Universiteitsweg 99, 3584 CG Utrecht, The Netherlands

${ }^{b}$ Department of Clinical Sciences, Universitá Politecnica delle Marche, Ancona, Italy

'Institute of Pharmaceutical Science, King's College London, London SE1 9NH, UK

\author{
Department of Chemical Biology \& Drug Discovery, \\ Utrecht Institute for Pharmaceutical Sciences, Utrecht University,
}

David de Wied Building, Office: 5.64, Universiteitsweg 99, 3584 CG Utrecht, The Netherlands.

n.i.martin@uu.nl

\section{Table of Contents}

\section{Page}

S2 General Procedures

S4-10 Experimental details and analytical data for compounds 1-11

S11-13 Curves of the Michaelis-Menten and substrate inhibition kinetics for compounds 1-11

S14-36 $\quad{ }^{1} \mathrm{H}$ and ${ }^{13} \mathrm{C}$ NMR spectra for compounds 1-11

S37 References 


\section{General procedures}

\section{Reagents and Instrumentation for characterization}

All reagents employed were of American Chemical Society (ACS) grade or finer and were used without further purification unless otherwise stated. For compound characterization ${ }^{1} \mathrm{H}$ NMR spectra were recorded at $400 \mathrm{MHz}$ with chemical shifts reported in parts per million (ppm) downfield relative to tetramethylsilane (TMS), $\mathrm{H}_{2} \mathrm{O}(\delta 4.79)$ or DMSO $(\delta 2.50) .{ }^{1} \mathrm{H}$ NMR data are reported in the following order: multiplicity (s, singlet; d, doublet; t, triplet; q, quartet and m, multiplet), coupling constant $(\mathrm{J})$ in hertz $(\mathrm{Hz})$ and the number of protons. Where appropriate, the multiplicity is preceded by br, indicating that the signal was broad. ${ }^{13} \mathrm{C}$ NMR spectra were recorded at $101 \mathrm{MHz}$ with chemical shifts reported relative to $\mathrm{CDCl}_{3}(\delta 77.16)$ or DMSO $(\delta 39.52)$. The ${ }^{13} \mathrm{C}$ NMR spectra of the compounds recorded in $\mathrm{D}_{2} \mathrm{O}$ could not be referenced. High-resolution mass spectrometry (HRMS) analysis was performed using a Q-TOF instrument.

\section{General procedure for the synthesis of reference standards and internal standards}<smiles></smiles>

$\mathrm{MW}=137.1$

$1 \mathrm{~b}$

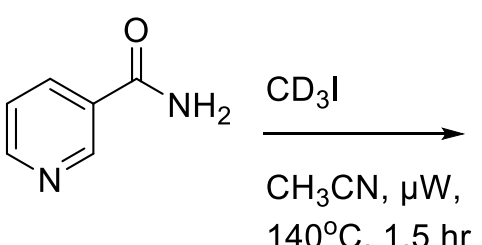

1<smiles></smiles>

$\mathrm{MW}=140.1$

$1 \mathrm{c}$

Scheme S1. General procedure used for the synthesis of reference standards (using methyl iodide), and internal standards (using trideuteromethyl iodide).

Substrate (1.0 eq, $1.0 \mathrm{mmol})$ is dissolved in $2 \mathrm{~mL}$ dry $\mathrm{CH}_{3} \mathrm{CN}$ and methyl iodide (2.2 eq, 2.2 mmol, $312 \mathrm{mg}$ ) or $\mathrm{d}_{3}$-methyl iodide $(2.2 \mathrm{eq}, 2.2 \mathrm{mmol}, 319 \mathrm{mg}$ ) is added. The tube is sealed and reacted in a microwave for 90 minutes at $140^{\circ} \mathrm{C}$. If necessary, the product is precipitated with dry diethyl ether. The precipitate is filtered off and washed with dry diethyl ether.

\section{Standards prepared via alternate procedures}<smiles>N#Cc1cccnc1</smiles>

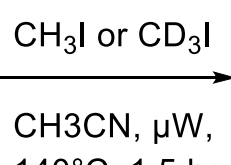
$140^{\circ} \mathrm{C}, 1.5 \mathrm{hr}$<smiles>[R][n+]1cccc(C#N)c1</smiles>

$\mathrm{R}=\mathrm{CH}_{3}$ or $\mathrm{CD}_{3}$

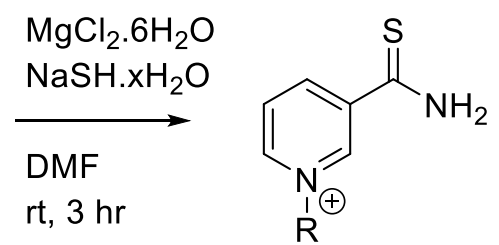

2b, $\mathrm{R}=\mathrm{CH}_{3}$

2c, $\mathrm{R}=\mathrm{CD}_{3}$

Scheme S2. Synthesis of thionicotinamide from $N^{\prime}$-methyl-3-pyridinecarbonitrile

$N^{\prime}$-methyl-thionicotinamide $\mathbf{2 b}$ and $N^{\prime}$ - $\mathrm{d}_{3}$-methyl-thionicotinamide $\mathbf{2 c}$ were synthesized from 3-pyridinecarbonitrile, which was first methylated using the general procedure and subsequently thionated using sodium hydrosulphide following a literature procedure. ${ }^{2}$ 


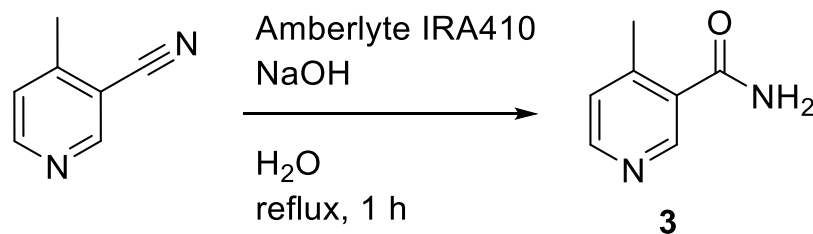

Scheme S3. Synthesis of 4-methylnicotinamide by alkaline hydrogenolysis using Amberlyte IRA410 resin

4-methylnicotinamide 3 is prepared by alkaline hydrogenolysis of 4-methyl-3-cyanopyridine using alkaline ion-exchange resin Amberlite IRA 410 as previously described. ${ }^{1}$ The standards of $\mathbf{3}$ are prepared using the general procedure.
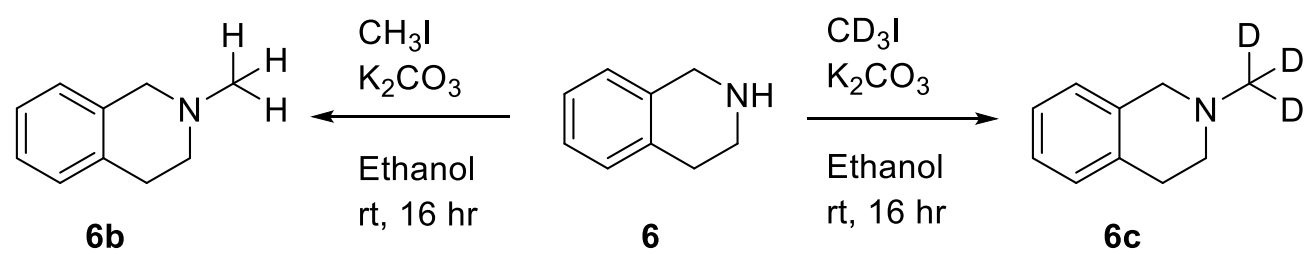

Scheme S4. Synthesis of the standards of 1,2,3,4-tetrahydroisoquinoline

1,2,3,4-tetrahydroisoquinoline $\mathbf{6}$ is methylated using methyl iodide or $\mathrm{d}_{3}$-methyl iodide and potassium carbonate in ethanol at room temperature overnight and subsequent purification by column chromatography.<smiles>COc1cccc[n+]1C=O</smiles>

$11 b$

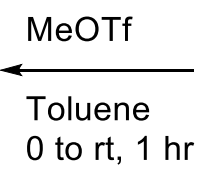

0 to $\mathrm{rt}, 1 \mathrm{hr}$<smiles>COc1ccccn1</smiles>

11

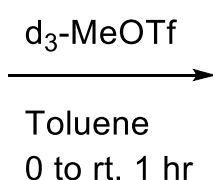

0 to $\mathrm{rt}, 1 \mathrm{hr}$

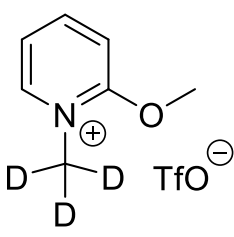

$11 \mathrm{c}$

Scheme S5. Synthesis of the standards of 2-methoxypyridine using methyltriflate

$N$-methyl-2-methoxypyridine $\mathbf{1 1 b}$ was synthesized by reaction with methyl triflate as previously described. ${ }^{3} \mathrm{~N}-\mathrm{d}_{3}$-methyl-2-methoxypyridine 11c was synthesized in a similar manner by reaction with $d_{3}$-methyl triflate, which was freshly prepared from $d_{3}$-methyl iodide and silver triflate. 


\section{3-carbamoyl-1-methylpyridin-1-ium iodide 1b}<smiles></smiles>

Obtained from commercial sources.

3-carbamoyl-1-(methyl-d3)pyridin-1-ium iodide 1c<smiles></smiles>

Synthesized according to the general procedure.

${ }^{1} \mathrm{H}$ NMR $\left(400 \mathrm{MHz}, \mathrm{DMSO}-d_{6}\right) \delta 9.36(\mathrm{~s}, 1 \mathrm{H}), 9.07(\mathrm{~d}, J=5.9 \mathrm{~Hz}, 1 \mathrm{H}), 8.87(\mathrm{~d}, J=8.0 \mathrm{~Hz}, 1 \mathrm{H})$, $8.47(\mathrm{~s}, 1 \mathrm{H}), 8.21(\mathrm{t}, J=7.0 \mathrm{~Hz}, 1 \mathrm{H}), 8.10(\mathrm{~s}, 1 \mathrm{H})$.

${ }^{13} \mathrm{C}$ NMR $\left(101 \mathrm{MHz}\right.$, DMSO- $\left.d_{6}\right) \delta 163.26,147.60,146.10,143.26,133.70,127.83$

HRMS (ESI): calculated for $\mathrm{C}_{7} \mathrm{H}_{6} \mathrm{D}_{3} \mathrm{~N}_{2} \mathrm{O}^{+} 140.0898$, found 140.0883 .

\section{3-cyano-1-methylpyridin-1-ium iodide}

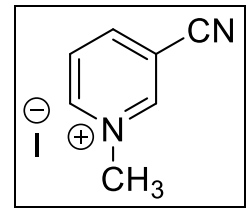

Synthesized according to the general procedure.

${ }^{1} \mathrm{H}$ NMR (400 MHz, DMSO- $\left.d_{6}\right) \delta 9.74(\mathrm{~s}, 1 \mathrm{H}), 9.25(\mathrm{~d}, J=5.4 \mathrm{~Hz}, 1 \mathrm{H}), 9.07(\mathrm{~d}, J=7.9 \mathrm{~Hz}, 1 \mathrm{H})$, $8.34(\mathrm{t}, J=6.7 \mathrm{~Hz}, 1 \mathrm{H}), 4.37(\mathrm{~s}, 3 \mathrm{H})$.

${ }^{13} \mathrm{C}$ NMR $\left(101 \mathrm{MHz}, \mathrm{DMSO}-d_{6}\right) \delta 149.69,149.08,148.07,127.95,113.87,112.11,48.61$. HRMS (ESI): calculated for $\mathrm{C}_{7} \mathrm{H}_{7} \mathrm{~N}_{2}{ }^{+}$119.0604, found 119.0615 .

\section{3-cyano-1-(methyl-d3)pyridin-1-ium iodide}

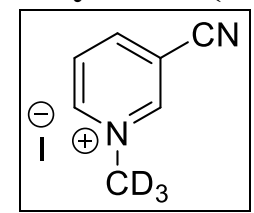

Synthesized according to the general procedure.

${ }^{1} \mathrm{H}$ NMR (400 MHz, DMSO- $\left.d_{6}\right) \delta 9.74(\mathrm{~s}, 1 \mathrm{H}), 9.25(\mathrm{~d}, J=5.3 \mathrm{~Hz}, 1 \mathrm{H}), 9.07(\mathrm{~d}, J=7.6 \mathrm{~Hz}, 1 \mathrm{H})$, $8.34(\mathrm{t}, J=6.4 \mathrm{~Hz}, 1 \mathrm{H})$.

${ }^{13} \mathrm{C}$ NMR (101 MHz, DMSO- $\left.d_{6}\right) \delta 149.73,149.08,148.09,127.95,113.87,112.11$. HRMS (ESI): calculated for $\mathrm{C}_{7} \mathrm{H}_{4} \mathrm{D}_{3} \mathrm{~N}_{2}{ }^{+} 122.0792$, found 122.0810 . 
<smiles></smiles>

1-methyl-3-cyanopyridinium iodide ( $246 \mathrm{mg}, 1.0 \mathrm{mmol}, 1.0 \mathrm{eq}$ ) is added to a slurry of $\mathrm{NaSH}_{\mathrm{xH}} \mathrm{O}$ (240 mg, $3.0 \mathrm{mmol}, 3.0 \mathrm{eq}, 70 \% \mathrm{NaSH}$ ) and $\mathrm{MgCl}_{2} .6 \mathrm{H}_{2} \mathrm{O}$ (305 mg, $1.5 \mathrm{mmol}, 1.5 \mathrm{eq}$ ) in DMF (3 $\mathrm{mL}$ ). The mixture is stirred for 3 hours at room temperature. The precipitate is filtered off and the mixture is concentrated under vacuum. The residue is dissolved in water, filtered and freeze dried yielding the $\mathbf{2 b}$ as an orange red solid.

${ }^{1} \mathrm{H}$ NMR (400 MHz, DMSO-d $\left.{ }_{6}\right) \delta 10.75-10.05(\mathrm{~m}, 2 \mathrm{H}), 9.44(\mathrm{~s}, 1 \mathrm{H}), 9.05(\mathrm{~d}, J=5.9 \mathrm{~Hz}, 1 \mathrm{H}), 8.83$ (d, $J=8.4 \mathrm{~Hz}, 1 \mathrm{H}), 8.16(\mathrm{t}, J=7.1 \mathrm{~Hz}, 1 \mathrm{H}), 4.39$ (s, 3H).

${ }^{13} \mathrm{C}$ NMR $\left(101 \mathrm{MHz}, \mathrm{DMSO}-d_{6}\right) \delta 192.72,146.44,144.69,141.95,138.27,126.91,48.21$. HRMS (ESI): calculated for $\mathrm{C}_{7} \mathrm{H}_{9} \mathrm{~N}_{2} \mathrm{~S}^{+} 153.0481$, found 153.0449 .

\section{3-carbamothioyl-1-(methyl-d3)pyridin-1-ium iodide 2c}

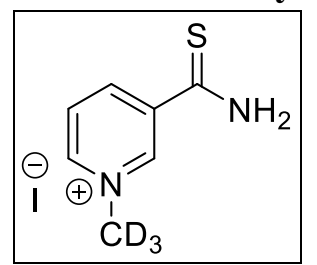

Prepared as described for compound $\mathbf{2 b}$.

${ }^{1} \mathrm{H}$ NMR (400 MHz, DMSO- $d_{6}$ ) $\delta 10.75-10.05(\mathrm{~m}, 2 \mathrm{H}), 9.43(\mathrm{~s}, 1 \mathrm{H}), 9.05(\mathrm{~d}, J=6.1 \mathrm{~Hz}, 1 \mathrm{H}), 8.83$ (d, $J=8.4 \mathrm{~Hz}, 1 \mathrm{H}), 8.16(\mathrm{dd}, J=8.0,6.3 \mathrm{~Hz}, 1 \mathrm{H})$.

${ }^{13} \mathrm{C}$ NMR (101 MHz, DMSO- $\left.d_{6}\right) \delta 192.70,146.44,144.75,141.95,138.31,126.92$.

HRMS (ESI): calculated for $\mathrm{C}_{7} \mathrm{H}_{6} \mathrm{D}_{3} \mathrm{~N}_{2} \mathrm{~S}^{+} 156.0669$, found 156.0626 .

\section{3-carbamoyl-1,4-dimethylpyridin-1-ium iodide 3b}<smiles></smiles>

Synthesized according to the general procedure.

${ }^{1} \mathrm{H}$ NMR (400 MHz, DMSO-d $) \delta 9.06(\mathrm{~s}, 1 \mathrm{H}), 8.87(\mathrm{~d}, J=6.2 \mathrm{~Hz}, 1 \mathrm{H}), 8.19(\mathrm{~s}, 1 \mathrm{H}), 8.11(\mathrm{~s}, 1 \mathrm{H})$, $8.05(\mathrm{~d}, J=6.3 \mathrm{~Hz}, 1 \mathrm{H}), 4.29$ (s, 2H), 2.63 (s, 2H).

${ }^{13} \mathrm{C}$ NMR (101 MHz, DMSO- $\left.d_{6}\right) \delta 164.74,155.59,144.77,143.43,135.46,129.12,47.23,19.85$. HRMS (ESI): calculated for $\mathrm{C}_{8} \mathrm{H}_{11} \mathrm{~N}_{2} \mathrm{O}^{+} 151.0866$, found 151.0910 . 


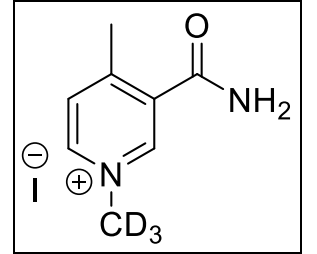

Synthesized according to the general procedure.

${ }^{1} \mathrm{H}$ NMR (400 MHz, DMSO-d $\left.d_{6}\right) \delta 9.06(\mathrm{~s}, 1 \mathrm{H}), 8.87(\mathrm{~d}, J=6.3 \mathrm{~Hz}, 1 \mathrm{H}), 8.20(\mathrm{~s}, 1 \mathrm{H}), 8.11(\mathrm{~s}, 1 \mathrm{H})$, $8.05(\mathrm{~d}, J=6.3 \mathrm{~Hz}, 1 \mathrm{H}), 2.63(\mathrm{~s}, 3 \mathrm{H})$.

${ }^{13} \mathrm{C}$ NMR (101 MHz, DMSO- $\left.d_{6}\right) \delta 164.74,155.61,144.75,143.42,135.43,129.11,19.85$.

HRMS (ESI): calculated for $\mathrm{C}_{8} \mathrm{H}_{8} \mathrm{D}_{3} \mathrm{~N}_{2} \mathrm{O}^{+} 154.1054$, found 154.1077 .

\section{2-methylisoquinolin-2-ium iodide $4 b$}

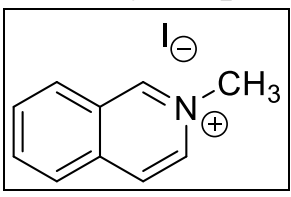

Synthesized according to the general procedure.

${ }^{1} \mathrm{H}$ NMR $\left(400 \mathrm{MHz}, \mathrm{d}_{2} \mathrm{O}\right) \delta 9.61(\mathrm{~s}, 1 \mathrm{H}), 8.42(\mathrm{~d}, J=6.6 \mathrm{~Hz}, 1 \mathrm{H}), 8.38-8.27(\mathrm{~m}, 2 \mathrm{H}), 8.28-8.09$ (m, 2H), 7.99 (t, $J=7.1 \mathrm{~Hz}, 1 \mathrm{H}), 4.52(\mathrm{~s}, 3 \mathrm{H})$.

${ }^{13} \mathrm{C}$ NMR (101 MHz, $\left.\mathrm{d}_{2} \mathrm{O}\right) \delta 149.70,137.02,136.84,134.79,131.22,129.80,127.33,127.04,126.04$, 47.94.

HRMS (ESI): calculated for $\mathrm{C}_{10} \mathrm{H}_{10} \mathrm{~N}^{+} 144.0808$, found 144.0785.

\section{2-(methyl-d3)isoquinolin-2-ium iodide 4c}

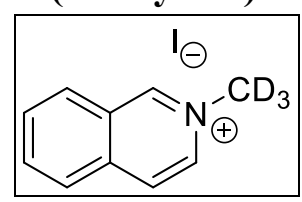

Synthesized according to the general procedure.

${ }^{1} \mathrm{H}$ NMR $\left(400 \mathrm{MHz}, \mathrm{d}_{2} \mathrm{O}\right) \delta 9.62(\mathrm{~s}, 1 \mathrm{H}), 8.43(\mathrm{~d}, J=6.7 \mathrm{~Hz}, 1 \mathrm{H}), 8.40-8.32(\mathrm{~m}, 2 \mathrm{H}), 8.25-8.13$ $(\mathrm{m}, 2 \mathrm{H}), 8.01(\mathrm{t}, J=7.4 \mathrm{~Hz}, 1 \mathrm{H})$.

${ }^{13} \mathrm{C}$ NMR (101 MHz, d $\left.\mathrm{d}_{2} \mathrm{O}\right) \delta 149.73,137.09,136.85,134.74,131.22,129.81,127.38,127.06,126.04$. HRMS (ESI): calculated for $\mathrm{C}_{10} \mathrm{H}_{7} \mathrm{D}_{3} \mathrm{~N}^{+} 147.0996$, found 147.0978.

\section{1-methylquinolin-1-ium iodide 5b}

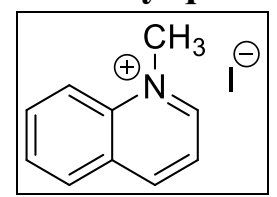

Synthesized according to the general procedure.

${ }^{1} \mathrm{H}$ NMR $\left(400 \mathrm{MHz}, \mathrm{DMSO}-d_{6}\right) \delta 9.55(\mathrm{~d}, J=5.2 \mathrm{~Hz}, 1 \mathrm{H}), 9.30(\mathrm{~d}, J=8.3 \mathrm{~Hz}, 1 \mathrm{H}), 8.56-8.45(\mathrm{~m}$, $2 \mathrm{H}), 8.29(\mathrm{t}, J=7.9 \mathrm{~Hz}, 1 \mathrm{H}), 8.19(\mathrm{t}, J=6.9 \mathrm{~Hz}, 1 \mathrm{H}), 8.07(\mathrm{t}, J=7.5 \mathrm{~Hz}, 1 \mathrm{H}), 4.65(\mathrm{~s}, 3 \mathrm{H})$.

${ }^{13} \mathrm{C}$ NMR $\left(101 \mathrm{MHz}, \mathrm{DMSO}-d_{6}\right) \delta 150.09,146.94,138.21,135.38,130.22,129.87,129.08,121.95$, 119.11, 45.43 .

HRMS (ESI): calculated for $\mathrm{C}_{10} \mathrm{H}_{10} \mathrm{~N}^{+}$144.0808, found 144.0766. 
1-(methyl-d3)quinolin-1-ium iodide 5c

${ }^{C^{\prime} D_{3}}$

Synthesized according to the general procedure.

${ }^{1} \mathrm{H}$ NMR (400 MHz, DMSO-d $\left.d_{6}\right) \delta .53(\mathrm{~d}, J=5.3 \mathrm{~Hz}, 1 \mathrm{H}), 9.30(\mathrm{~d}, J=8.3 \mathrm{~Hz}, 1 \mathrm{H}), 8.56-8.45$ (m, $2 \mathrm{H}), 8.29$ (t, $J=7.8 \mathrm{~Hz}, 1 \mathrm{H}), 8.19(\mathrm{~d}, J=7.0 \mathrm{~Hz}, 1 \mathrm{H}), 8.07$ (t, $J=7.5 \mathrm{~Hz}, 1 \mathrm{H})$.

${ }^{13} \mathrm{C}$ NMR $\left(101 \mathrm{MHz}, \mathrm{DMSO}-d_{6}\right) \delta 150.10,146.98,138.24,135.40,130.24,129.90,129.11,121.97$, 119.12 .

HRMS (ESI): calculated for $\mathrm{C}_{10} \mathrm{H}_{7} \mathrm{D}_{3} \mathrm{~N}^{+}$147.0996, found 147.0967.

\section{2-methyl-1,2,3,4-tetrahydroisoquinoline 6b}

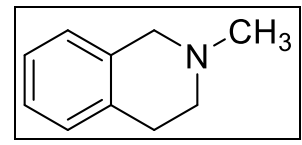

1,2,3,4-Tetrahydroisoquinoline 6 (533 $\mathrm{mg}, 1.0 \mathrm{eq}, 4 \mathrm{mmol})$ was dissolved in ethanol $(20 \mathrm{~mL})$ and potassium carbonate (1106 mg, 2.0 eq, $6 \mathrm{mmol}$ ) was added followed by methyl iodide ( $852 \mathrm{mg}, 1.5$ eq, $8 \mathrm{mmol}$ ). The mixture was stirred overnight at room temperature, concentrated, redissolved in water $(25 \mathrm{~mL})$ and extracted with $\mathrm{CHCl}_{3}(3 \times 25 \mathrm{~mL})$. The crude product was purified by column chromatography (5\% MeOH in DCM) yielding the product as a slightly brown oil (106 mg, 18\%) ${ }^{1} \mathrm{H}$ NMR $\left(400 \mathrm{MHz}, \mathrm{CDCl}_{3}\right) \delta 7.30-7.20(\mathrm{~m}, 2 \mathrm{H}), 7.18(\mathrm{~d}, J=7.4 \mathrm{~Hz}, 1 \mathrm{H}), 7.10(\mathrm{~d}, J=7.2 \mathrm{~Hz}, 1 \mathrm{H})$, $4.32(\mathrm{~s}, 2 \mathrm{H}), 3.48(\mathrm{t}, J=6.3 \mathrm{~Hz}, 2 \mathrm{H}), 3.28(\mathrm{t}, J=6.3 \mathrm{~Hz}, 2 \mathrm{H}), 2.92(\mathrm{~s}, 3 \mathrm{H})$.

${ }^{13} \mathrm{C}$ NMR $\left(101 \mathrm{MHz} \mathrm{CDCl}_{3}\right) \delta 130.21,129.04,128.63,127.53,126.81,126.57,54.53,51.40,42.77$, 24.94.

HRMS (ESI): calculated for $\mathrm{C}_{10} \mathrm{H}_{13} \mathrm{~N}(\mathrm{M}+\mathrm{H})^{+} 148.1121$, found 148.1160

\section{2-(methyl-d3)-1,2,3,4-tetrahydroisoquinoline 6c}

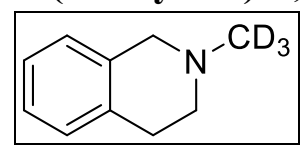

Prepared as described for compound $\mathbf{6 b}$ using $\mathrm{d}_{3}$-methyl iodide yielding a slightly brown oil (226 mg, $38 \%)$

${ }^{1} \mathrm{H}$ NMR (400 MHz, $\left.\mathrm{CDCl}_{3}\right) \delta 7.29-7.21(\mathrm{~m}, 2 \mathrm{H}), 7.20-7.14(\mathrm{~m}, 2 \mathrm{H}), 4.44(\mathrm{~s}, 2 \mathrm{H}), 3.55(\mathrm{t}, J=6.2$ $\mathrm{Hz}, 2 \mathrm{H}), 3.23(\mathrm{t}, J=6.0 \mathrm{~Hz}, 2 \mathrm{H})$.

${ }^{13} \mathrm{C}$ NMR (101 MHz, $\left.\mathrm{CDCl}_{3}\right) \delta 131.24,129.13,128.32,127.42,126.91,126.68,44.13,41.77,25.04$. HRMS (ESI): calculated for $\mathrm{C}_{10} \mathrm{H}_{11} \mathrm{D}_{3} \mathrm{~N}(\mathrm{M}+\mathrm{H})^{+} 151.1309$, found 151.1323

\section{3-carbamoyl-1-methylquinolin-1-ium iodide 7b}<smiles>CN1C(C(N)=O)=Cc2ccccc2N1C</smiles>

Synthesized according to the general procedure. 
${ }^{1} \mathrm{H}$ NMR (400 MHz, DMSO-d $\left.d_{6}\right) \delta 9.91(\mathrm{~s}, 1 \mathrm{H}), 9.69$ (s, 1H), 8.59 (br s, $\left.1 \mathrm{H}\right), 8.57(\mathrm{~d}, J=9.1 \mathrm{~Hz}, 1 \mathrm{H})$, $8.51(\mathrm{~d}, J=7.9 \mathrm{~Hz}, 1 \mathrm{H}), 8.37(\mathrm{t}, J=7.6 \mathrm{~Hz}, 1 \mathrm{H}), 8.17($ br s, $1 \mathrm{H}), 8.13(\mathrm{t}, J=7.6 \mathrm{~Hz}, 1 \mathrm{H}), 4.69$ (s, $3 \mathrm{H})$.

${ }^{13} \mathrm{C}$ NMR $\left(101 \mathrm{MHz}, \mathrm{DMSO}-d_{6}\right) \delta 163.27,149.95,145.11,138.74,136.78,131.33,130.58,128.14$, 127.52, 119.32, 45.65 .

HRMS (ESI): calculated for $\mathrm{C}_{11} \mathrm{H}_{11} \mathrm{~N}_{2} \mathrm{O}^{+} 187.0866$, found 187.0841 .

\section{3-carbamoyl-1-(methyl-d3)quinolin-1-ium iodide 7c}

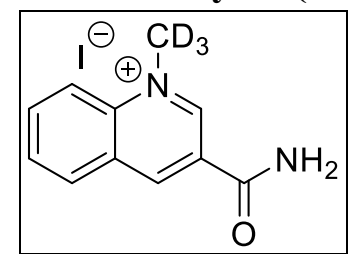

Synthesized according to the general procedure.

${ }^{1} \mathrm{H}$ NMR (400 MHz, DMSO-d $\left.d_{6}\right) \delta 9.90(\mathrm{~s}, 1 \mathrm{H}), 9.68(\mathrm{~s}, 1 \mathrm{H}), 8.59(\mathrm{br} \mathrm{s}, 1 \mathrm{H}), 8.56(\mathrm{~d}, J=9.0 \mathrm{~Hz}, 1 \mathrm{H})$, $8.51(\mathrm{~d}, J=8.2 \mathrm{~Hz}, 1 \mathrm{H}), 8.37$ (t, $J=7.9 \mathrm{~Hz}, 1 \mathrm{H}), 8.17$ (br s, 1H), 8.13 (t, $J=7.6 \mathrm{~Hz}, 1 \mathrm{H})$.

${ }^{13} \mathrm{C}$ NMR $\left(101 \mathrm{MHz}, \mathrm{DMSO}-d_{6}\right) \delta 163.28,149.92,145.12,138.73,136.77,131.32,130.58,128.13$, 127.50, 119.32 .

HRMS (ESI): calculated for $\mathrm{C}_{11} \mathrm{H}_{8} \mathrm{D}_{3} \mathrm{~N}_{2} \mathrm{O}^{+} 190.1054$, found 190.1030 .

\section{3-acetyl-1-methylpyridin-1-ium iodide 8b}<smiles></smiles>

Synthesized according to the general procedure.

${ }^{1} \mathrm{H}$ NMR (400 MHz, d 20$) \delta 9.43(\mathrm{~s}, 1 \mathrm{H}), 9.16-8.92(\mathrm{~m}, 2 \mathrm{H}), 8.25(\mathrm{t}, J=6.9 \mathrm{~Hz}, 1 \mathrm{H}), 4.53(\mathrm{~s}, 3 \mathrm{H})$, $2.82(\mathrm{~s}, 3 \mathrm{H})$.

${ }^{13} \mathrm{C}$ NMR $\left(101 \mathrm{MHz}, \mathrm{d}_{2} \mathrm{O}\right) \delta 196.43,147.91,145.81,144.24,135.51,128.23,48.63,26.61$. HRMS (ESI): calculated for $\mathrm{C}_{8} \mathrm{H}_{10} \mathrm{NO}^{+} 136.0757$, found 136.0731 .

\section{3-acetyl-1-(methyl-d3)pyridin-1-ium iodide 8c}

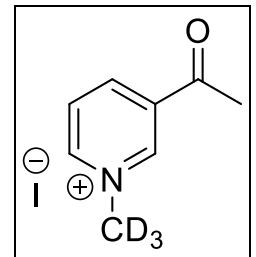

Synthesized according to the general procedure.

${ }^{1} \mathrm{H}$ NMR (400 MHz, d 20$) \delta 9.43(\mathrm{~s}, 1 \mathrm{H}), 9.14-8.93(\mathrm{~m}, 2 \mathrm{H}), 8.26(\mathrm{t}, J=7.1 \mathrm{~Hz}, 1 \mathrm{H}), 2.82(\mathrm{~s}, 3 \mathrm{H})$.

${ }^{13} \mathrm{C}$ NMR (101 MHz, d $\left.\mathrm{d}_{2} \mathrm{O}\right) \delta 196.44,147.89,145.80,144.26,135.51,128.24,26.63$.

HRMS (ESI): calculated for $\mathrm{C}_{8} \mathrm{H}_{7} \mathrm{D}_{3} \mathrm{NO}^{+} 139.0945$, found 139.0914 . 
$\overbrace{\substack{\mathrm{CH}_{3} \\ \mathrm{CH}_{2}}}^{\mathrm{NH}}$

Synthesized according to the general procedure.

${ }^{1} \mathrm{H}$ NMR $\left(400 \mathrm{MHz}, \mathrm{DMSO}-d_{6}\right) \delta 9.85(\mathrm{br} \mathrm{s}, 3 \mathrm{H}), 9.65(\mathrm{~s}, 1 \mathrm{H}), 9.25(\mathrm{~d}, J=6.1 \mathrm{~Hz}, 1 \mathrm{H}), 8.93(\mathrm{~d}, J=$ $8.2 \mathrm{~Hz}, 1 \mathrm{H}), 8.36(\mathrm{dd}, J=7.9,6.4 \mathrm{~Hz}, 1 \mathrm{H})$.

${ }^{13} \mathrm{C}$ NMR (101 MHz, DMSO- $\left.d_{6}\right) \delta 160.90,148.90,146.11,144.77,127.50,127.17,48.40$.

HRMS (ESI): calculated for $\mathrm{C}_{7} \mathrm{H}_{10} \mathrm{~N}_{3}{ }^{+} 136.0869$, found 136.0814 .

3-carbamimidoyl-1-(methyl-d3)pyridin-1-ium iodide 9c

$\overbrace{\substack{C_{1} \\ C_{2}}}^{N}$

Synthesized according to the general procedure.

${ }^{1} \mathrm{H}$ NMR (400 MHz, DMSO-d $) \delta 9.83$ (br s, 3H), $9.63(\mathrm{~s}, 1 \mathrm{H}), 9.25$ (d, $\left.J=6.1 \mathrm{~Hz}, 1 \mathrm{H}\right), 8.93(\mathrm{~d}, J=$ $8.3 \mathrm{~Hz}, 1 \mathrm{H}), 8.36(\mathrm{dd}, J=8.0,6.3 \mathrm{~Hz}, 1 \mathrm{H})$.

${ }^{13} \mathrm{C}$ NMR (101 MHz, DMSO- $\left.d_{6}\right) \delta 160.90,148.90,146.09,144.79,127.54,127.18$.

HRMS (ESI): calculated for $\mathrm{C}_{7} \mathrm{H}_{7} \mathrm{D}_{3} \mathrm{~N}_{3}{ }^{+} 139.1058$, found 139.1012 .

\section{1,2-dimethylpyridin-1-ium iodide $10 \mathrm{~b}$}

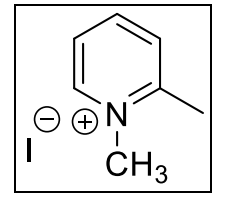

Synthesized according to the general procedure.

${ }^{1} \mathrm{H}$ NMR (400 MHz, DMSO- $\left.d_{6}\right) \delta 8.98(\mathrm{~d}, J=6.1 \mathrm{~Hz}, 1 \mathrm{H}), 8.47(\mathrm{t}, J=7.8 \mathrm{~Hz}, 1 \mathrm{H}), 8.05(\mathrm{~d}, J=7.9$ $\mathrm{Hz}, 1 \mathrm{H}), 7.94$ (t, $J=6.8 \mathrm{~Hz}, 1 \mathrm{H}), 4.24$ (s, 3H), 2.79 (s, 3H).

${ }^{13} \mathrm{C}$ NMR (101 MHz, DMSO-d $\left.d_{6}\right) \delta 155.84,145.97,144.93,129.05,125.15,45.53,20.02$.

HRMS (ESI): calculated for $\mathrm{C}_{7} \mathrm{H}_{10} \mathrm{~N}^{+} 108.0808$, found 108.0820 .

\section{2-methyl-1-(methyl-d3)pyridin-1-ium iodide 10c}

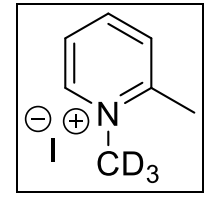

Synthesized according to the general procedure.

${ }^{1} \mathrm{H}$ NMR (400 MHz, DMSO-d $)_{6} \delta 8.97(\mathrm{~d}, J=5.8 \mathrm{~Hz}, 1 \mathrm{H}), 8.47(\mathrm{t}, J=7.5 \mathrm{~Hz}, 1 \mathrm{H}), 8.04(\mathrm{~d}, J=7.8$ $\mathrm{Hz}, 1 \mathrm{H}), 7.94(\mathrm{t}, J=6.4 \mathrm{~Hz}, 1 \mathrm{H}), 2.78(\mathrm{~s}, 3 \mathrm{H})$.

${ }^{13} \mathrm{C}$ NMR (101 MHz, DMSO- $\left.d_{6}\right) \delta 155.85,145.97,144.95,129.04,125.14,19.91$. HRMS (ESI): calculated for $\mathrm{C}_{7} \mathrm{H}_{7} \mathrm{D}_{3} \mathrm{~N}^{+} 111.0996$, found 111.1017 . 


\section{2-methoxy-1-methylpyridin-1-ium iodide 11b}

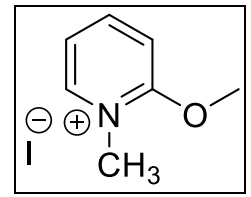

To a cold $\left(0^{\circ} \mathrm{C}\right)$ solution of 2-methoxypyridine $11(445 \mathrm{mg}, 4 \mathrm{mmol})$ in toluene $(4 \mathrm{~mL})$ was added methyl trifluoromethanesulfonate $(464 \mu \mathrm{L}(673 \mathrm{mg}), 4.1 \mathrm{mmol})$. The mixture was allowed to warm to room temperature, which resulted in the formation of a white crystalline precipitate. After 1 hour, the white solid was collected by filtration of the crude reaction mixture through a fritted glass funnel, followed by drying under vacuum yielding $984 \mathrm{mg}(91 \%)$ of the product as the triflate salt.

${ }^{1} \mathrm{H}$ NMR (400 MHz, d $\left.\mathrm{d}_{2} \mathrm{O}\right) \delta 8.54-8.32(\mathrm{~m}, 2 \mathrm{H}), 7.59$ (d, $\left.J=6.9 \mathrm{~Hz}, 1 \mathrm{H}\right), 7.54-7.40(\mathrm{br} \mathrm{m}, 1 \mathrm{H}), 4.31$ (s, $3 \mathrm{H}), 4.06(\mathrm{~s}, 3 \mathrm{H})$

${ }^{13} \mathrm{C}$ NMR $\left(101 \mathrm{MHz}, \mathrm{d}_{2} \mathrm{O}\right) \delta 160.65,147.74,143.05,121.13$ (triflate), 118.30, 117.98 (triflate), $110.59,58.70,41.14$.

HRMS (ESI): calculated for $\mathrm{C}_{7} \mathrm{H}_{10} \mathrm{NO}^{+} 124.0757$, found 124.0721 .

\section{2-methoxy-1-(methyl-d3)pyridin-1-ium iodide 11c}

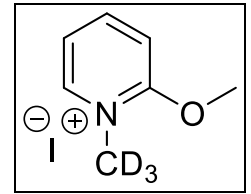

Deuterated methyl triflate was freshly prepared by mixing silver triflate (900 $\mathrm{mg}, 3.5 \mathrm{mmol}$ ) and methyl-d3-iodide $(507 \mathrm{mg}, 3.5 \mathrm{mmol})$ in $\mathrm{CCl}_{4}(6 \mathrm{~mL})$ at room temperature overnight. After filtration over cotton and flushing with $1 \mathrm{~mL} \mathrm{CCl} 4,7 \mathrm{~mL}$ of a $0.5 \mathrm{M} \mathrm{CD}_{3} \mathrm{OTf}$ solution was obtained and used as is.

2-methoxypyridine $11(110 \mathrm{mg}, 1 \mathrm{mmol})$ in $\mathrm{CCl}_{4}(0.5 \mathrm{~mL})$ was added to $2.2 \mathrm{~mL}$ of the $\mathrm{CD}_{3} \mathrm{OTf}$ solution at $0^{\circ} \mathrm{C}$ and the mixture was stirred for 1.5 hours at room temperature. The product was filtered off and flushed with dry diethyl ether yielding $140 \mathrm{mg}(51 \%)$ white product as the triflate salt.

${ }^{1} \mathrm{H}$ NMR $\left(400 \mathrm{MHz}, \mathrm{d}_{2} \mathrm{o}\right) \delta 8.44(\mathrm{t}, J=7.9 \mathrm{~Hz}, 1 \mathrm{H}), 8.39(\mathrm{~d}, J=6.3 \mathrm{~Hz}, 1 \mathrm{H}), 7.59(\mathrm{~d}, J=8.9 \mathrm{~Hz}$, $1 \mathrm{H}), 7.48(\mathrm{t}, J=6.8 \mathrm{~Hz}, 1 \mathrm{H}), 4.30(\mathrm{~s}, 3 \mathrm{H})$.

${ }^{13} \mathrm{C}$ NMR $\left(101 \mathrm{MHz}, \mathrm{d}_{2} \mathrm{O}\right) \delta 160.65,147.75,143.01,121.12$ (triflate), 118.29, 117.97 (triflate), 110.57, 58.68.

HRMS (ESI): calculated for $\mathrm{C}_{7} \mathrm{H}_{7} \mathrm{D}_{3} \mathrm{NO}^{+}$127.0945, found 127.0911 . 
Curves of the Michaelis-Menten and substrate inhibition kinetics for compounds 1-11

Nicotinamide (Compound 1)

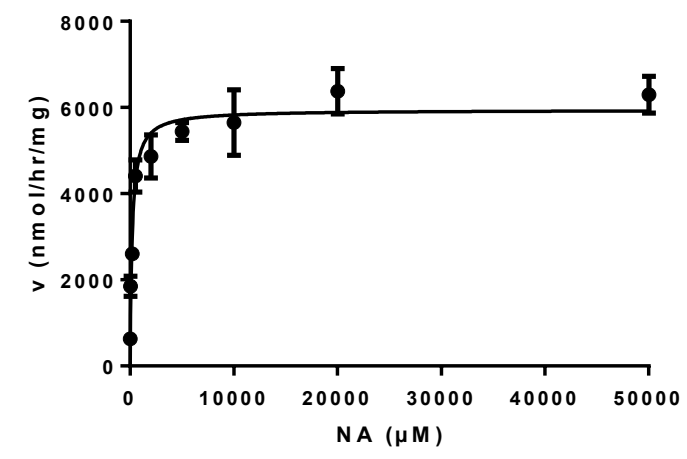

Thionicotinamide (Compound 2)

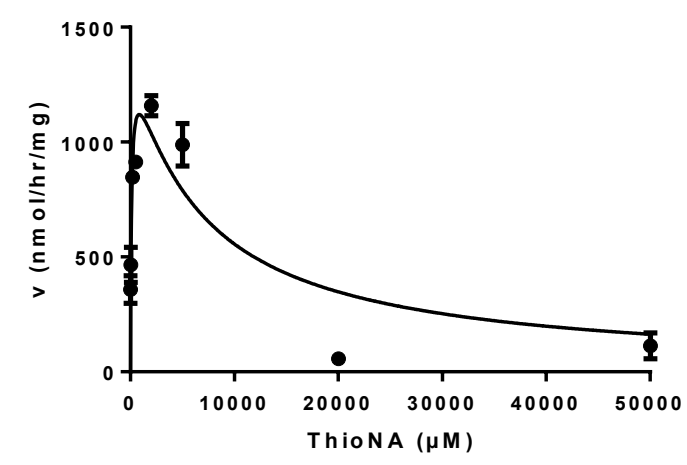

4-Methylnicotinamide (Compound 3)

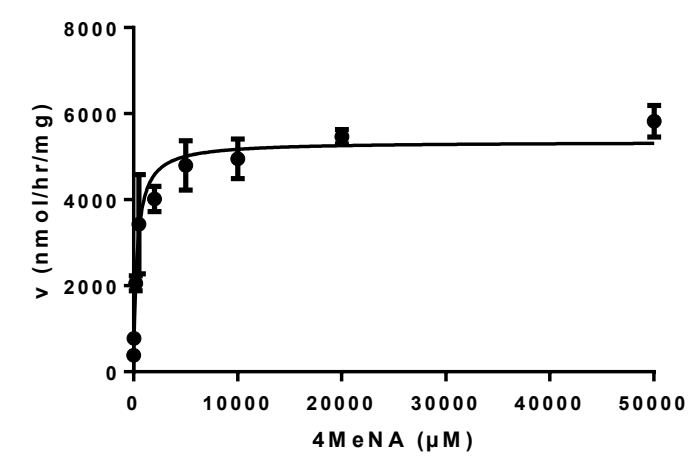

Isoquinoline (Compound 4)

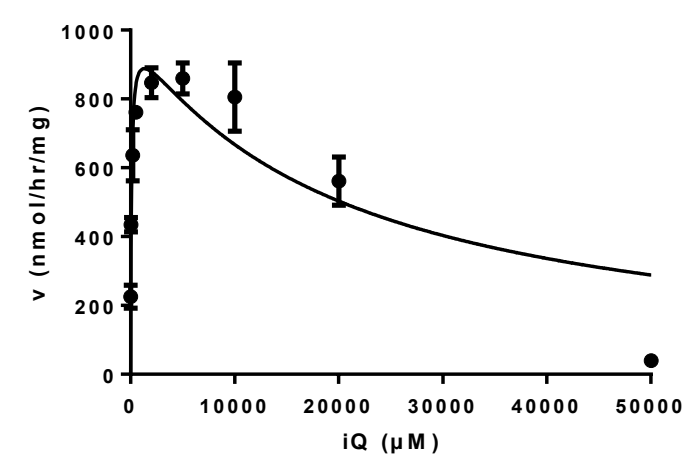


Quinoline (Compound 5)

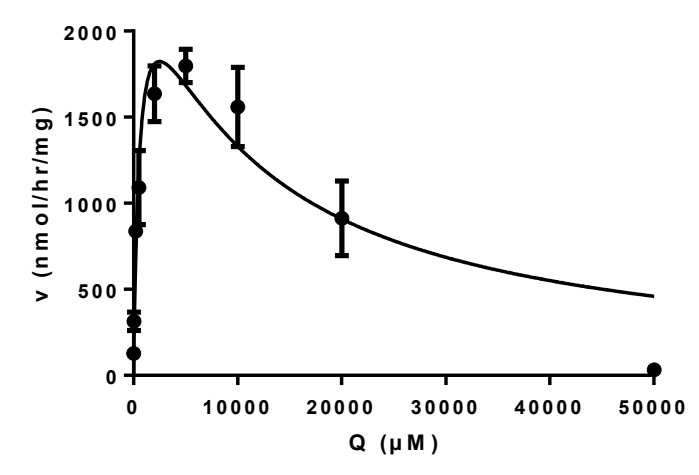

1,2,3,4-Tetrahydroisoquinoline (Compound 6) ${ }^{\mathrm{a}}$

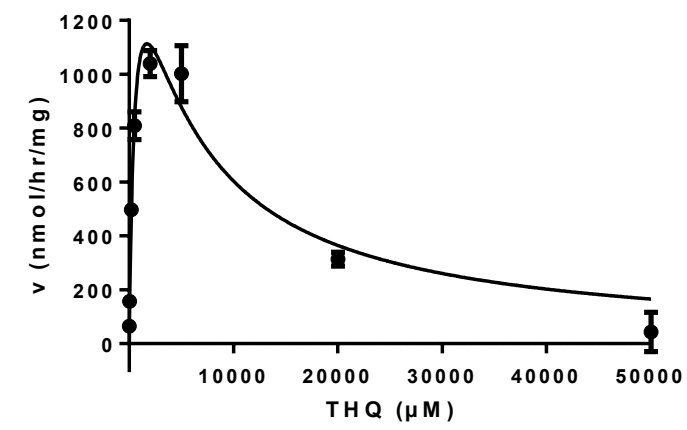

${ }^{\text {a }}$ Data corrected for an impurity in the substrate (about $0.005 \%$ ) that exhibited the same retention time and $m / z$ as the measured product.

Quinoline-3-carboxamide (Compound 7)

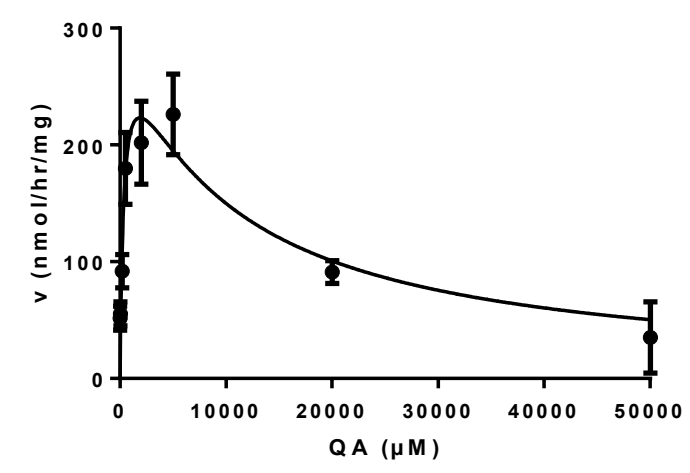

3-Acetylpyridine (Compound 8)

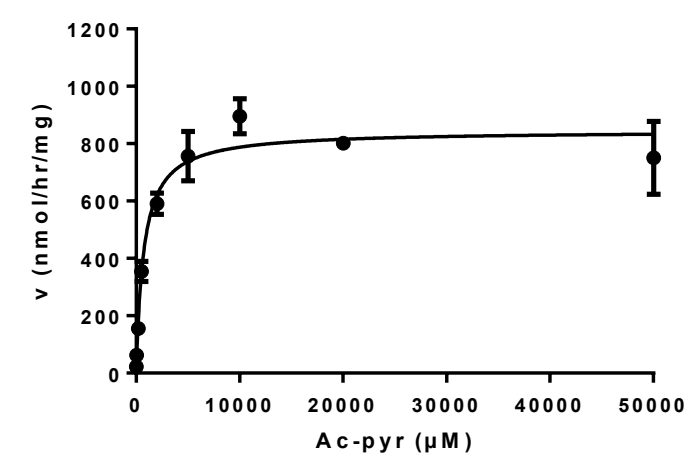


Nicotinimidamide (Compound 9)

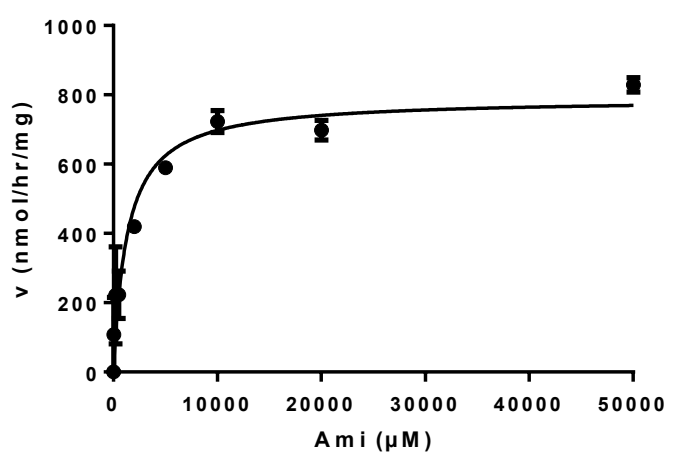

2-Methylpyridine (Compound 10)

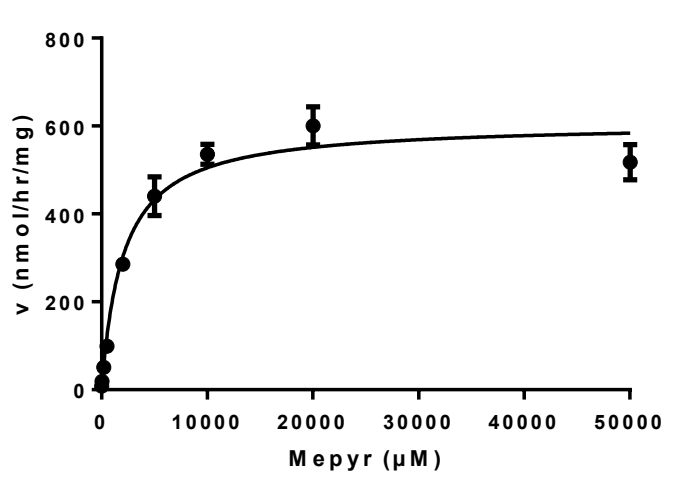

2-Methoxypyridine (Compound 11)

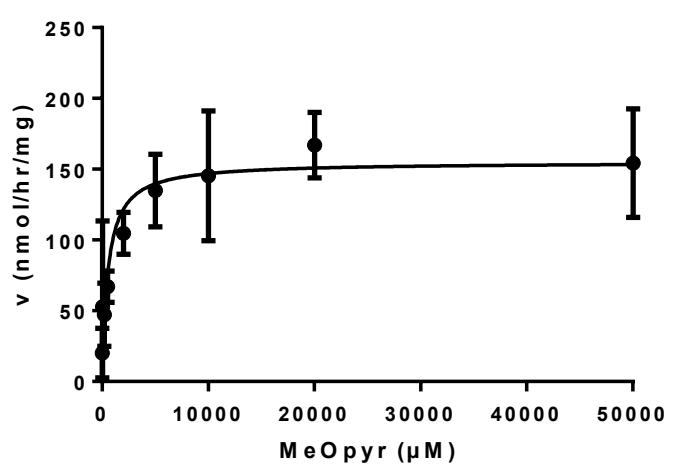


${ }^{1} \mathrm{H}$ and ${ }^{13} \mathrm{C}$ NMR spectra for compounds 1-11

Compound 1c: ${ }^{1}$ H NMR (400 MHz, DMSO-d $)$

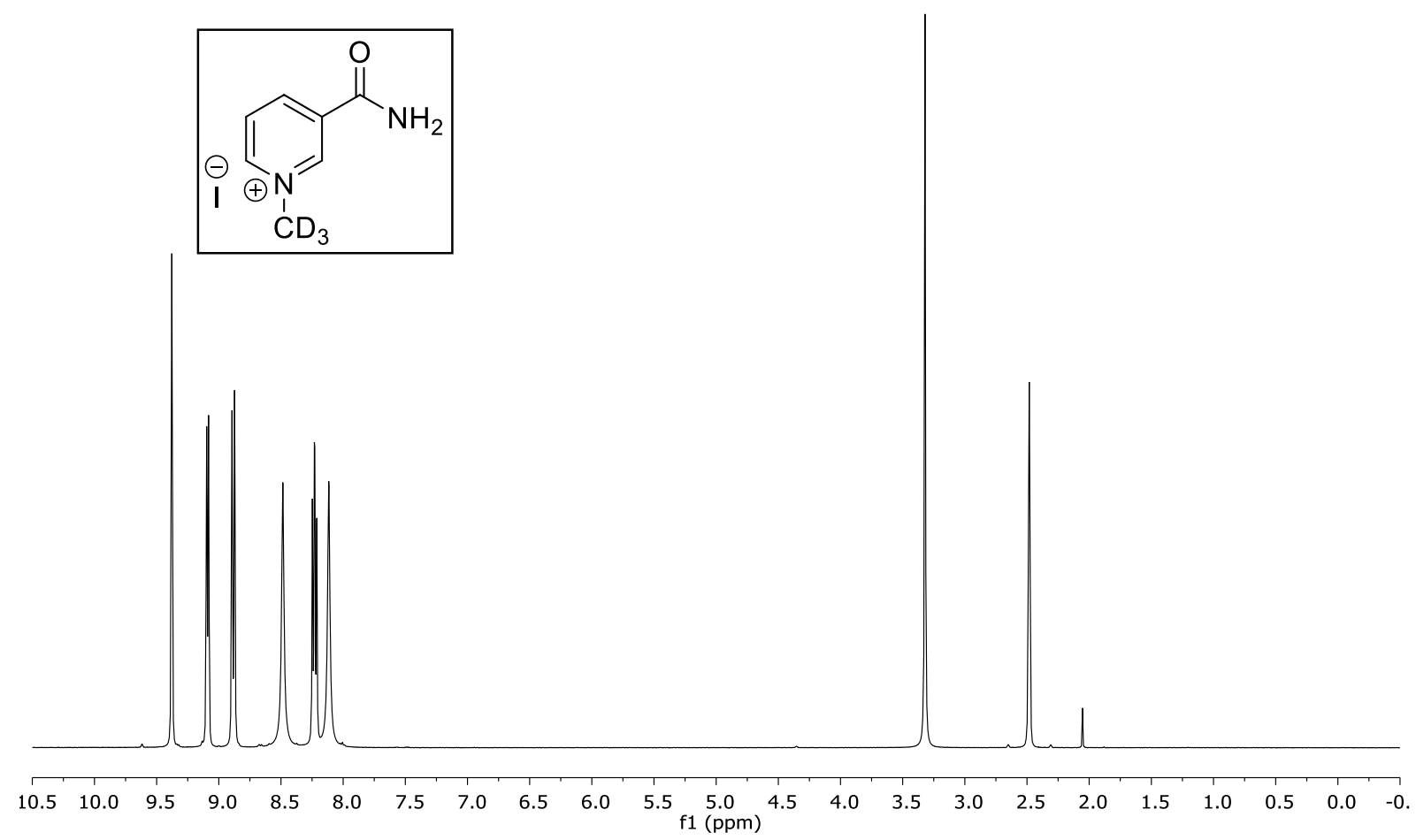

Compound 1c: ${ }^{13} \mathrm{C}$ NMR (101 MHz, DMSO-d 6$)$
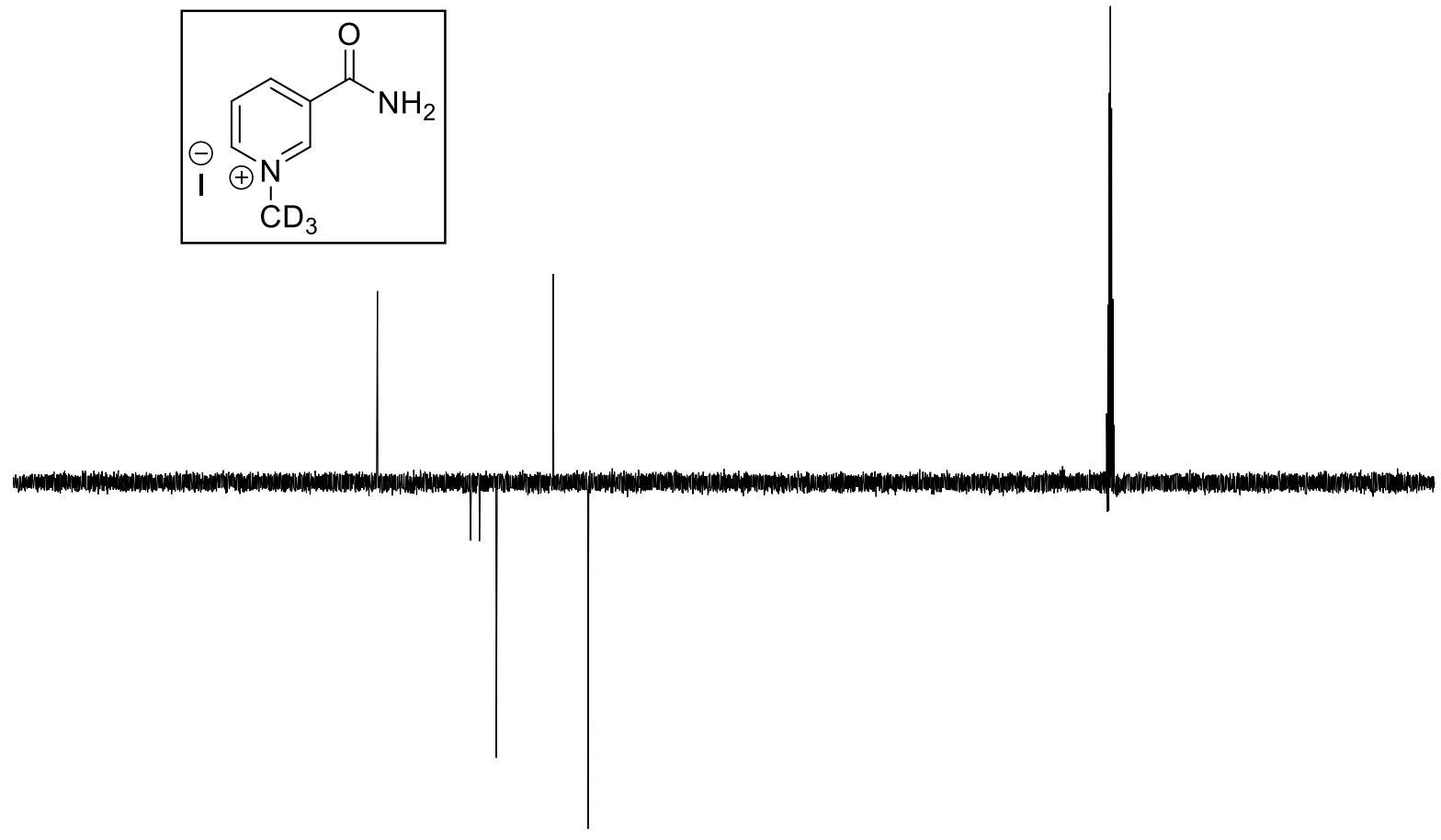

$\begin{array}{llllllllllllllllllllllll}220 & 210 & 200 & 190 & 180 & 170 & 160 & 150 & 140 & 130 & 120 & \begin{array}{c}110 \\ \mathrm{f} 1(\mathrm{ppm})\end{array} & 90 & 80 & 70 & 60 & 50 & 40 & 30 & 20 & 10 & 0 & -10\end{array}$ 
3-cyano-1-methylpyridin-1-ium iodide: ${ }^{1} \mathrm{H}$ NMR (400 MHz, DMSO-d 6 )
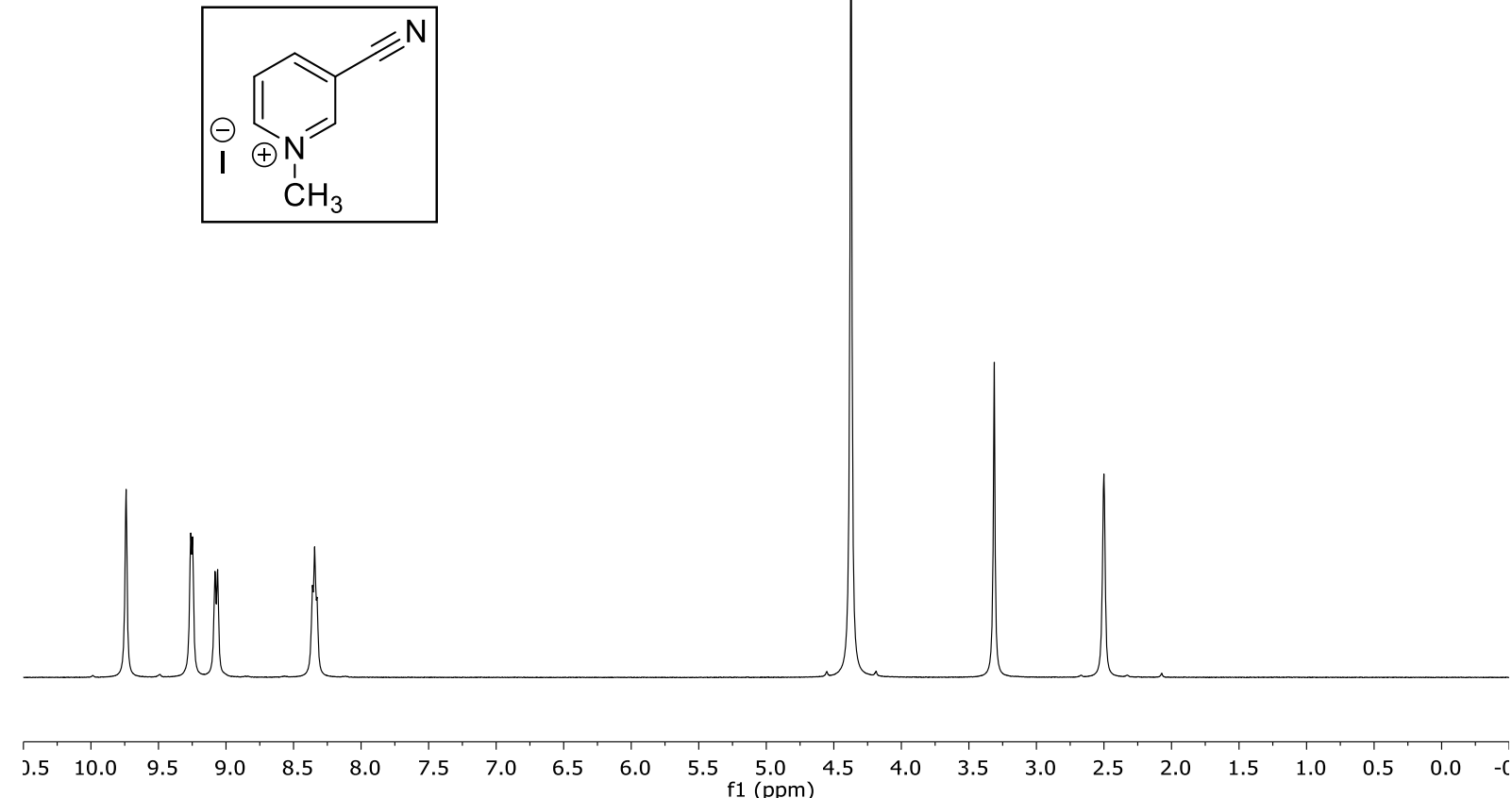

3-cyano-1-methylpyridin-1-ium iodide: ${ }^{13} \mathrm{C}$ NMR (101 MHz, DMSO-d $\left.{ }_{6}\right)$
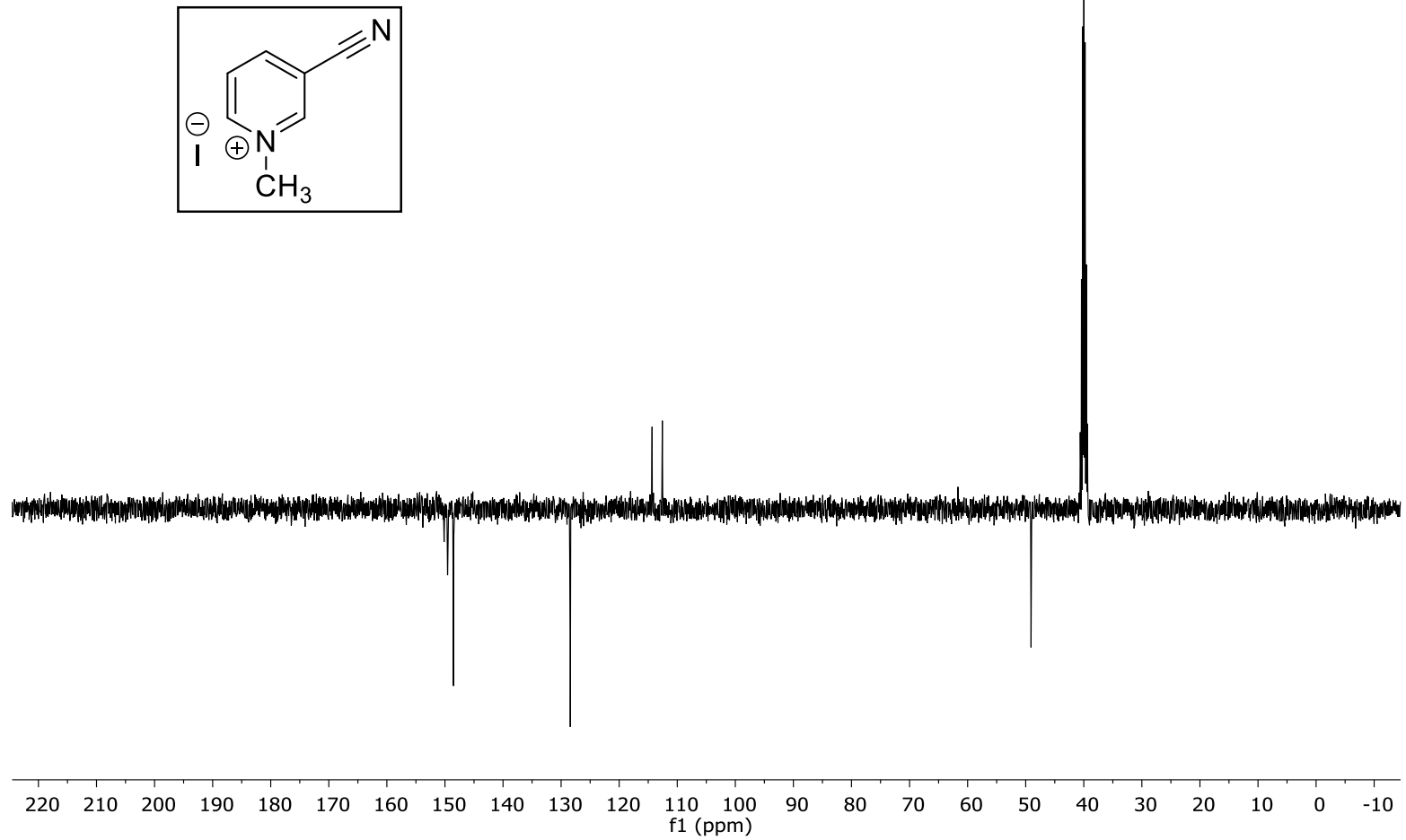

3-cyano-1-(methyl-d3)pyridin-1-ium iodide 
3-cyano-1-(methyl-d3)pyridin-1-ium iodide: ${ }^{1}$ H NMR (400 MHz, DMSO-d 6 )

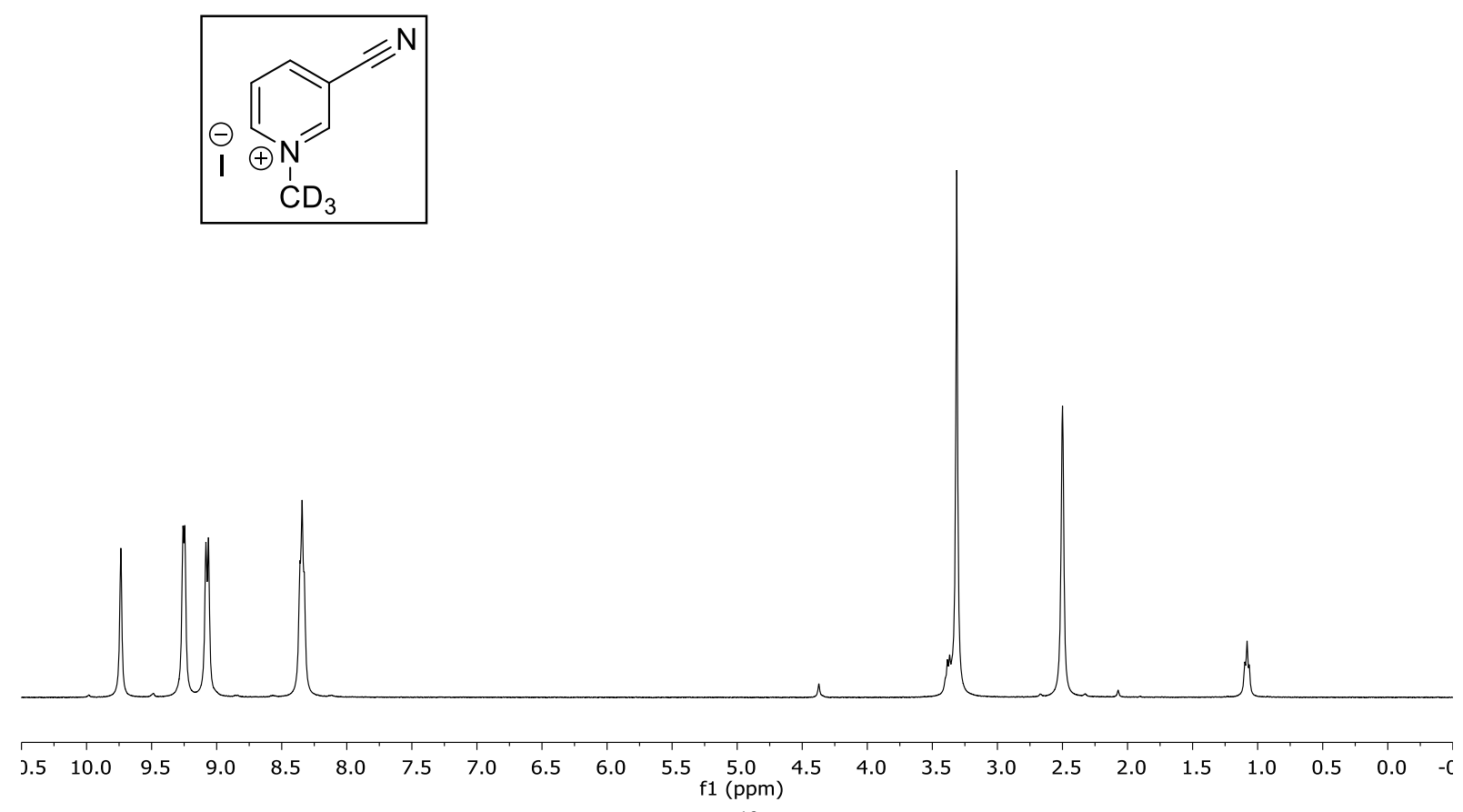

3-cyano-1-(methyl-d3)pyridin-1-ium iodide: ${ }^{13} \mathrm{C}$ NMR (101 MHz, DMSO-d 6 )
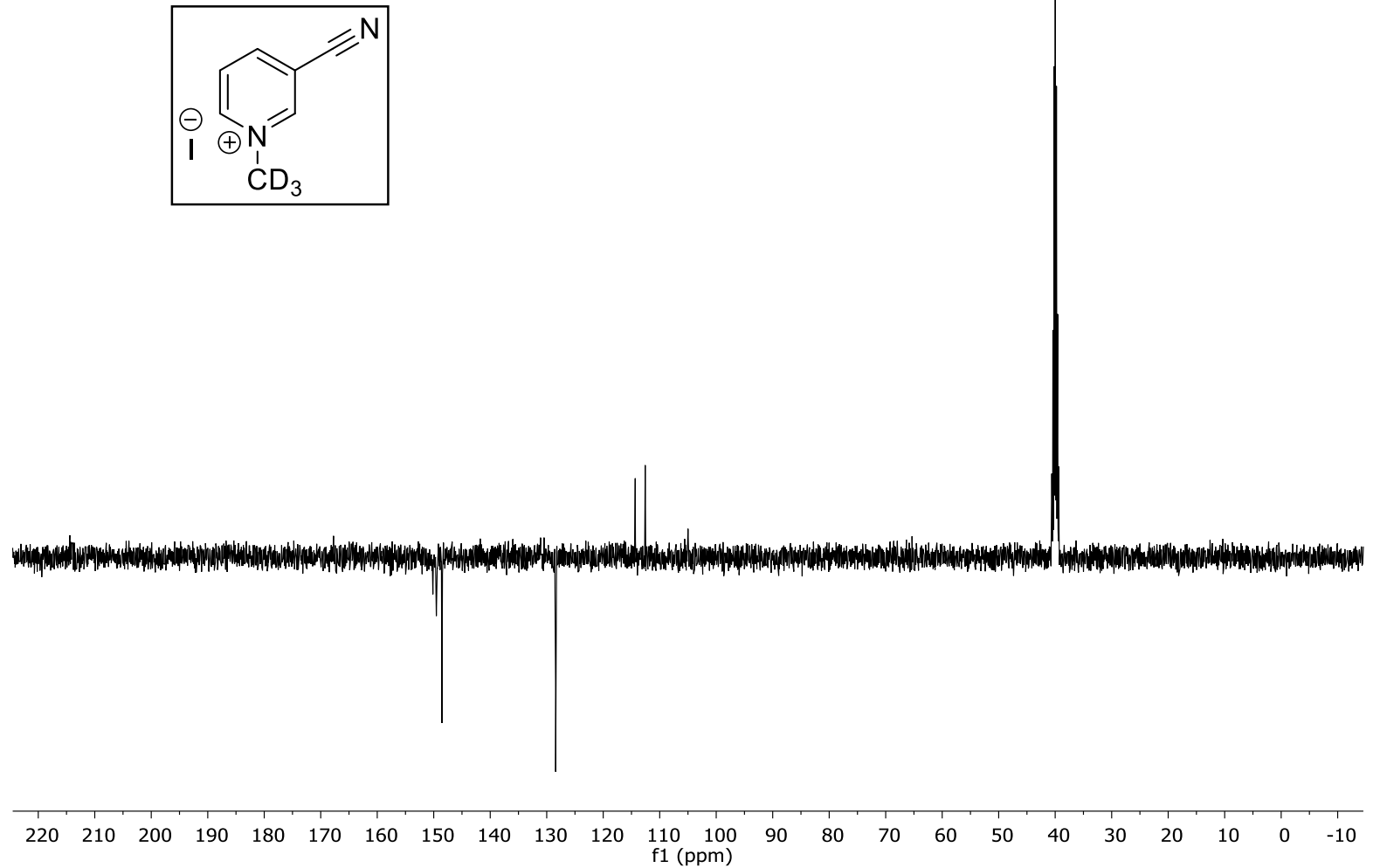
Compound 2b: ${ }^{1}$ H NMR (400 MHz, DMSO-d 6$)$
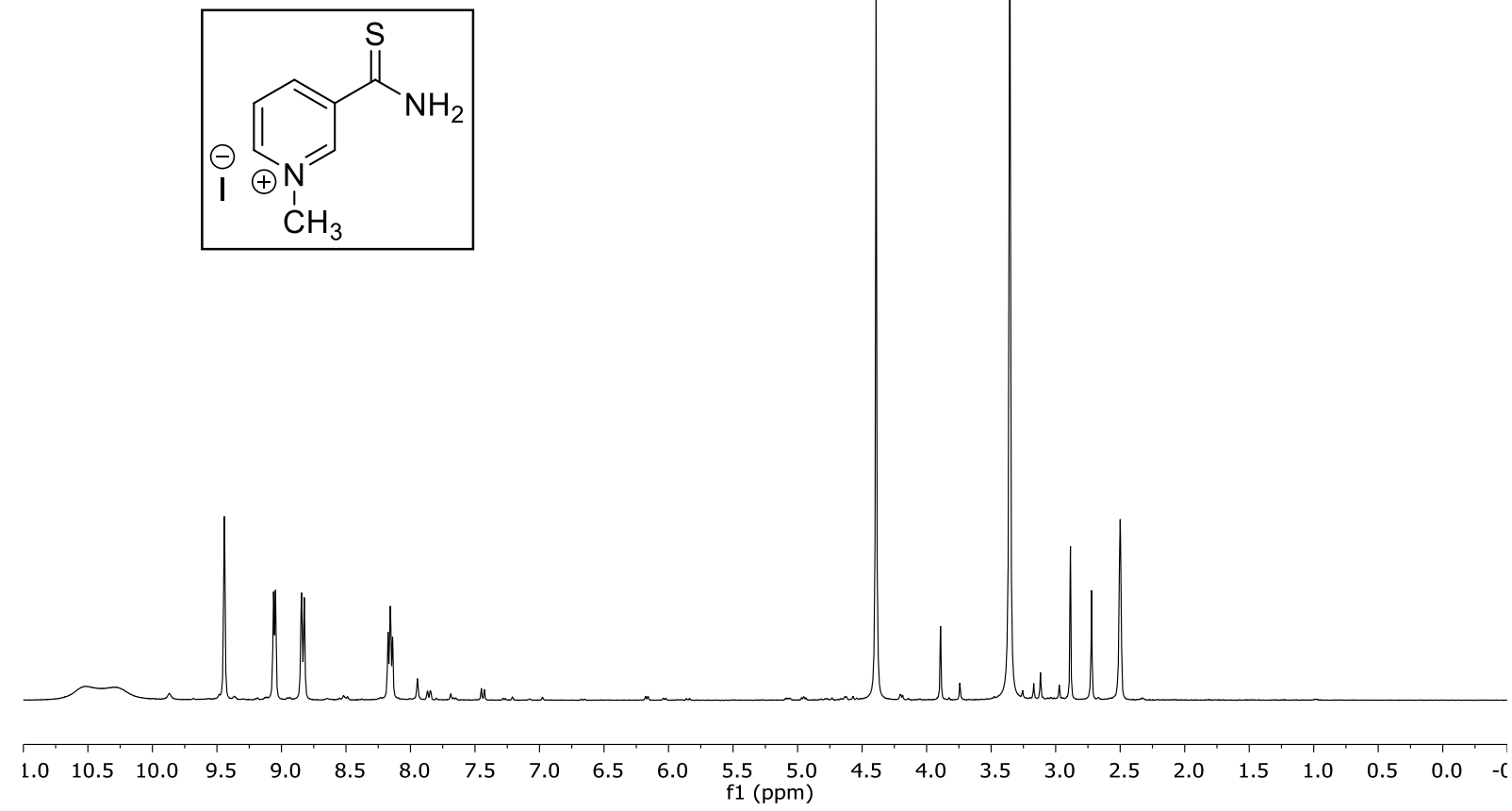

Compound 2b: ${ }^{13} \mathrm{C}$ NMR (101 MHz, DMSO-d 6 )
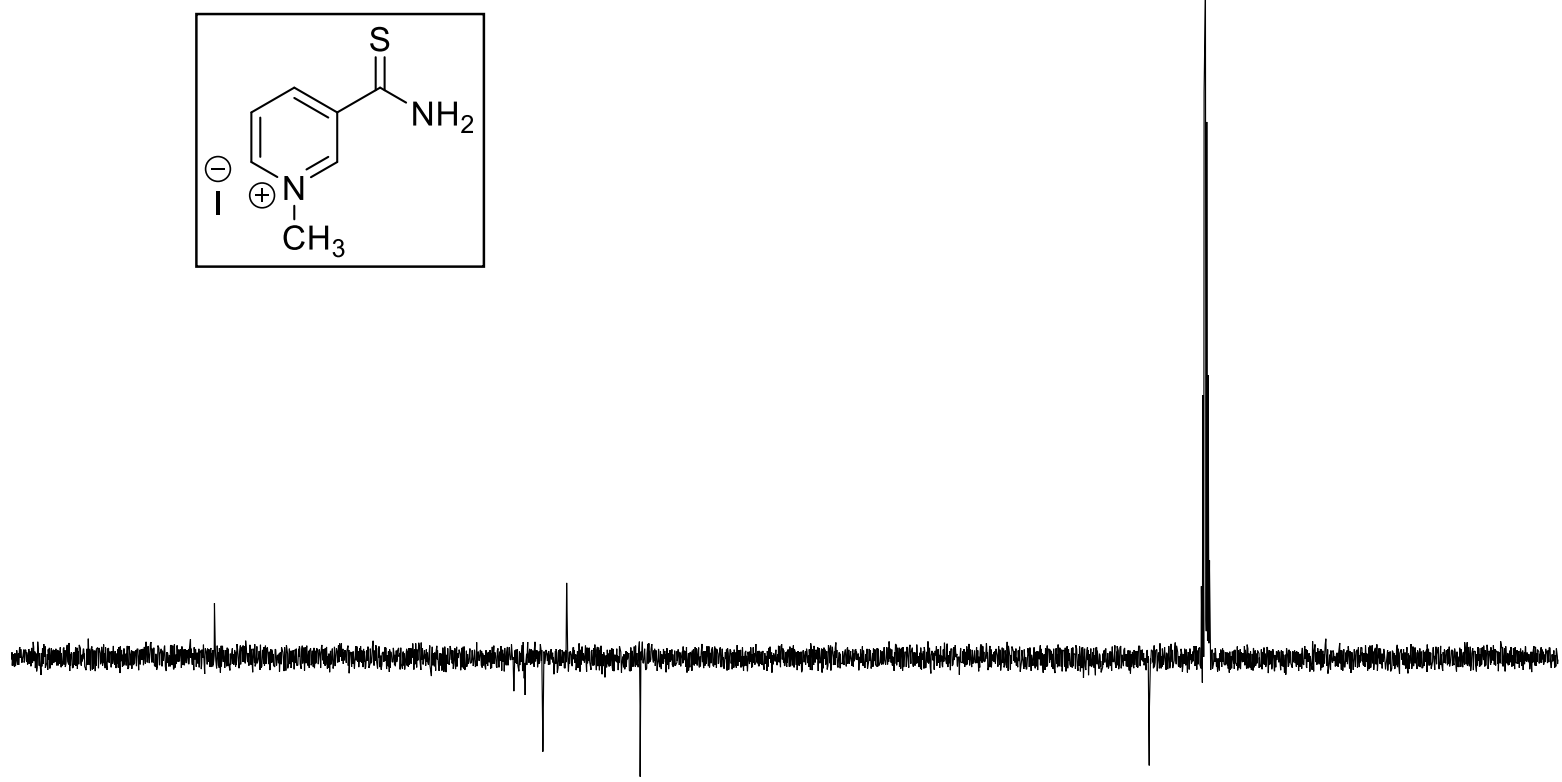

$\begin{array}{lllllllllllllllllllllll}220 & 210 & 200 & 190 & 180 & 170 & 160 & 150 & 140 & 130 & 120 & \underset{f 1}{110}(\mathrm{ppm}) & 90 & 80 & 70 & 60 & 50 & 40 & 30 & 20 & 10 & 0 & -10\end{array}$ 
Compound 2c: ${ }^{1} \mathrm{H}$ NMR (400 MHz, DMSO-d $)$
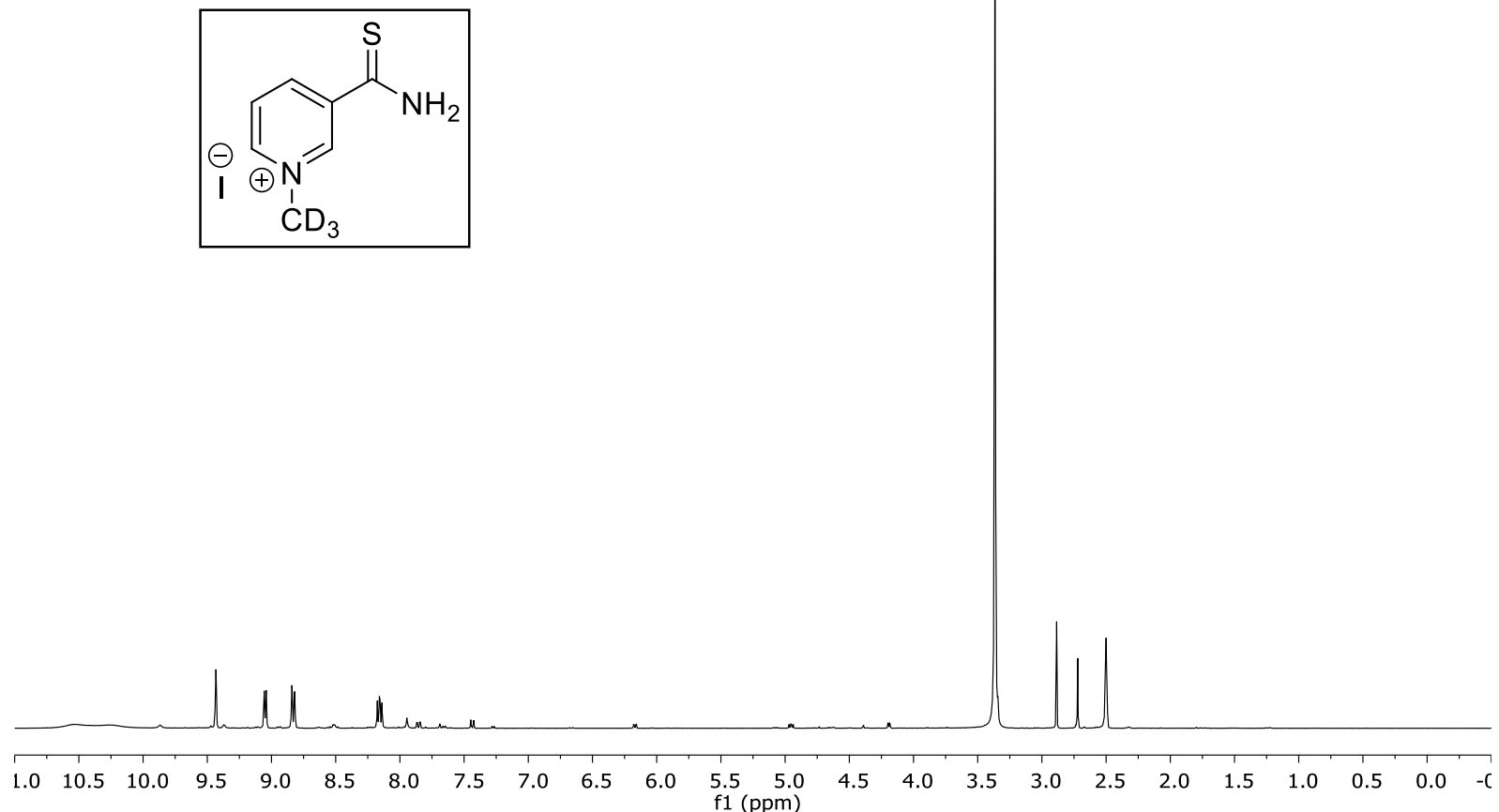

Compound 2c: ${ }^{13}$ C NMR (101 MHz, DMSO-d 6 )

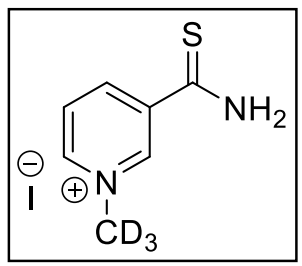

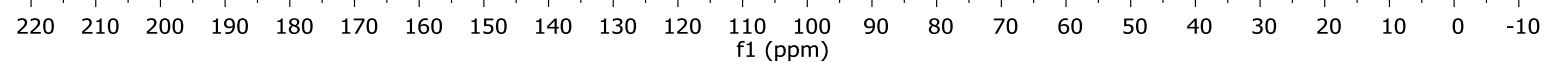




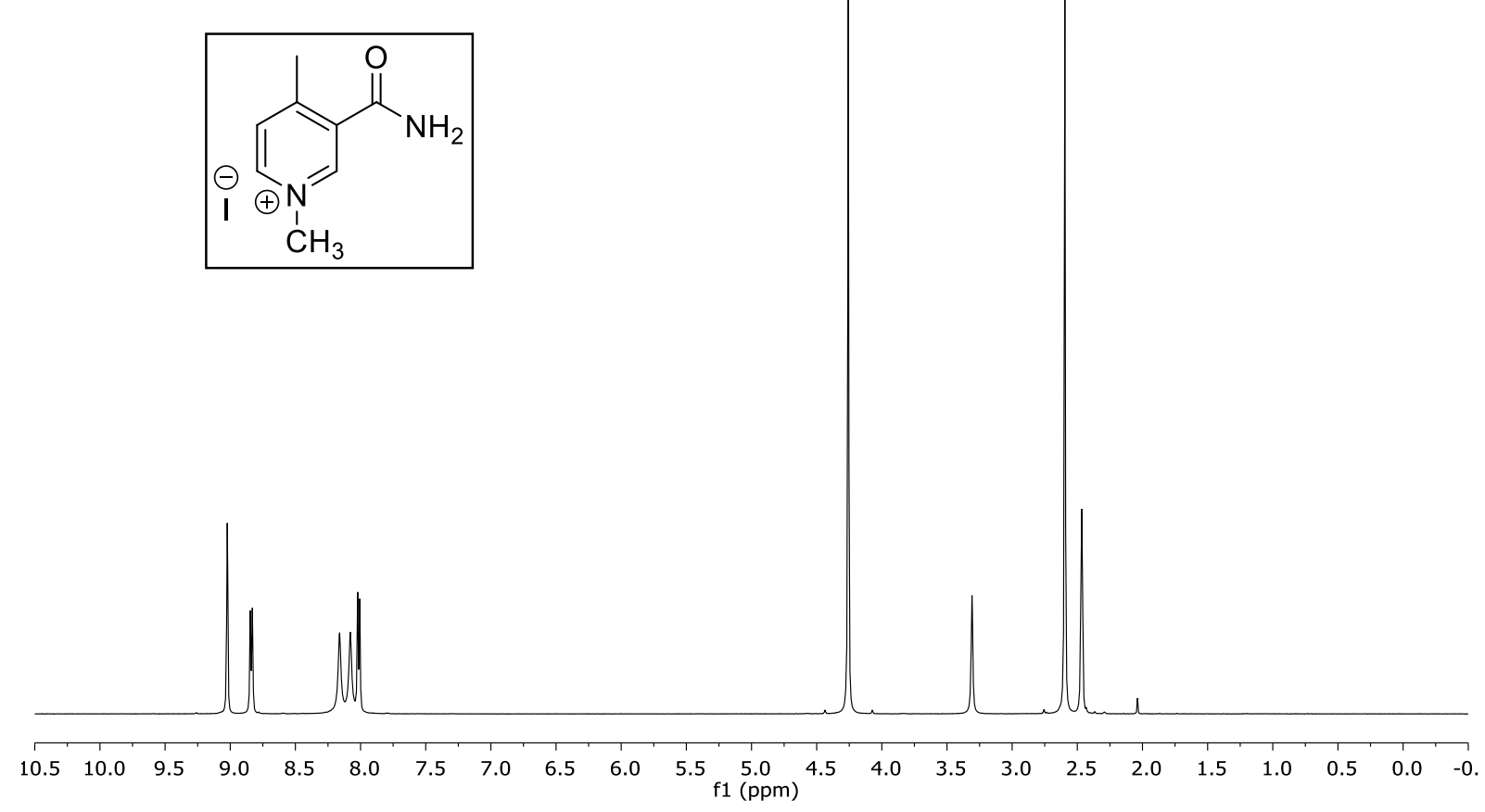

Compound 3b: ${ }^{13} \mathrm{C}$ NMR (101 MHz, DMSO-d 6 )
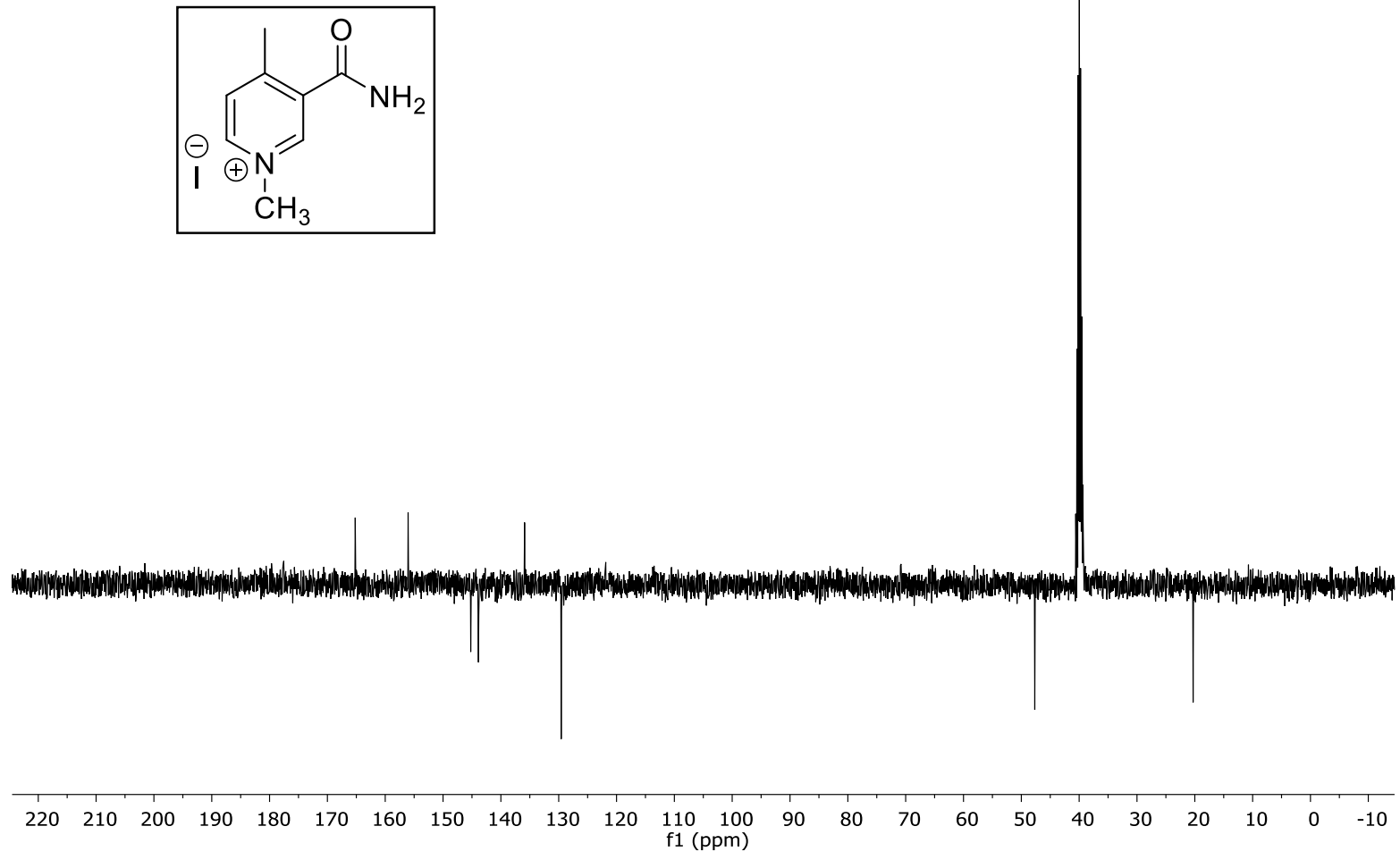
Compound 3c: ${ }^{1}$ H NMR (400 MHz, DMSO-d 6$)$
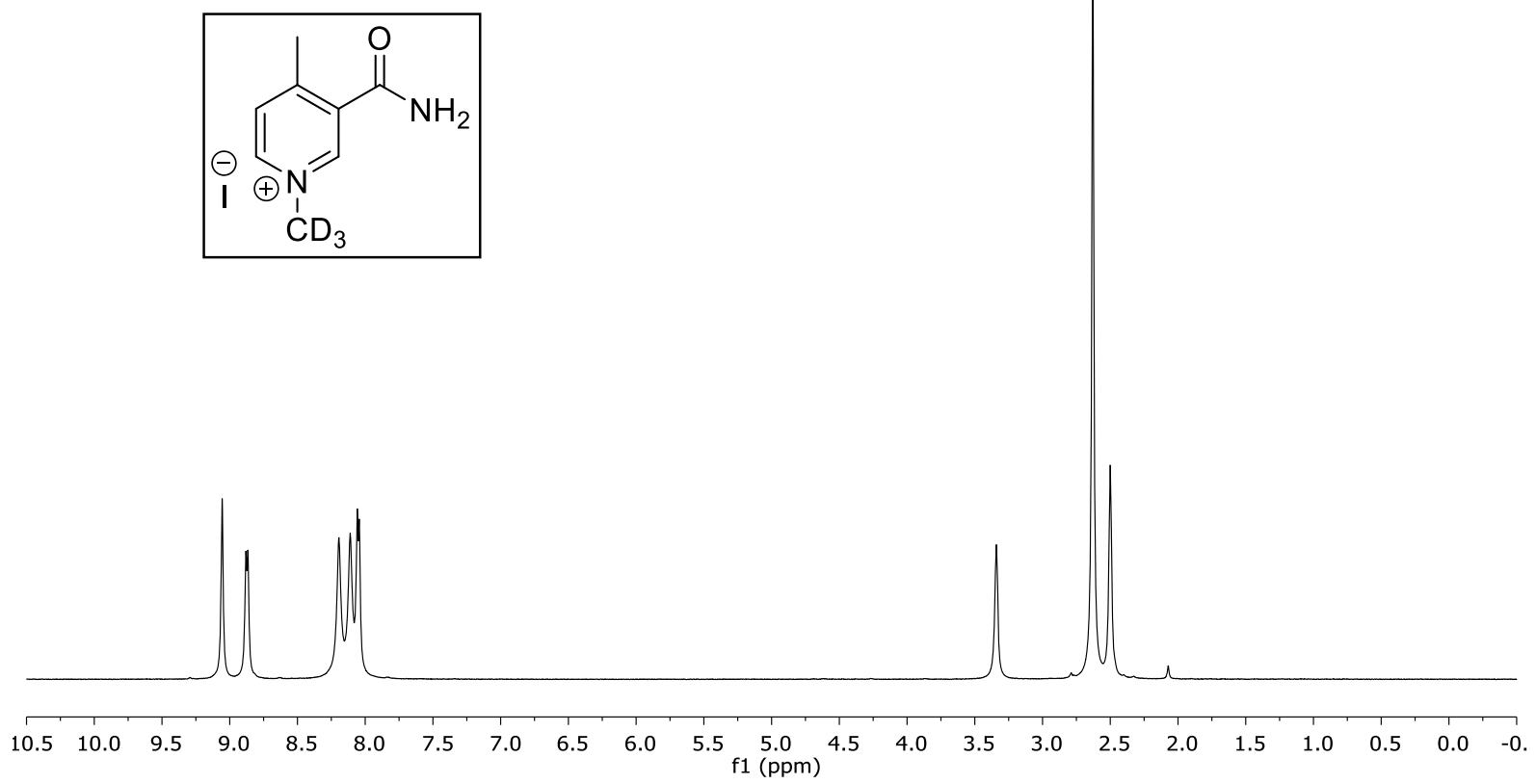

Compound 3c: ${ }^{13} \mathrm{C}$ NMR (101 MHz, DMSO-d 6$)$
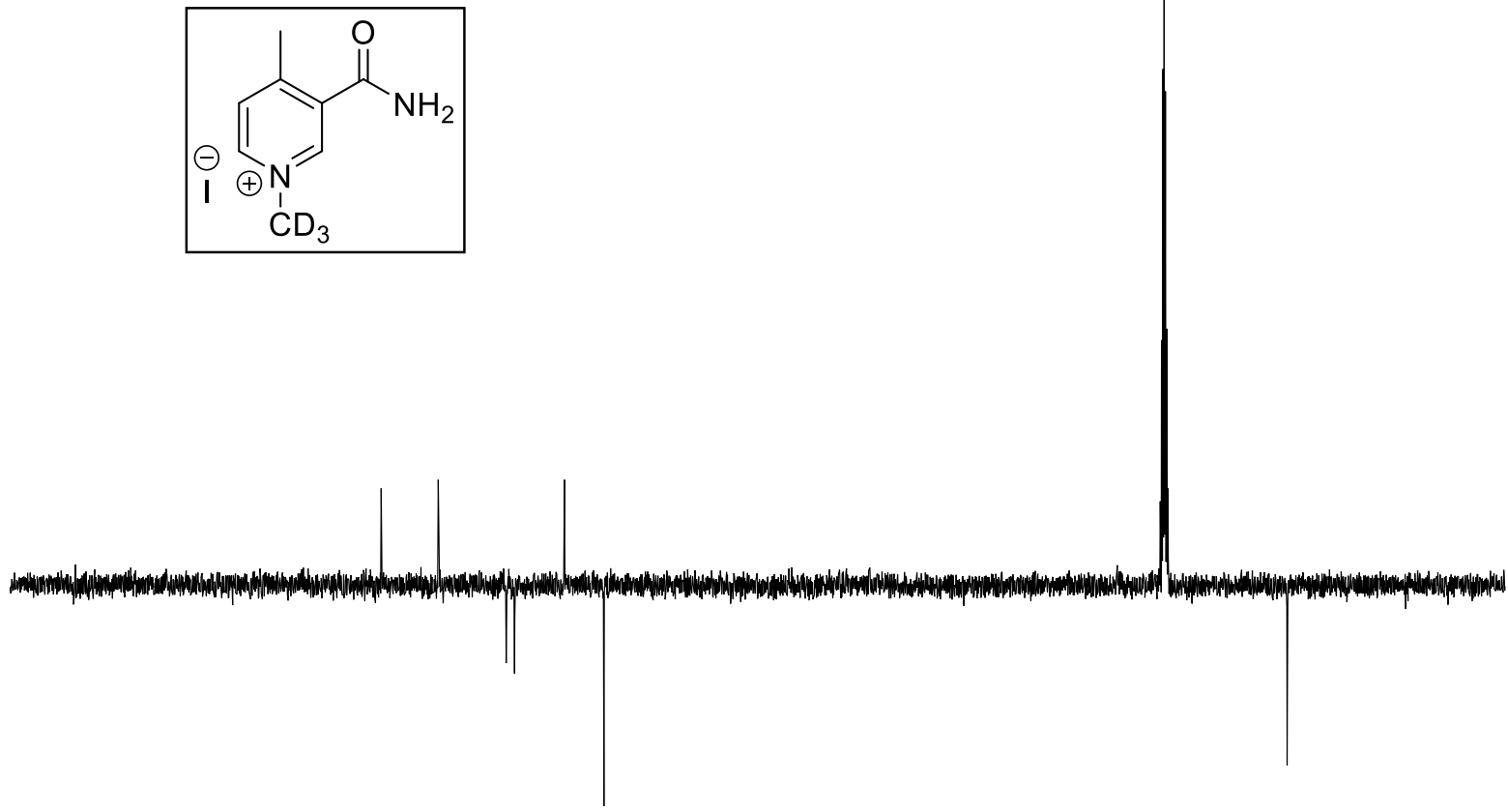

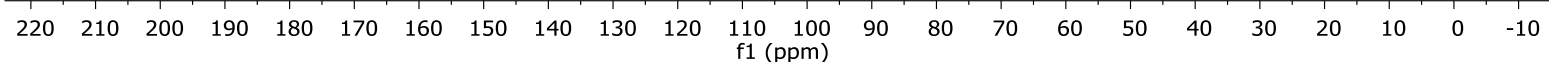


Compound 4b: ${ }^{1} \mathrm{H}$ NMR (400 MHz, $\left.\mathrm{D}_{2} \mathrm{O}\right)$
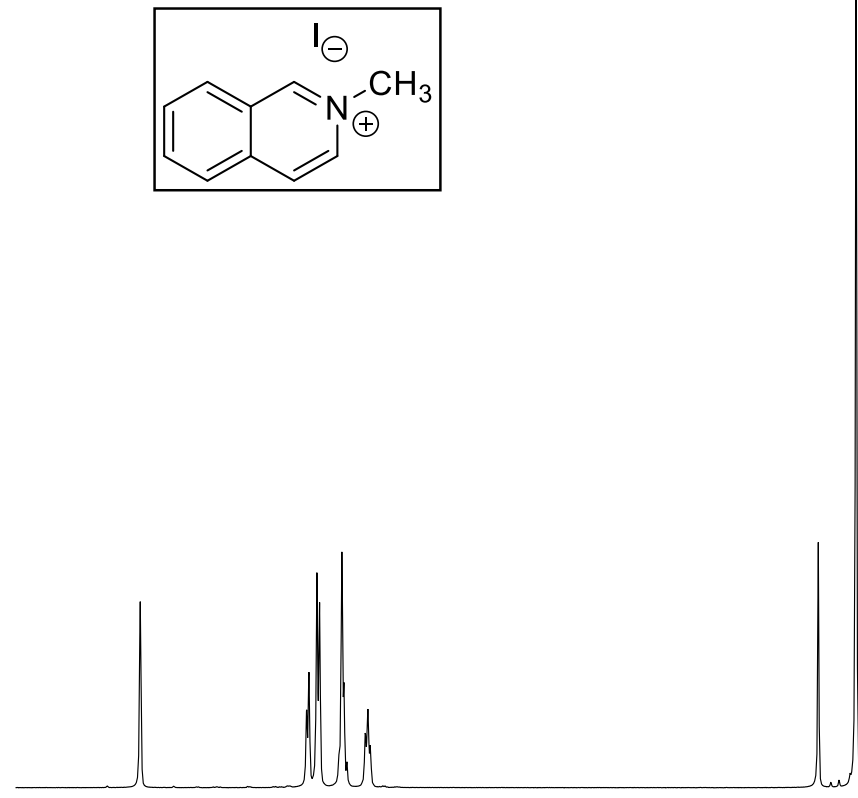

$\begin{array}{lllllllllllllllllllllll}10.5 & 10.0 & 9.5 & 9.0 & 8.5 & 8.0 & 7.5 & 7.0 & 6.5 & 6.0 & 5.5 & \begin{array}{c}5.0 \\ \text { f1 }\end{array} & 4.5 & 4.0 & 3.5 & 3.0 & 2.5 & 2.0 & 1.5 & 1.0 & 0.5 & 0.0 & -0 .\end{array}$

Compound 4b: ${ }^{13} \mathrm{C}$ NMR (101 MHz, $\left.\mathrm{D}_{2} \mathrm{O}\right)$

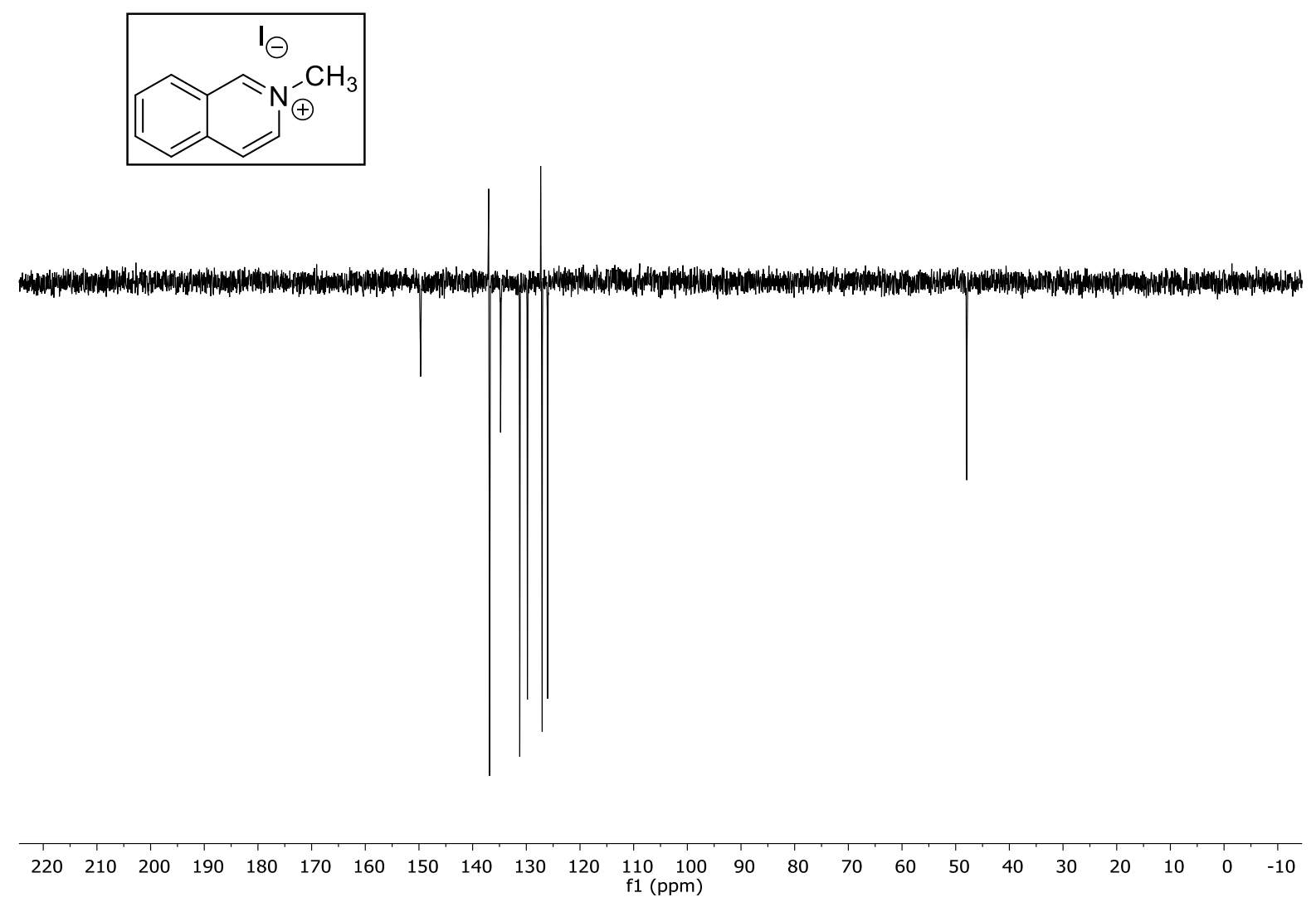


Compound 4c: ${ }^{1} \mathrm{H}$ NMR (400 MHz, $\left.\mathrm{D}_{2} \mathrm{O}\right)$
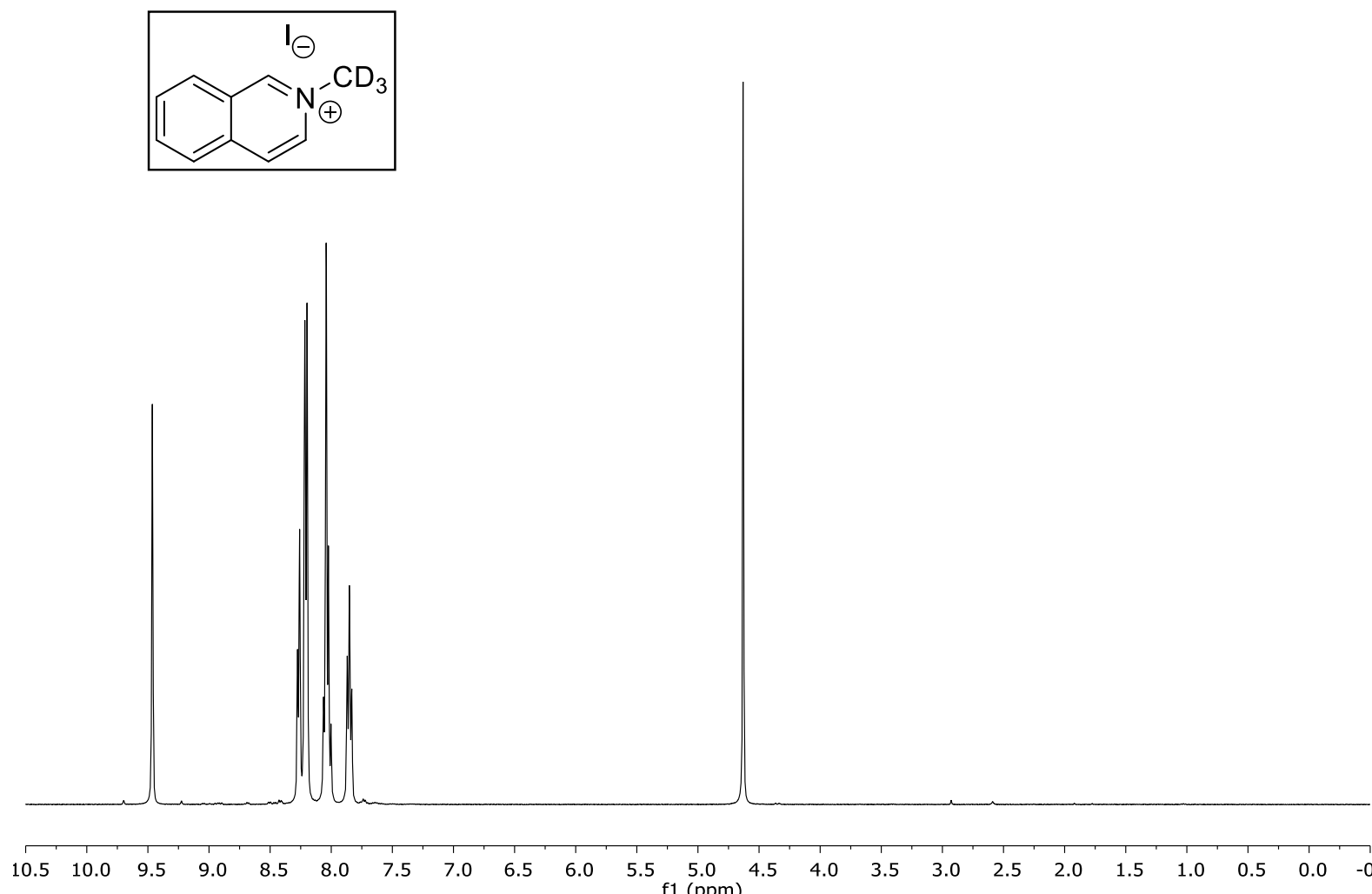

Compound 4c: ${ }^{13} \mathrm{C}$ NMR (101 MHz, $\left.\mathrm{D}_{2} \mathrm{O}\right)$
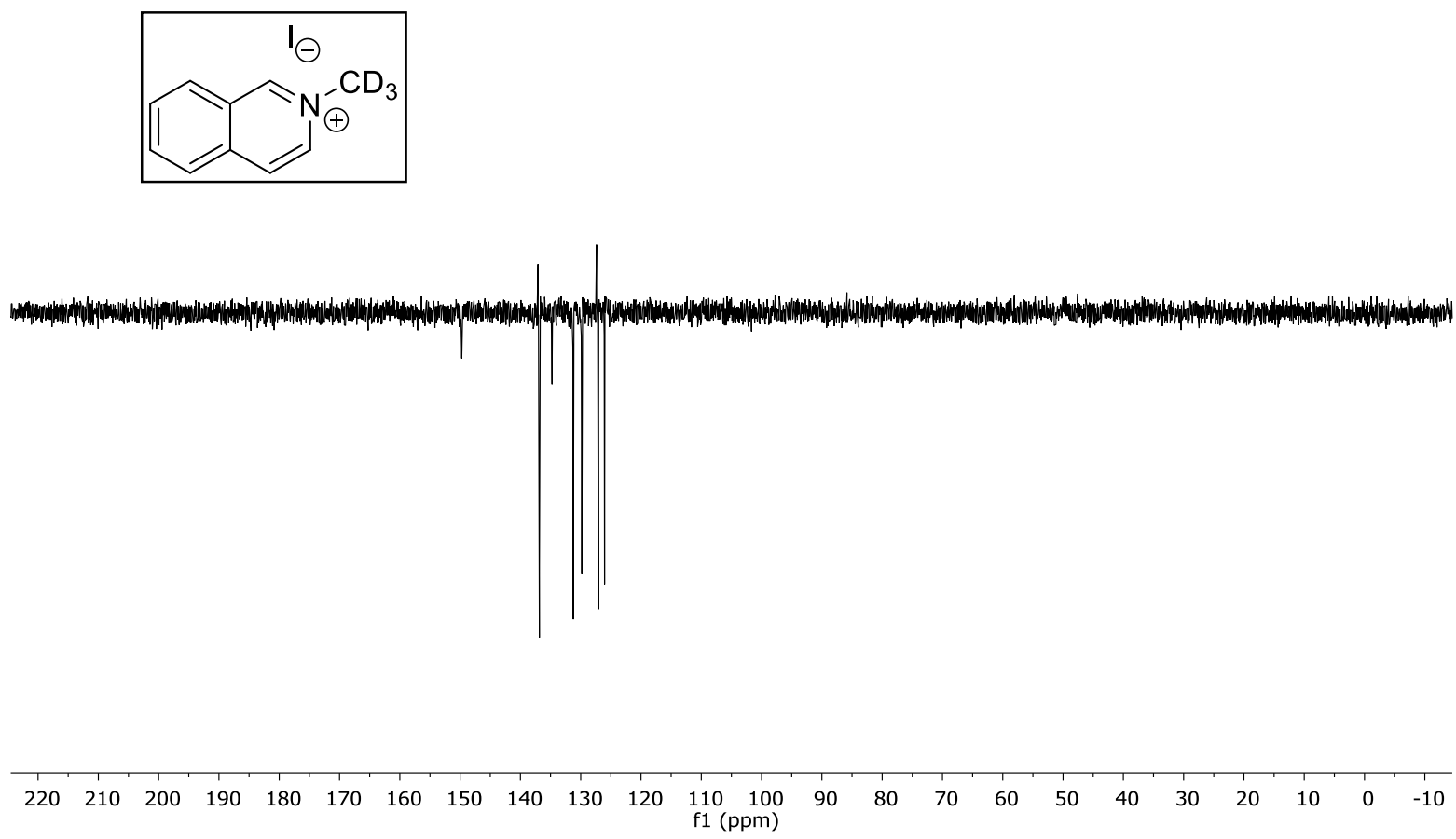
Compound 5b: ${ }^{1} \mathrm{H}$ NMR (400 MHz, DMSO-d 6$)$
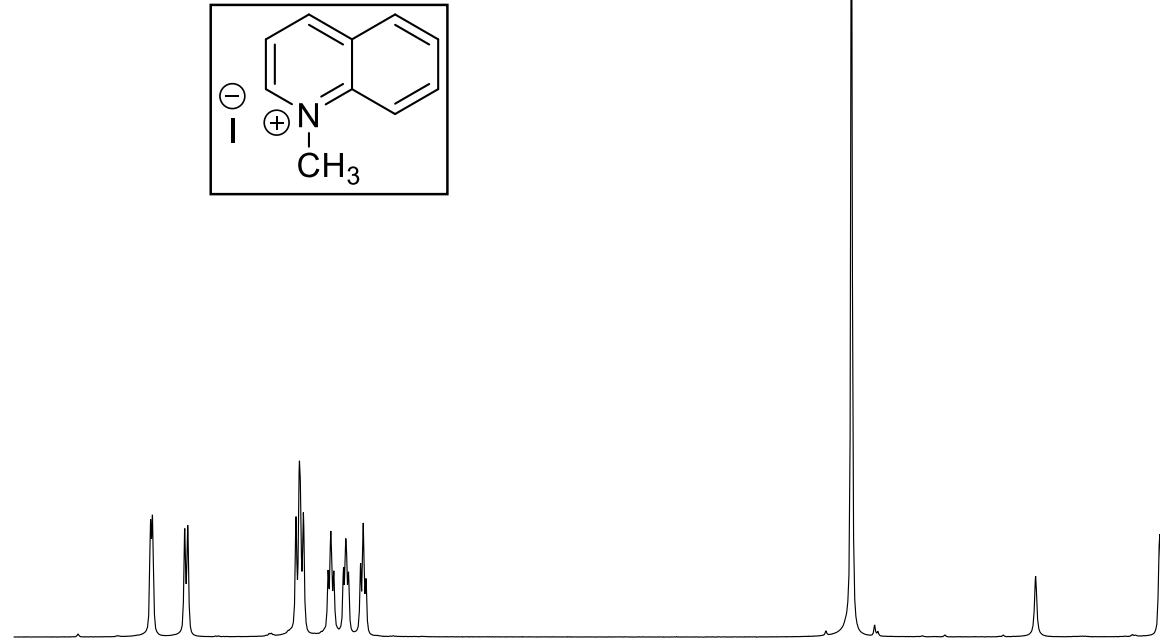

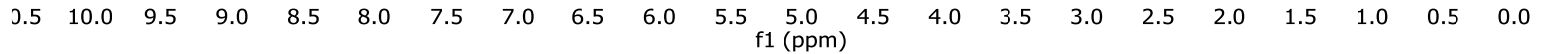

Compound 5b: ${ }^{13} \mathrm{C}$ NMR (101 MHz, DMSO-d 6 )
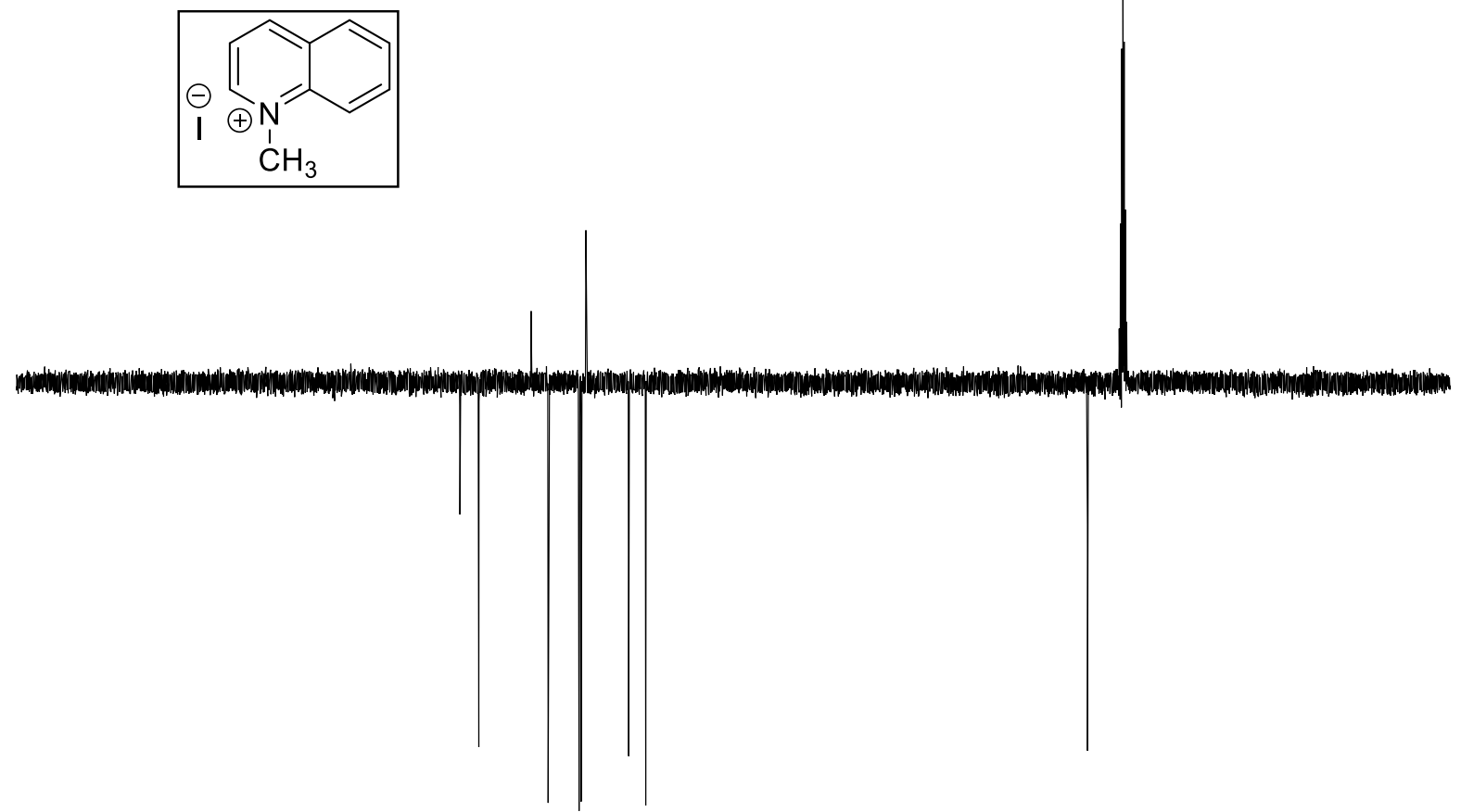

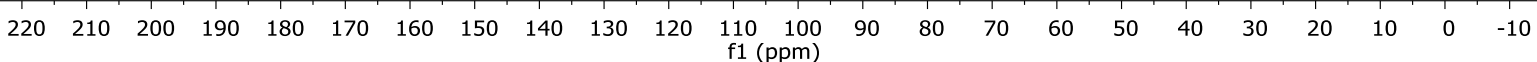


Compound 5c: ${ }^{1}$ H NMR (400 MHz, DMSO-d 6$)$
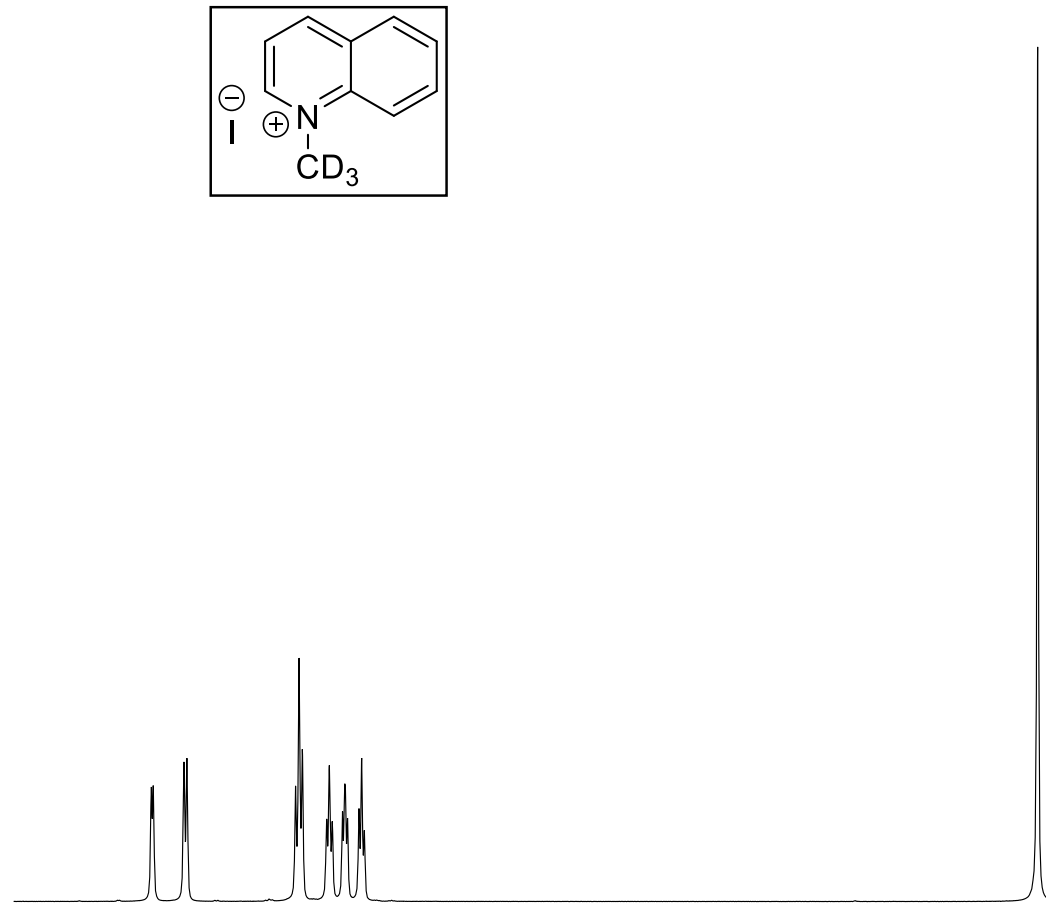

\begin{tabular}{llllllllllllllllllllllll}
\hline .5 & 10.0 & 9.5 & 9.0 & 8.5 & 8.0 & 7.5 & 7.0 & 6.5 & 6.0 & 5.5 & $\begin{array}{l}5.0 \\
\mathrm{f} 1(\mathrm{ppm})\end{array}$ & 4.5 & 4.0 & 3.5 & 3.0 & 2.5 & 2.0 & 1.5 & 1.0 & 0.5 & 0.0 & $-\mathrm{c}$
\end{tabular}

Compound 5c: ${ }^{13} \mathrm{C}$ NMR (101 MHz, DMSO-d 6$)$
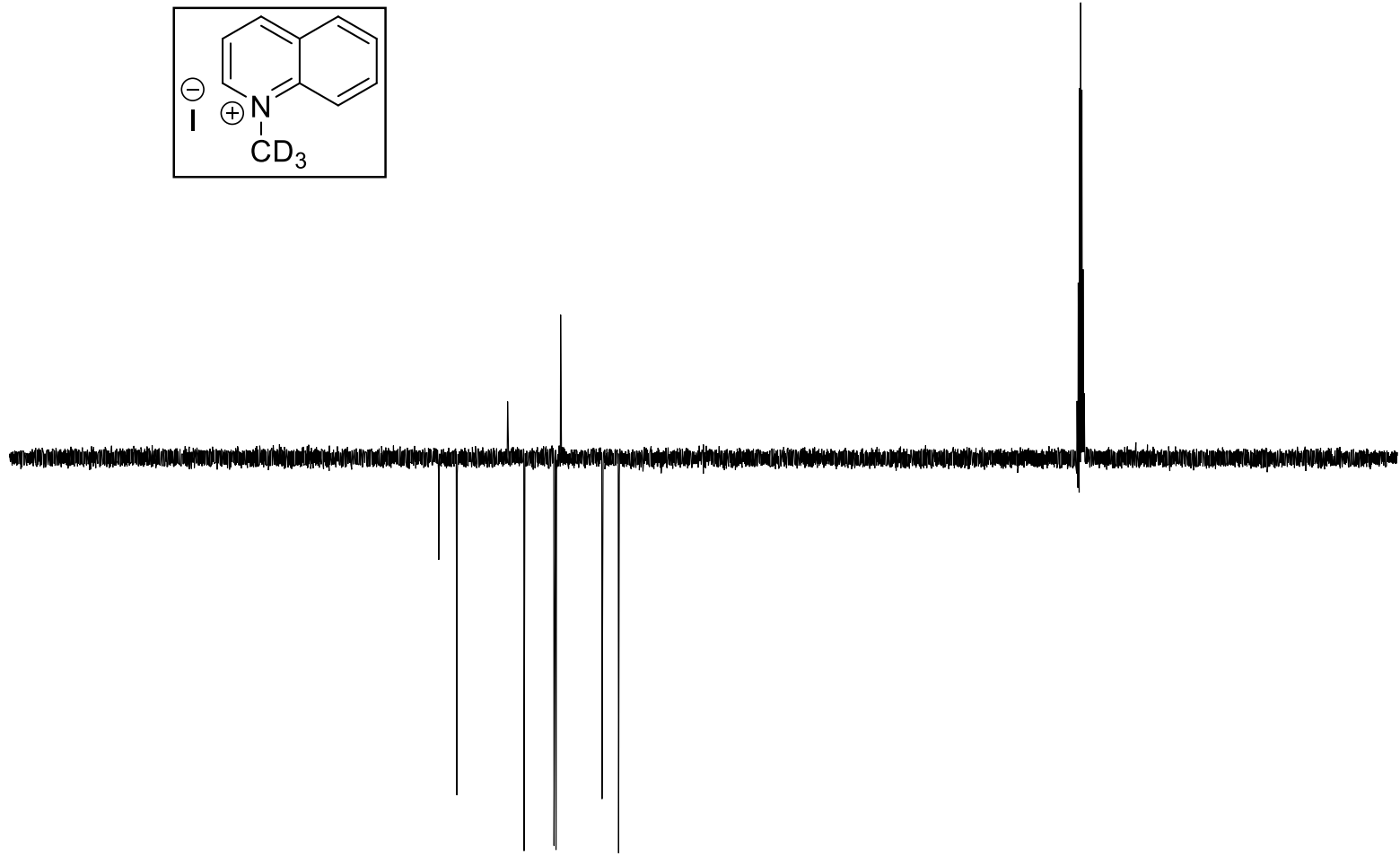

$\begin{array}{rllllllllllllllllllllll}220 & 210 & 200 & 190 & 180 & 170 & 160 & 150 & 140 & 130 & 120 & \underset{f 1}{110}(\mathrm{ppm}) & 90 & 80 & 70 & 60 & 50 & 40 & 30 & 20 & 10 & 0 & -10\end{array}$ 


\section{Compound $6 \mathrm{~b}^{1} \mathrm{H}$ NMR (400 $\mathrm{MHz}, \mathrm{CDCl}_{3}$ )}
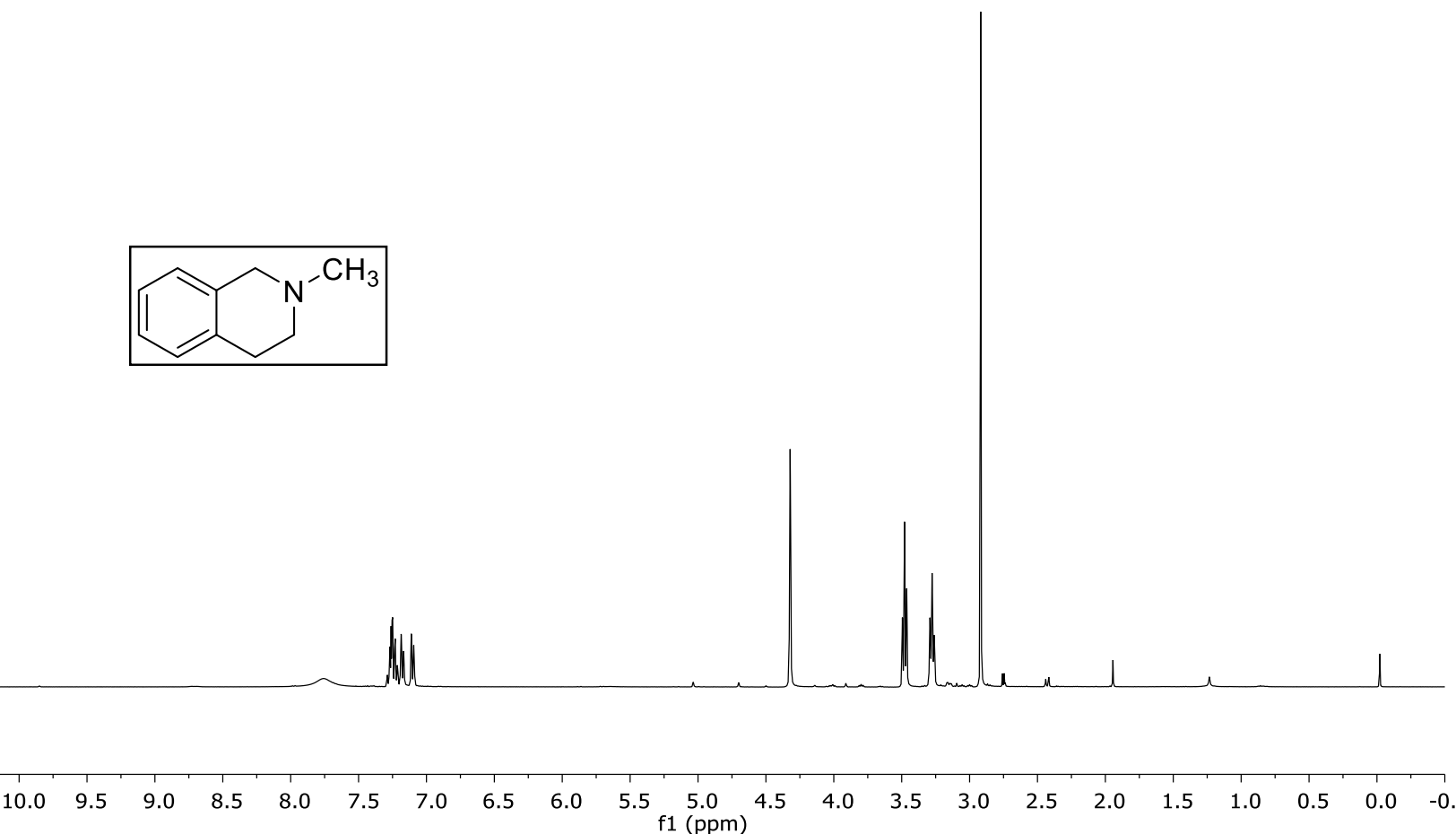

Compound 6b ${ }^{13} \mathrm{C}$ NMR (101 MHz, $\mathrm{CDCl}_{3}$ )

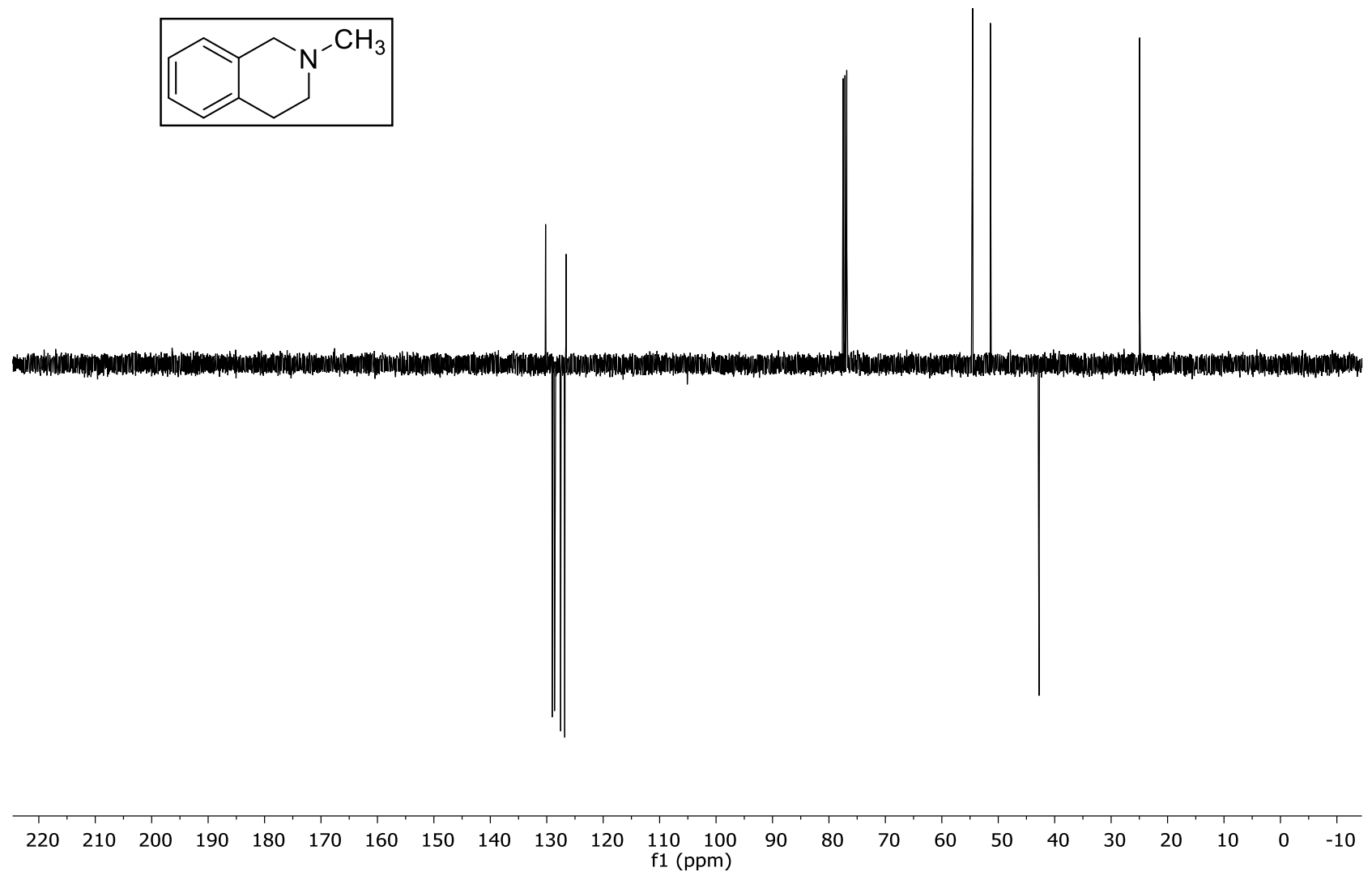


Compound $6 \mathrm{c}^{1} \mathrm{H}$ NMR (400 MHz, $\mathrm{CDCl}_{3}$ )

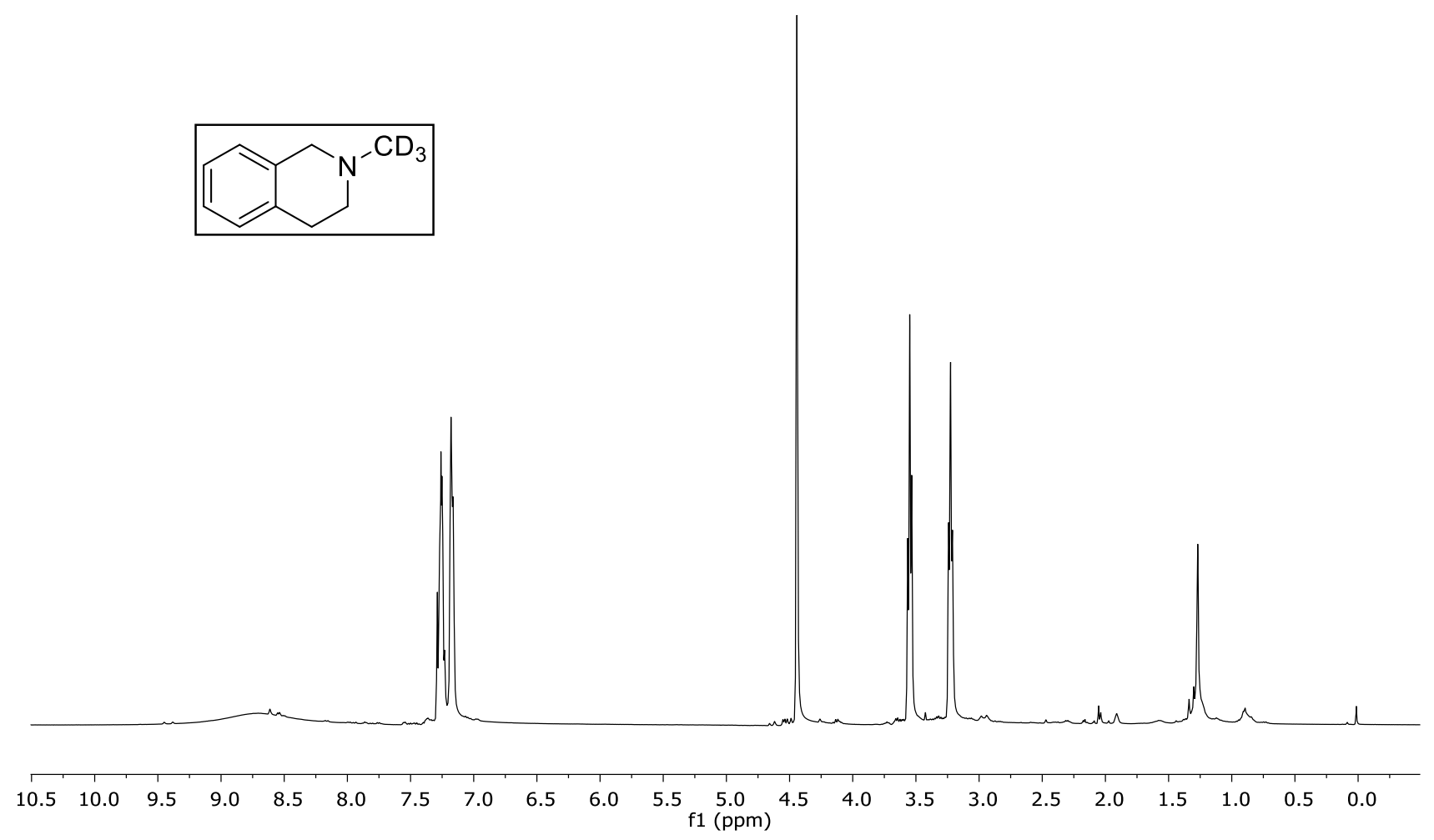

Compound $6 \mathrm{c}^{13} \mathrm{C}$ NMR (101 MHz, $\left.\mathrm{CDCl}_{3}\right)$

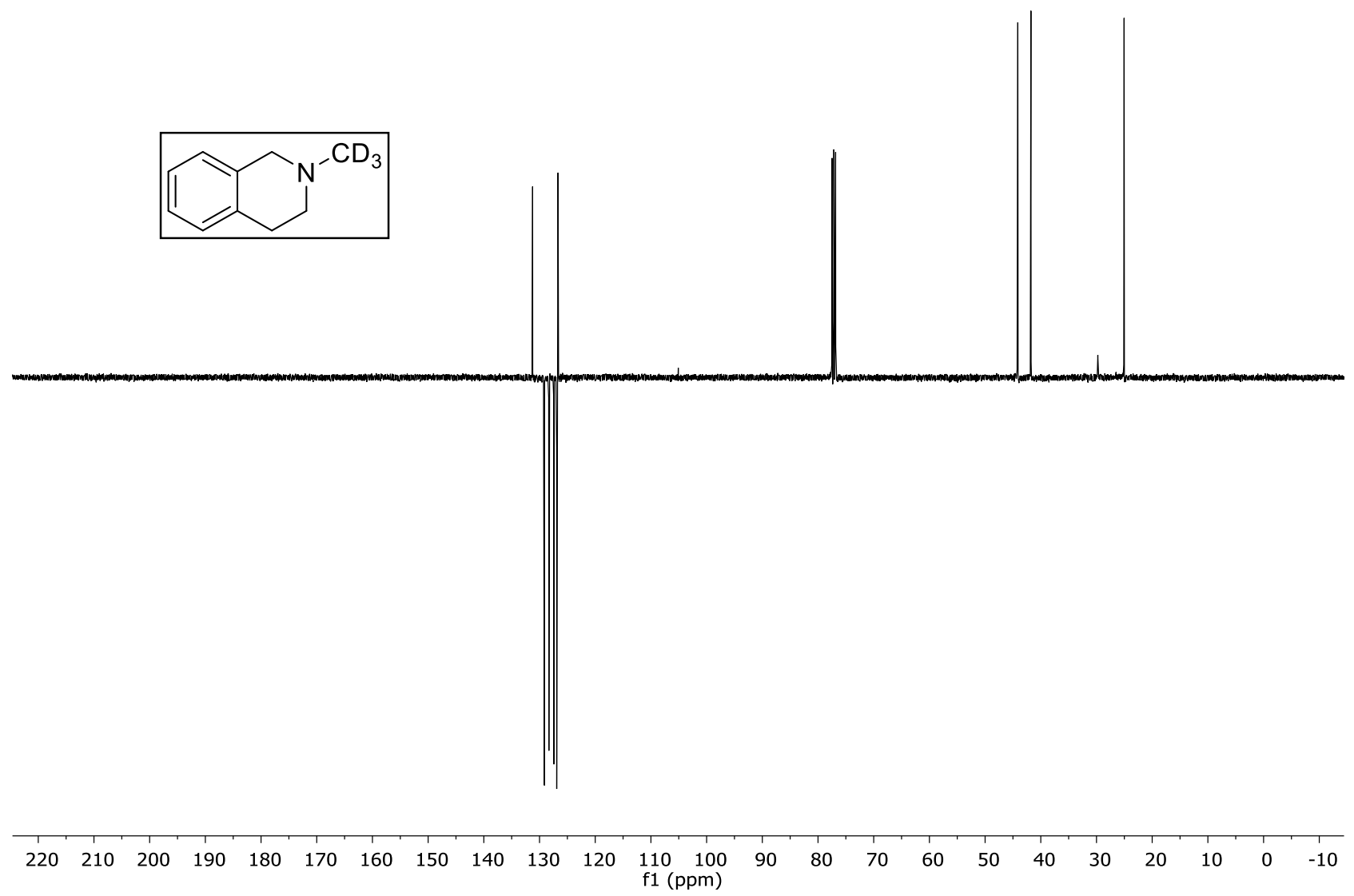


Compound 7b: ${ }^{1} \mathrm{H}$ NMR (400 MHz, DMSO-d 6$)$
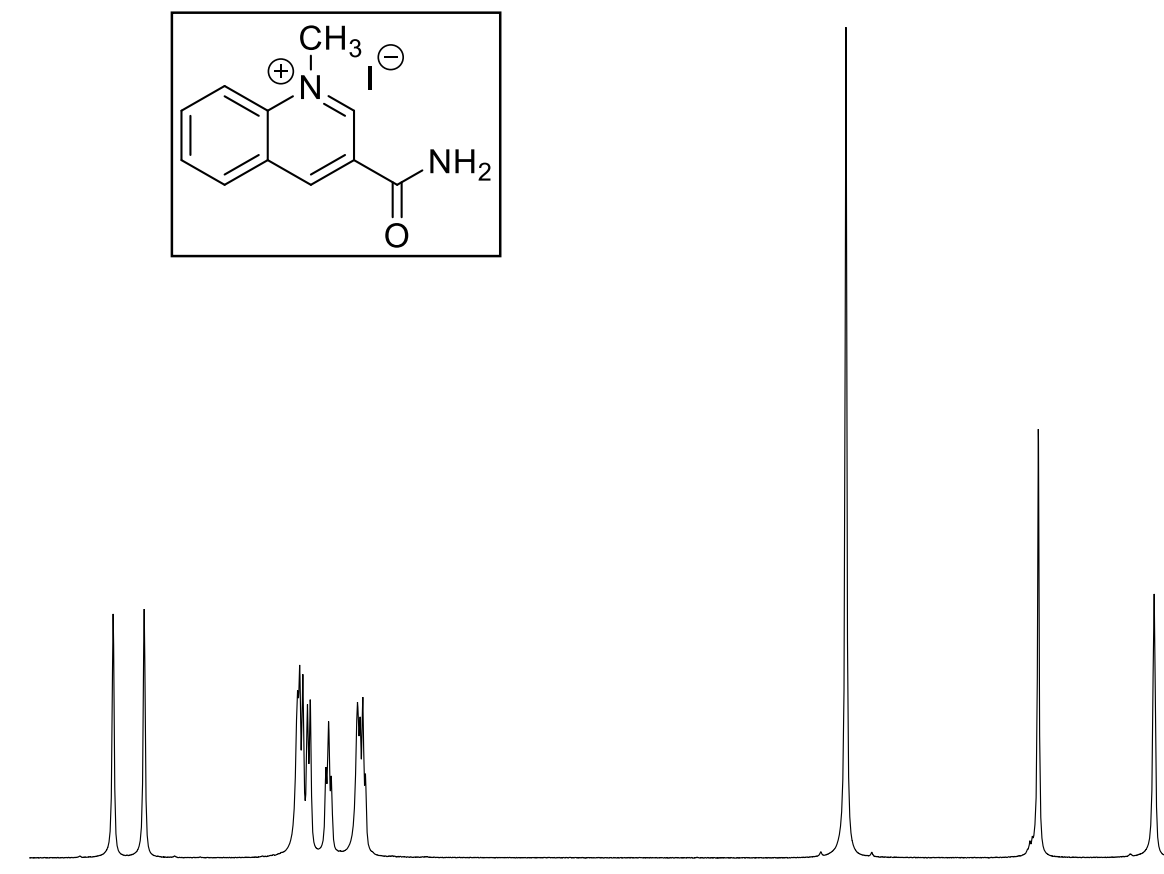

$\begin{array}{llllllllllllllllllllllllll}10.5 & 10.0 & 9.5 & 9.0 & 8.5 & 8.0 & 7.5 & 7.0 & 6.5 & 6.0 & 5.5 & 5.0 & 4.5 & 4.0 & 3.5 & 3.0 & 2.5 & 2.0 & 1.5 & 1.0 & 0.5 & 0.0 & -0 .\end{array}$

Compound 7b: ${ }^{13} \mathrm{C}$ NMR (101 MHz, DMSO-d $)$
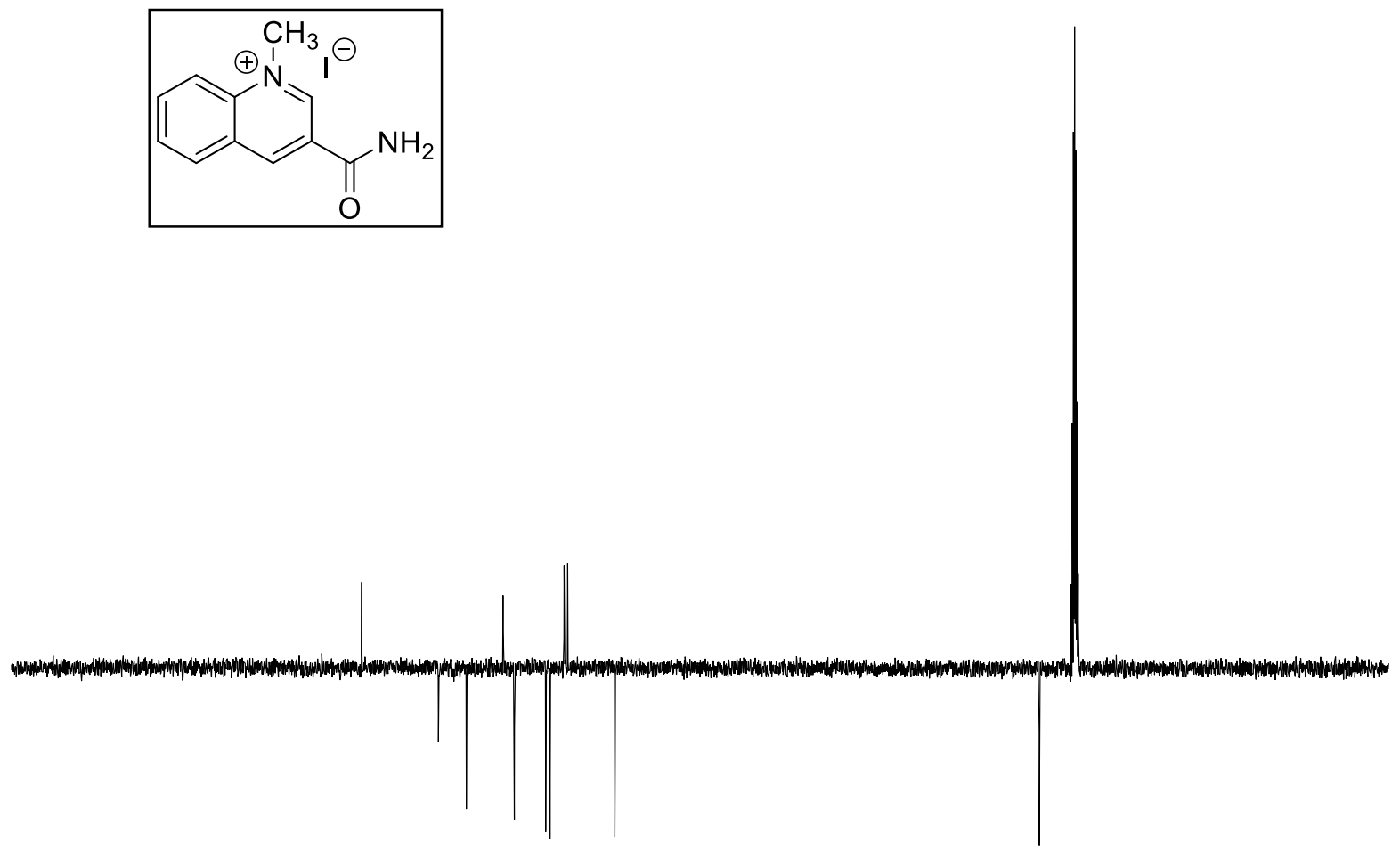

$\begin{array}{rllllllllllllllllllllll}220 & 210 & 200 & 190 & 180 & 170 & 160 & 150 & 140 & 130 & 120 & \begin{array}{l}110 \\ \mathrm{f} 1(\mathrm{ppm})\end{array} & 90 & 80 & 70 & 60 & 50 & 40 & 30 & 20 & 10 & 0 & -10\end{array}$ 
Compound 7c: ${ }^{1} \mathrm{H}$ NMR (400 MHz, DMSO-d 6$)$
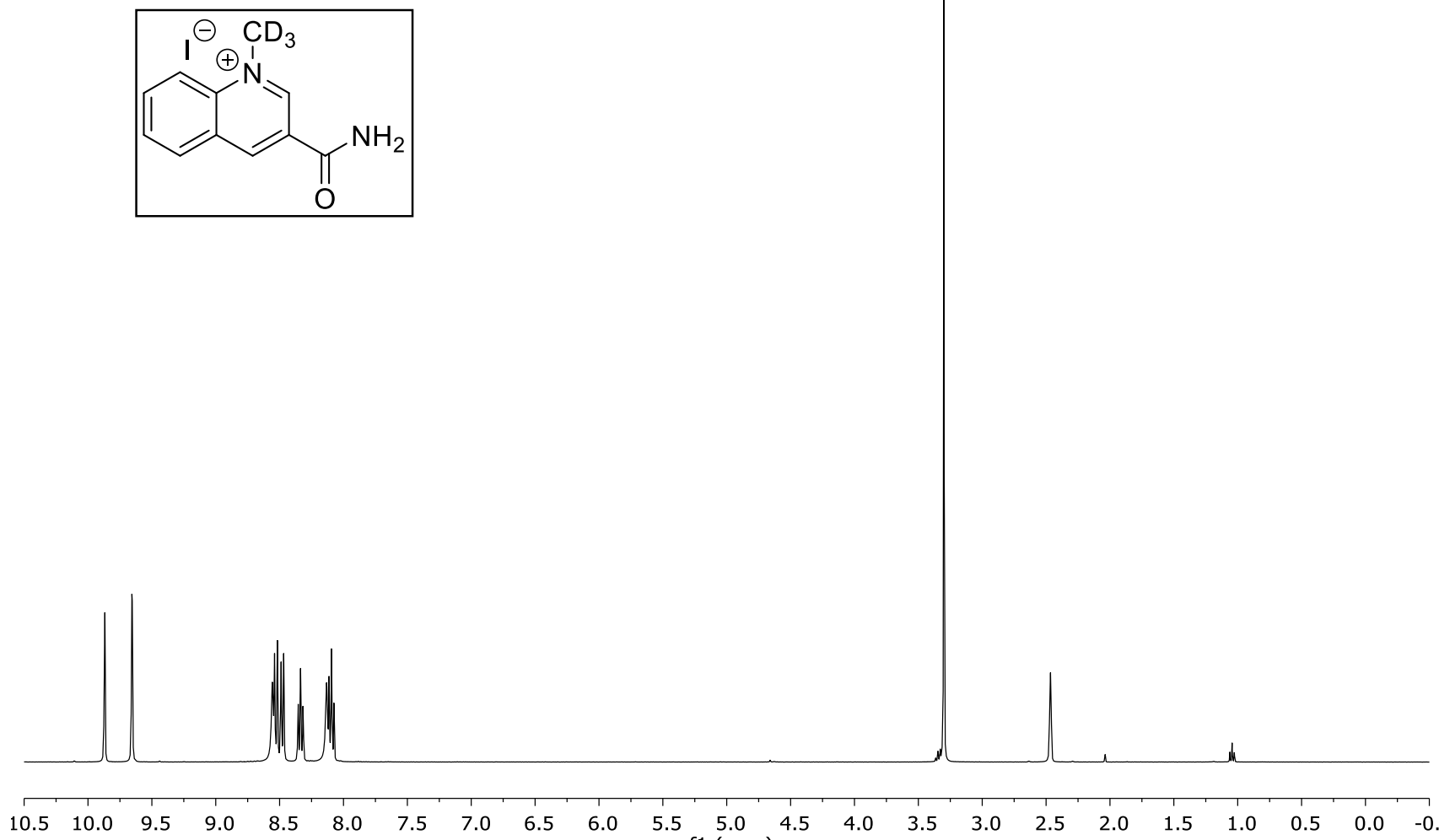

Compound 7c: ${ }^{13} \mathrm{C}$ NMR (101 MHz, DMSO-d 6$)$
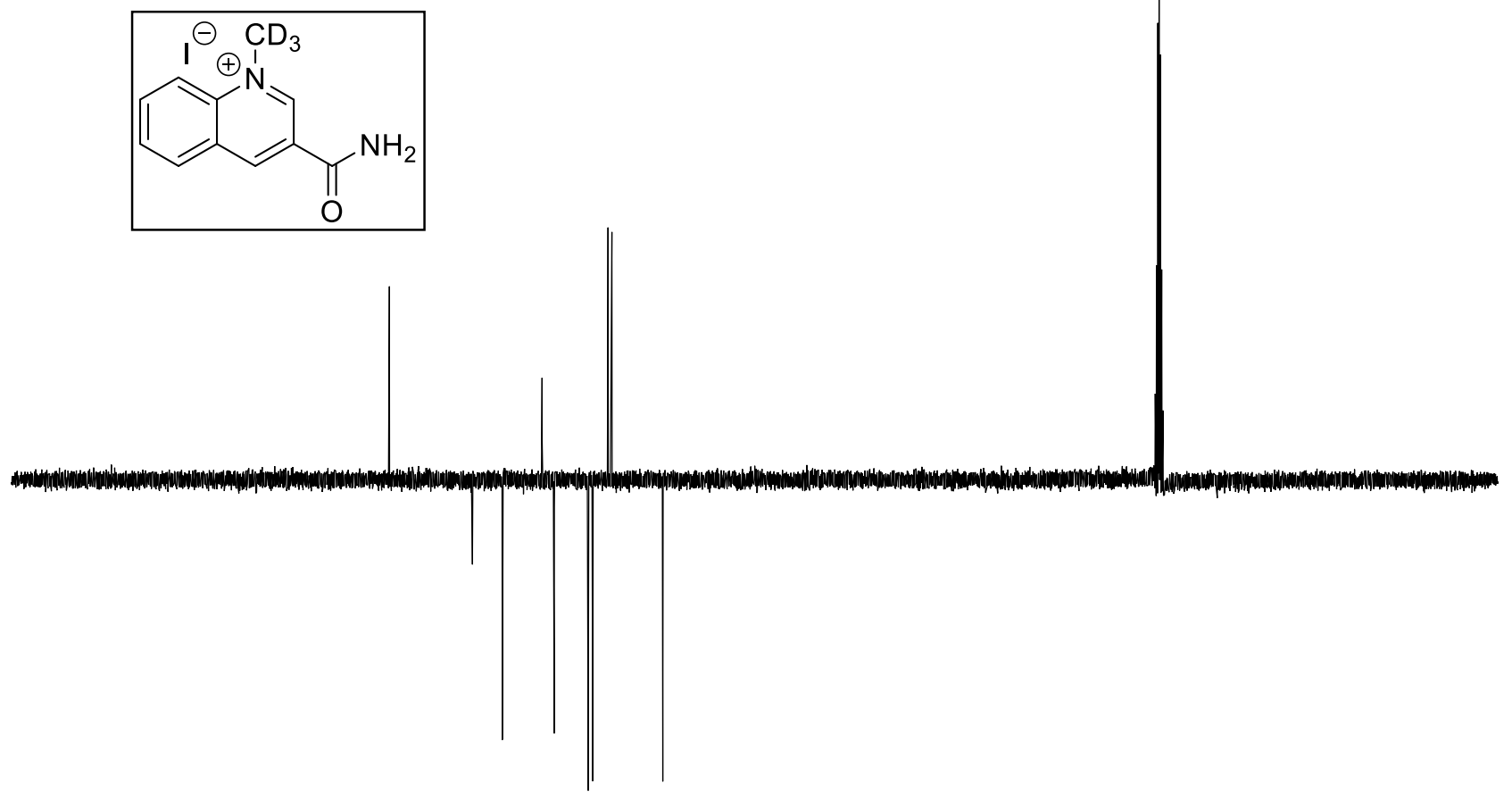

$\begin{array}{rllllllllllllllllllllll}220 & 210 & 200 & 190 & 180 & 170 & 160 & 150 & 140 & 130 & 120 & \begin{array}{c}110 \\ \mathrm{f} 1(\mathrm{ppm})\end{array} & 90 & 80 & 70 & 60 & 50 & 40 & 30 & 20 & 10 & 0 & -10\end{array}$ 
Compound 8b: ${ }^{1} \mathrm{H}$ NMR (400 MHz, $\left.\mathrm{D}_{2} \mathrm{O}\right)$
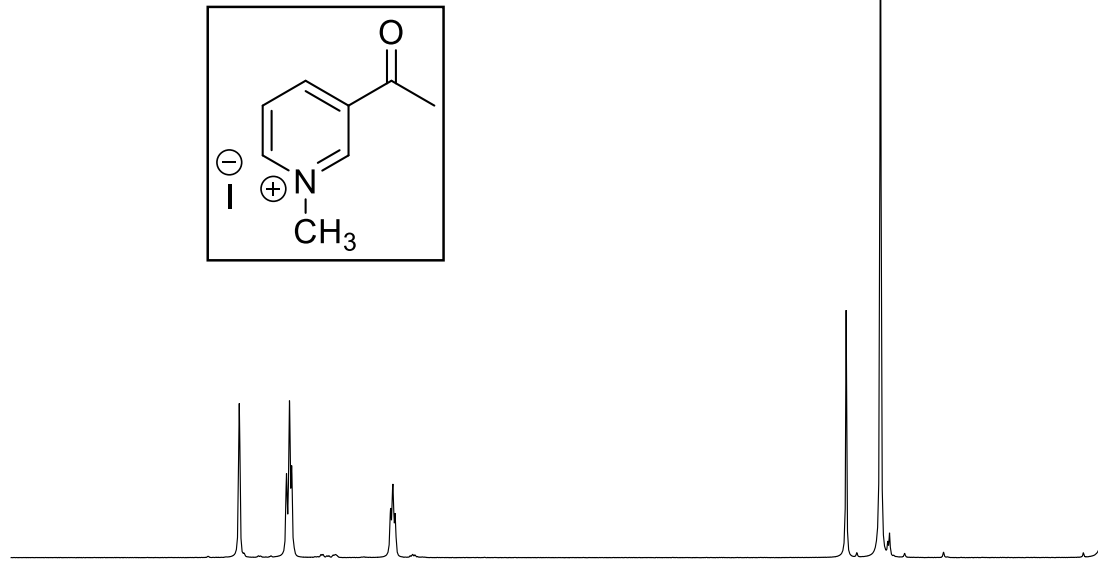

$\begin{array}{lllllllllllllllllllllll}11.0 & 10.5 & 10.0 & 9.5 & 9.0 & 8.5 & 8.0 & 7.5 & 7.0 & 6.5 & 6.0 & \underset{5}{5.5} \underset{(\mathrm{ppm})}{5.0} & 4.5 & 4.0 & 3.5 & 3.0 & 2.5 & 2.0 & 1.5 & 1.0 & 0.5 & 0.0 & -0.5\end{array}$

Compound 8b: ${ }^{13} \mathrm{C}$ NMR (101 MHz, $\left.\mathrm{D}_{2} \mathrm{O}\right)$
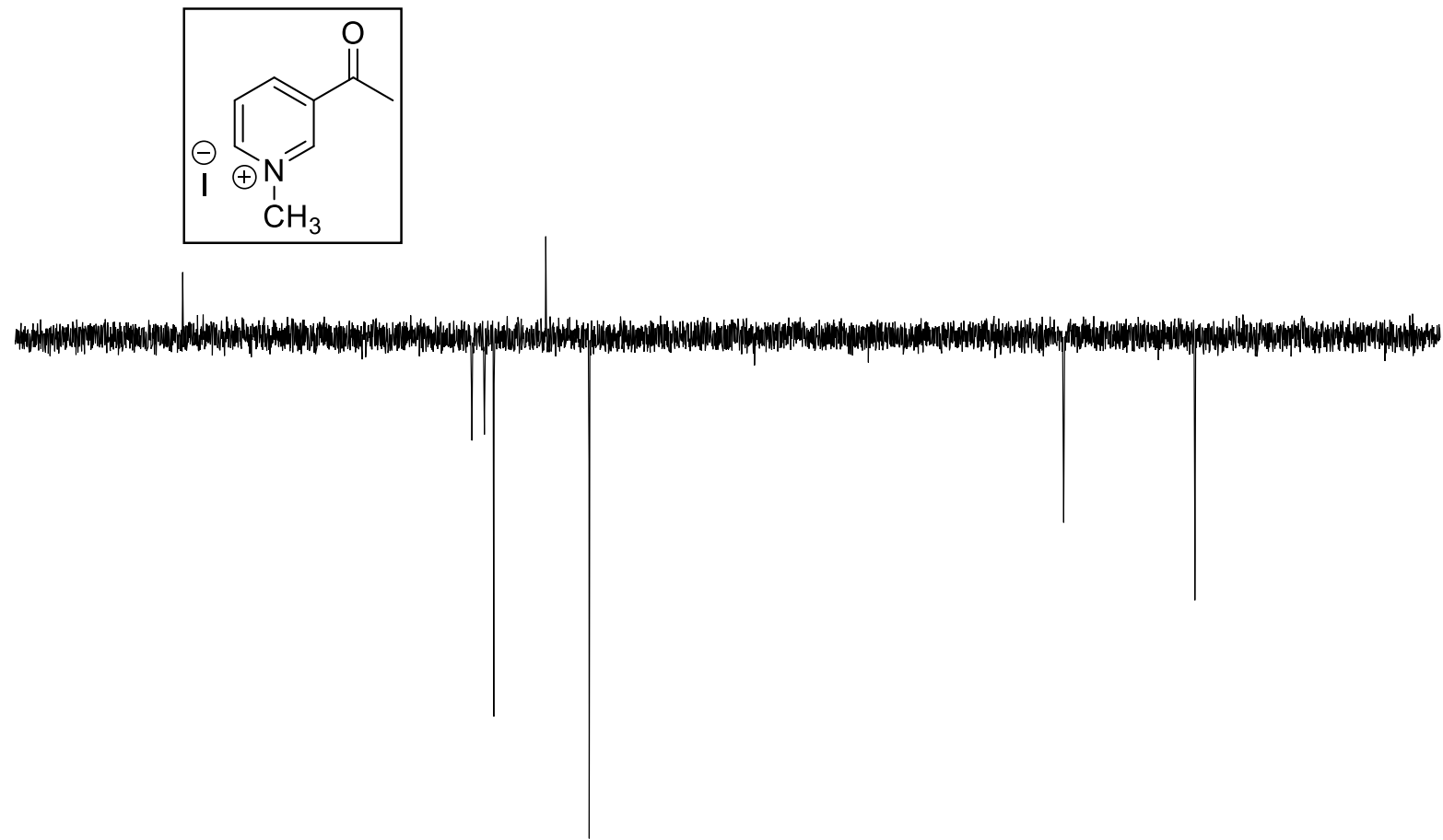

$\begin{array}{lllllllllllllllllllllll}220 & 210 & 200 & 190 & 180 & 170 & 160 & 150 & 140 & 130 & 120 & \begin{array}{c}110 \\ \mathrm{f} 1(\mathrm{ppm})\end{array} & 100 & 80 & 70 & 60 & 50 & 40 & 30 & 20 & 10 & 0 & -10\end{array}$ 
Compound 8c: ${ }^{1} \mathrm{H}$ NMR (400 MHz, $\left.\mathrm{D}_{2} \mathrm{O}\right)$
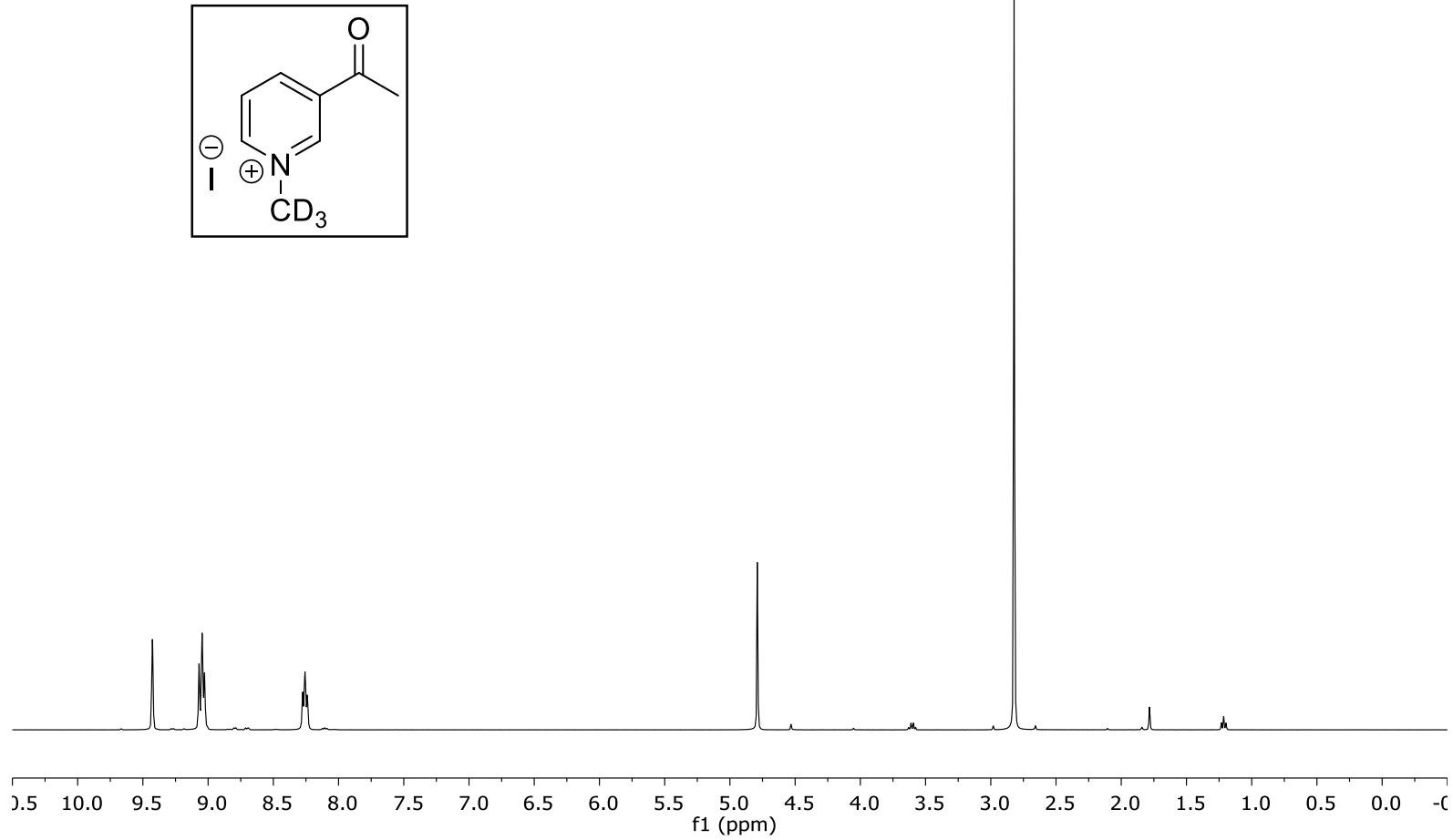

Compound 8c: ${ }^{13} \mathrm{C}$ NMR (101 MHz, $\left.\mathrm{D}_{2} \mathrm{O}\right)$

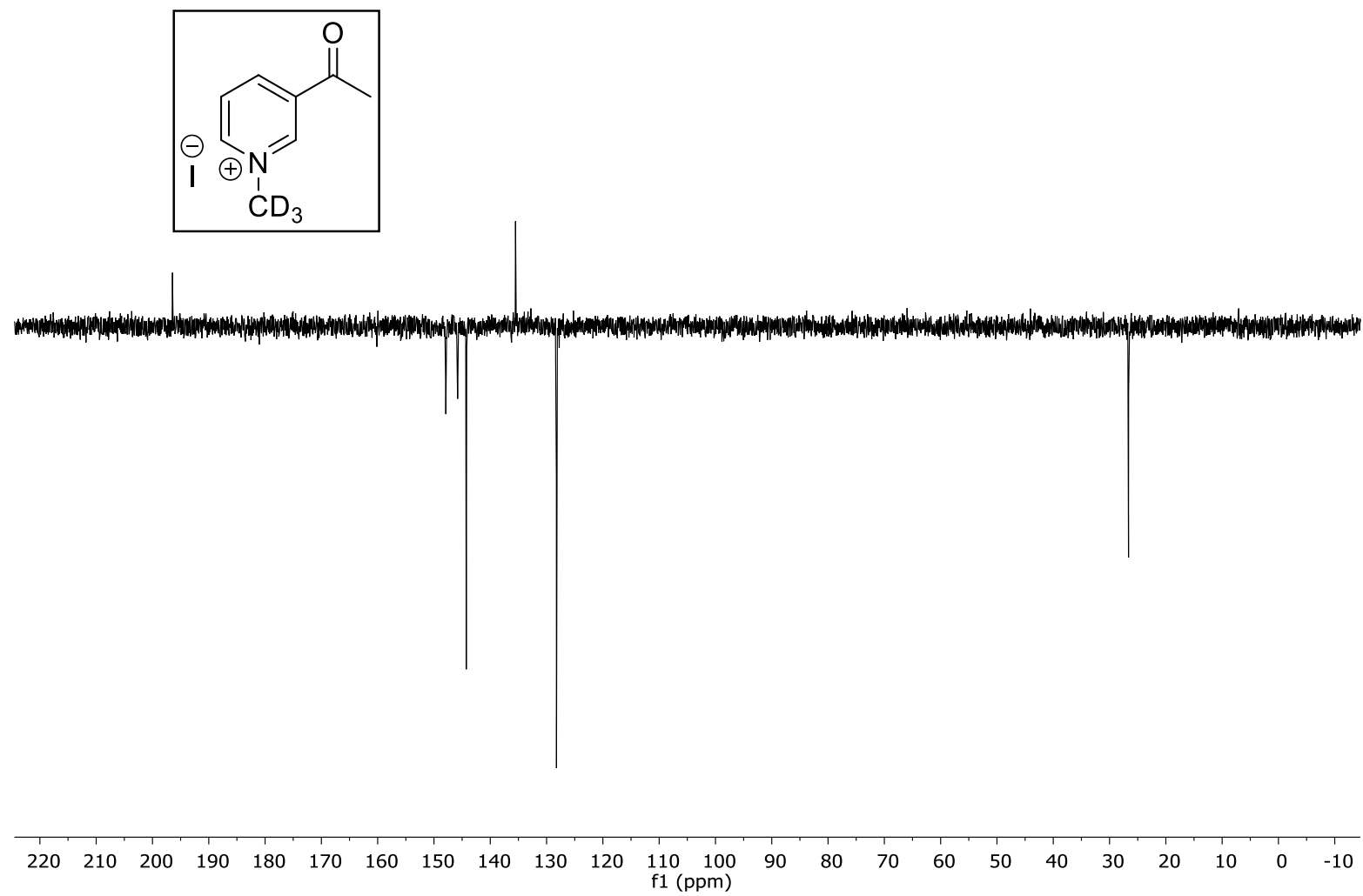


Compound 9b: ${ }^{1} \mathrm{H}$ NMR (400 MHz, DMSO- $\left.d_{6}\right)$
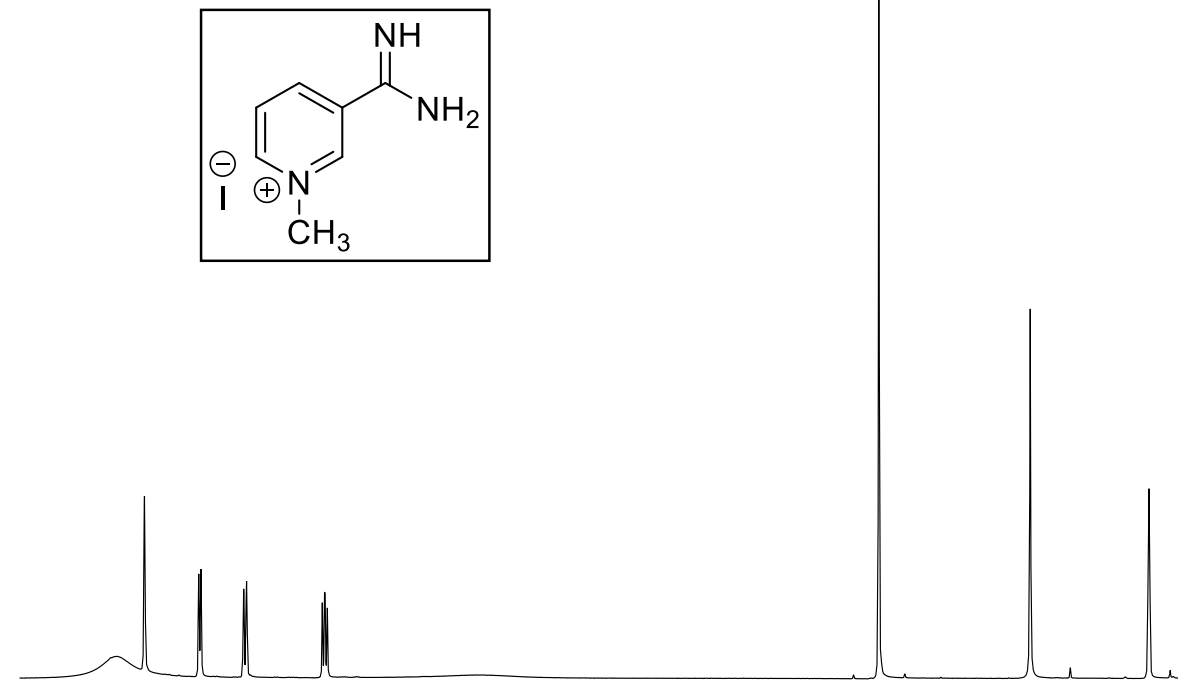

$\begin{array}{lllllllllllllllllllllll}10.5 & 10.0 & 9.5 & 9.0 & 8.5 & 8.0 & 7.5 & 7.0 & 6.5 & 6.0 & 5.5 \underset{\mathrm{f} 1}{5.0}(\mathrm{ppm}) & 4.5 & 4.0 & 3.5 & 3.0 & 2.5 & 2.0 & 1.5 & 1.0 & 0.5 & 0.0 & -0 .\end{array}$

Compound 9b: ${ }^{13} \mathrm{C}$ NMR (101 MHz, DMSO- $\left.d_{6}\right)$<smiles></smiles>

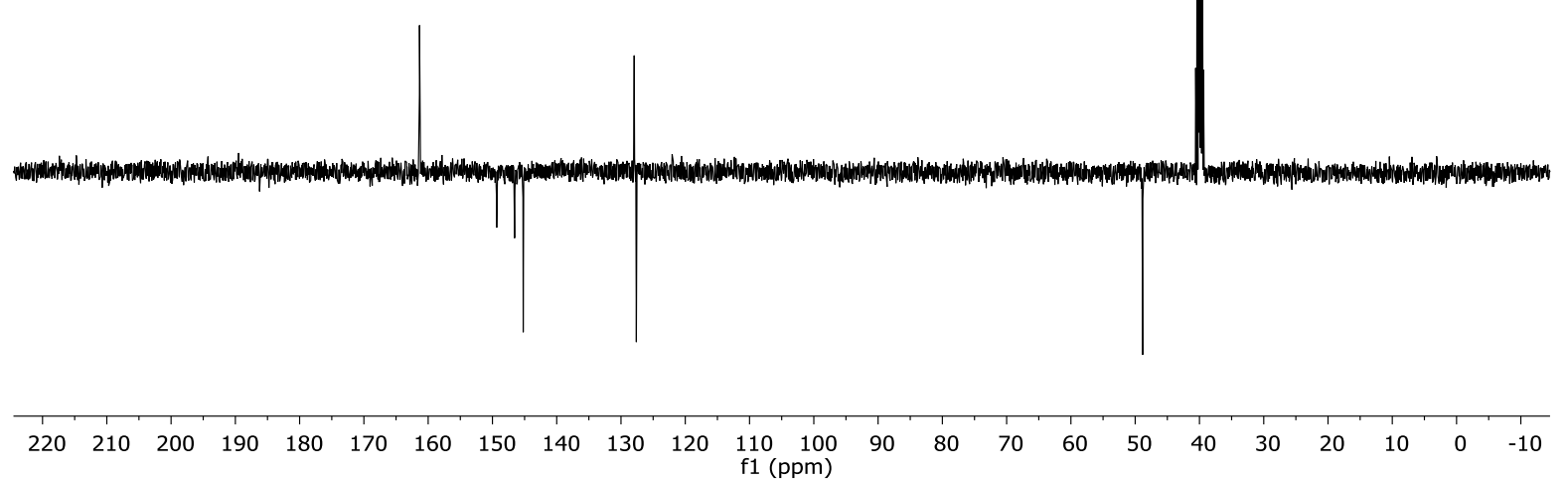


Compound 9c: ${ }^{1} \mathrm{H}$ NMR (400 MHz, DMSO- $\left.d_{6}\right)$
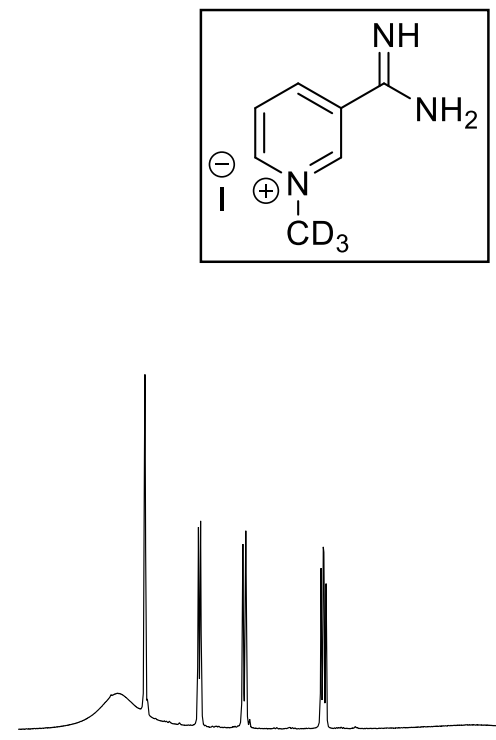

$\begin{array}{lllllllllllllllllllllll}10.5 & 10.0 & 9.5 & 9.0 & 8.5 & 8.0 & 7.5 & 7.0 & 6.5 & 6.0 & 5.5 & \begin{array}{c}5.0 \\ \mathrm{f} 1(\mathrm{ppm})\end{array} & 4.5 & 4.0 & 3.5 & 3.0 & 2.5 & 2.0 & 1.5 & 1.0 & 0.5 & 0.0 & -0 .\end{array}$

Compound 9c: ${ }^{13} \mathrm{C}$ NMR (101 MHz, DMSO-d $)$
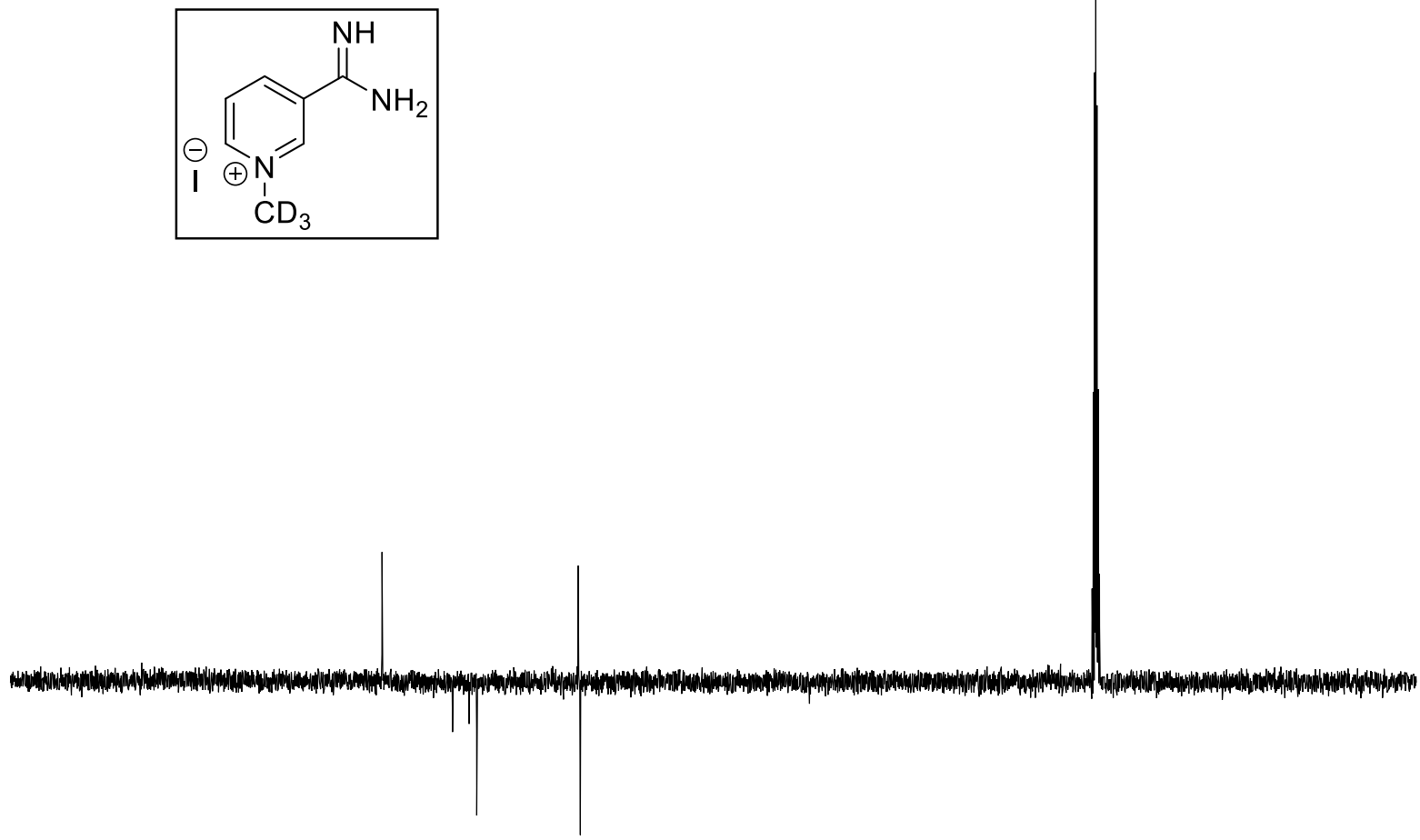

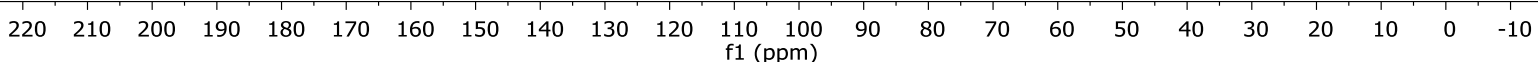


Compound 10b: ${ }^{1} \mathrm{H}$ NMR (400 MHz, $\left.\mathrm{D}_{2} \mathrm{O}\right)$
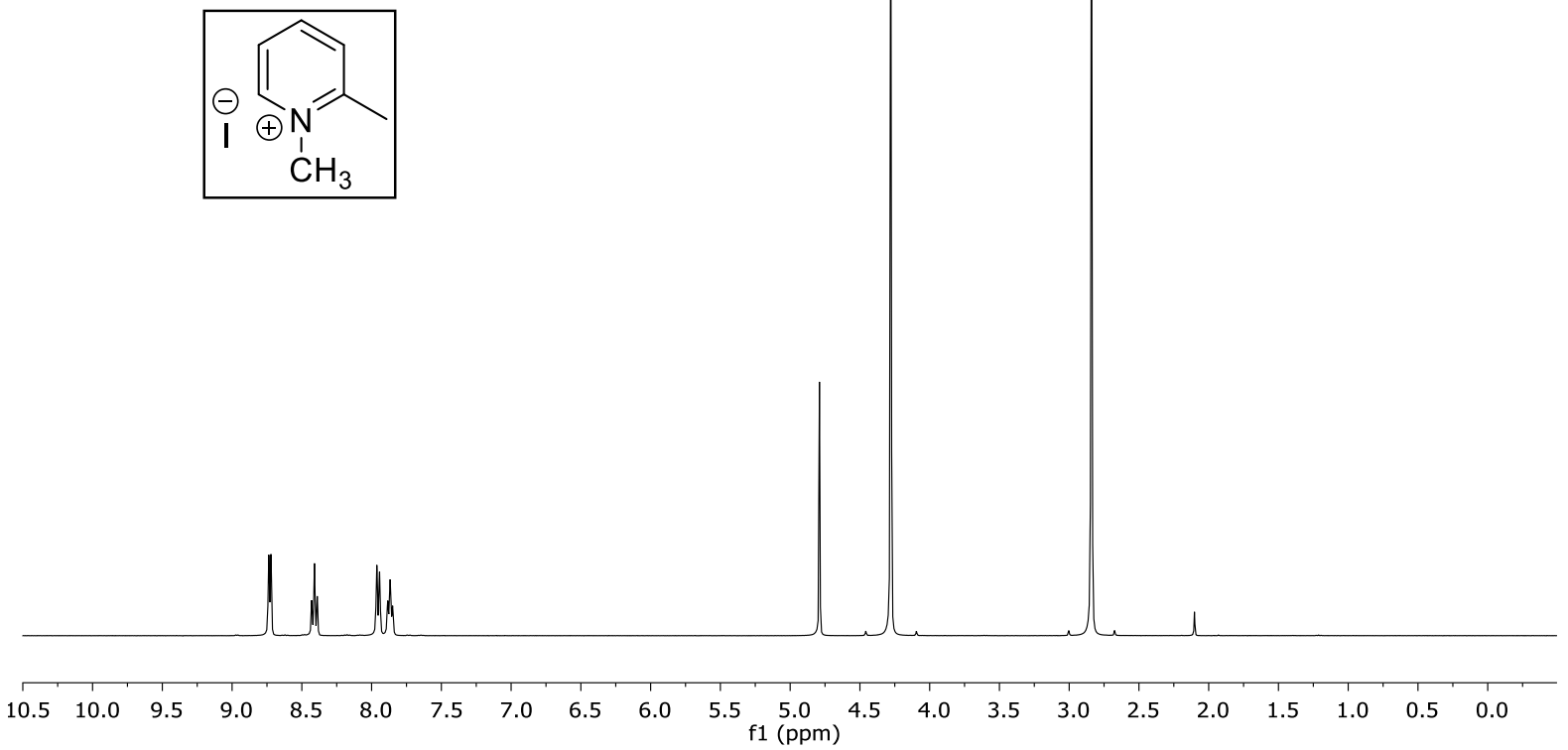

Compound 10b: ${ }^{13} \mathrm{C}$ NMR (101 MHz, $\left.\mathrm{D}_{2} \mathrm{O}\right)$
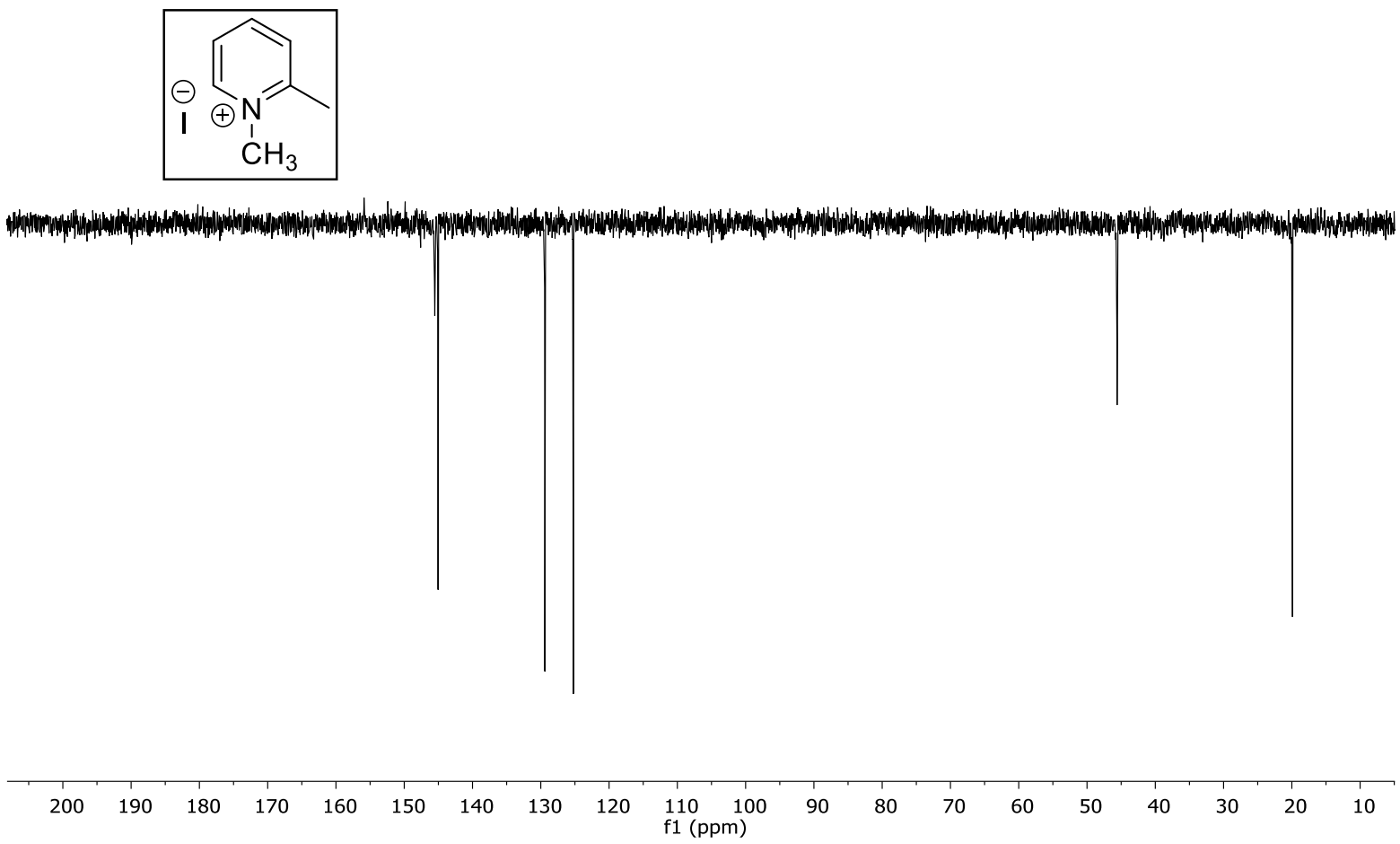

S33 
Compound 10c: ${ }^{1} \mathrm{H}$ NMR (400 MHz, $\left.\mathrm{D}_{2} \mathrm{O}\right)$
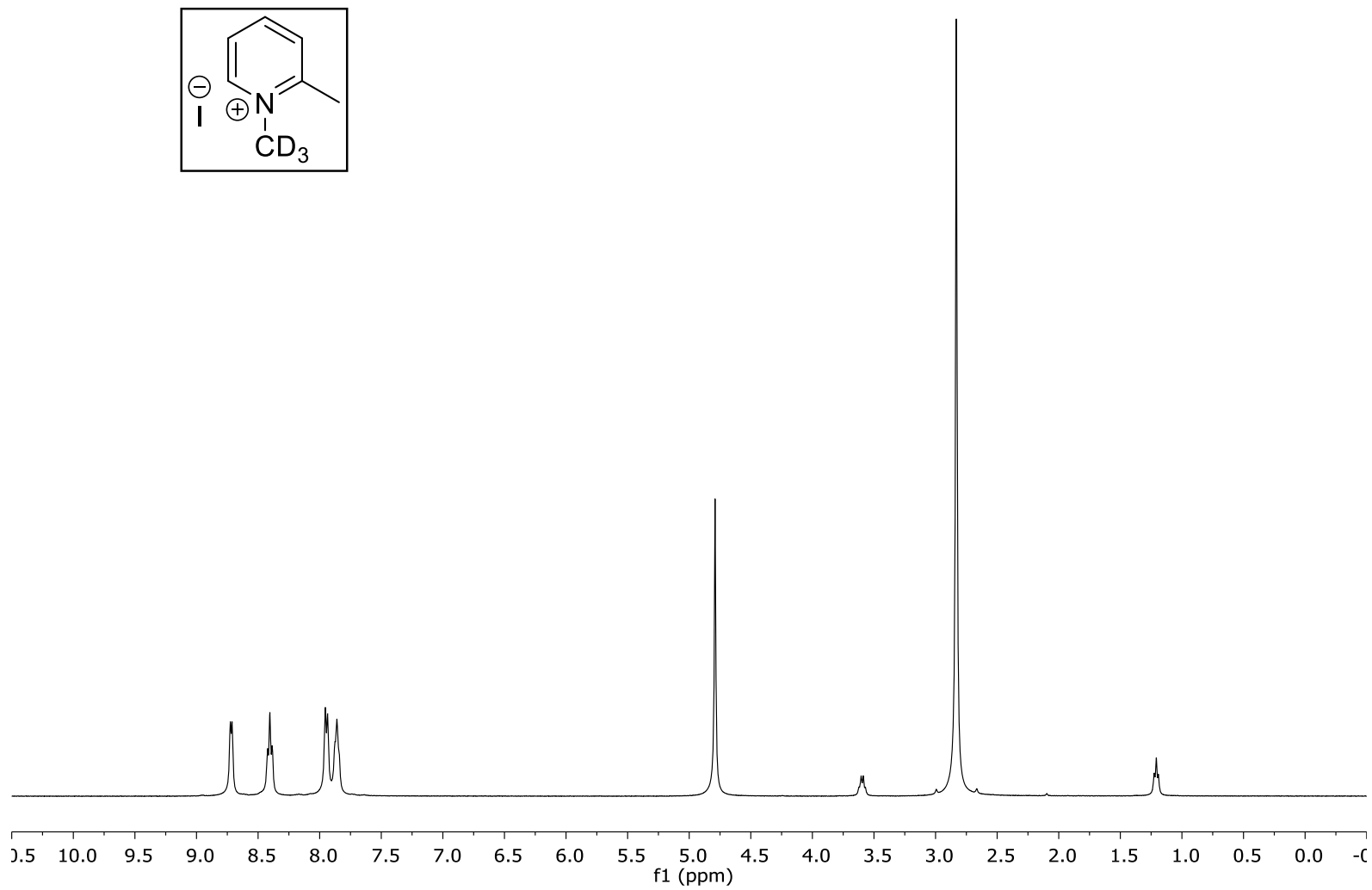

Compound 10c: ${ }^{13} \mathrm{C}$ NMR (101 MHz, $\left.\mathrm{D}_{2} \mathrm{O}\right)$
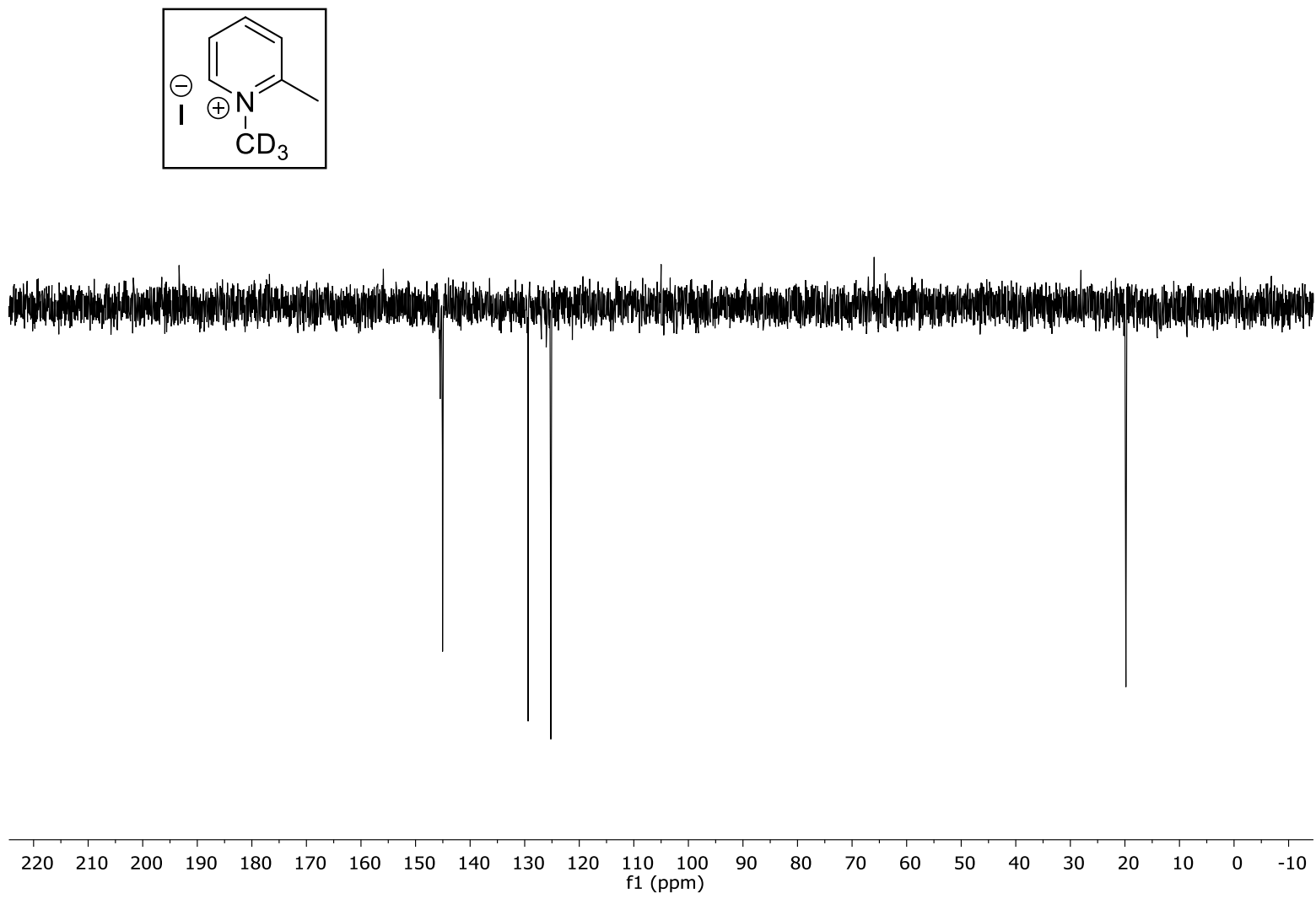
Compound 11b: ${ }^{1} \mathrm{H}$ NMR (400 MHz, $\left.\mathrm{D}_{2} \mathrm{O}\right)$

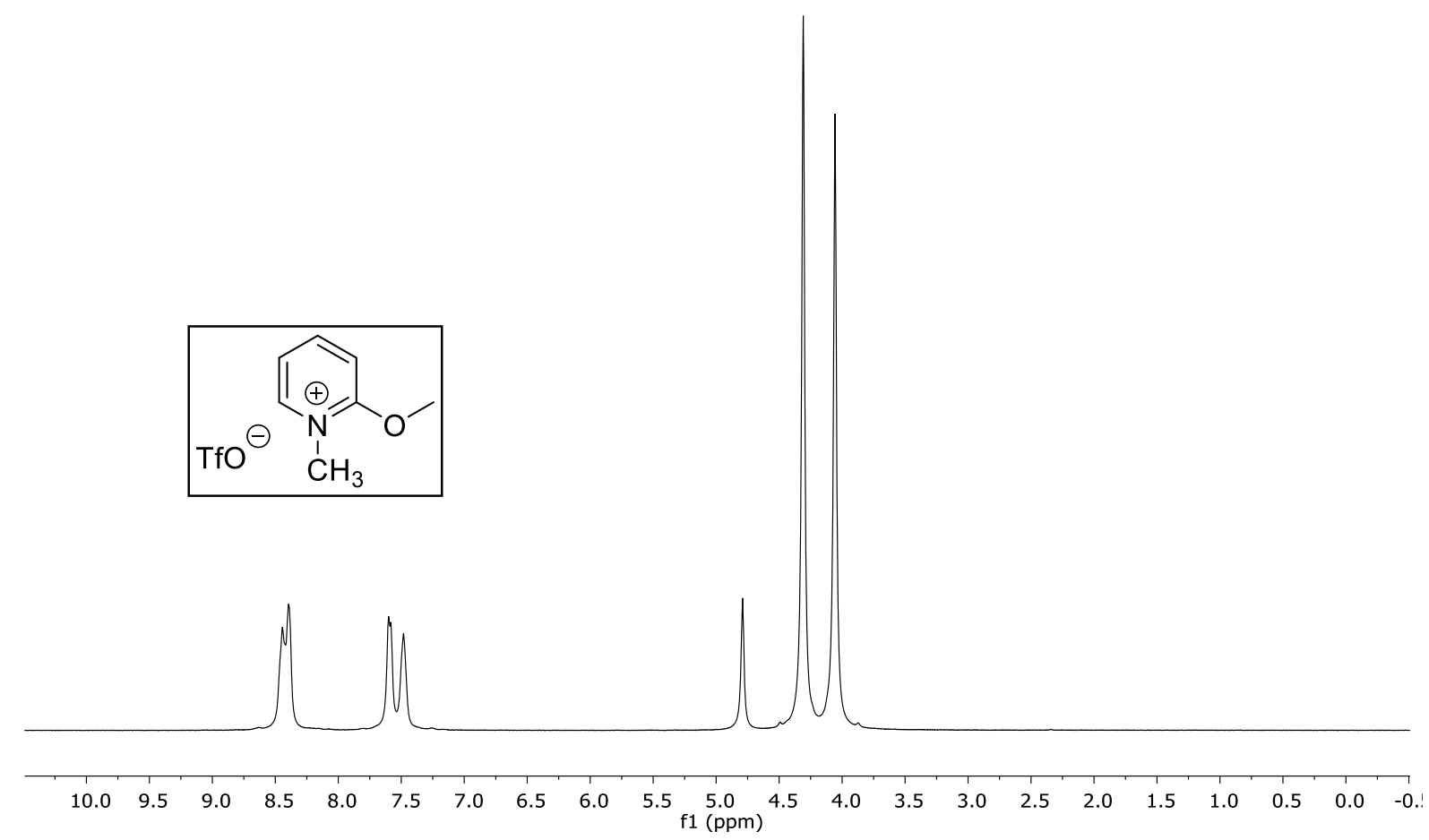

Compound 11b: ${ }^{13} \mathrm{C}$ NMR (101 MHz, $\left.\mathrm{D}_{2} \mathrm{O}\right)$

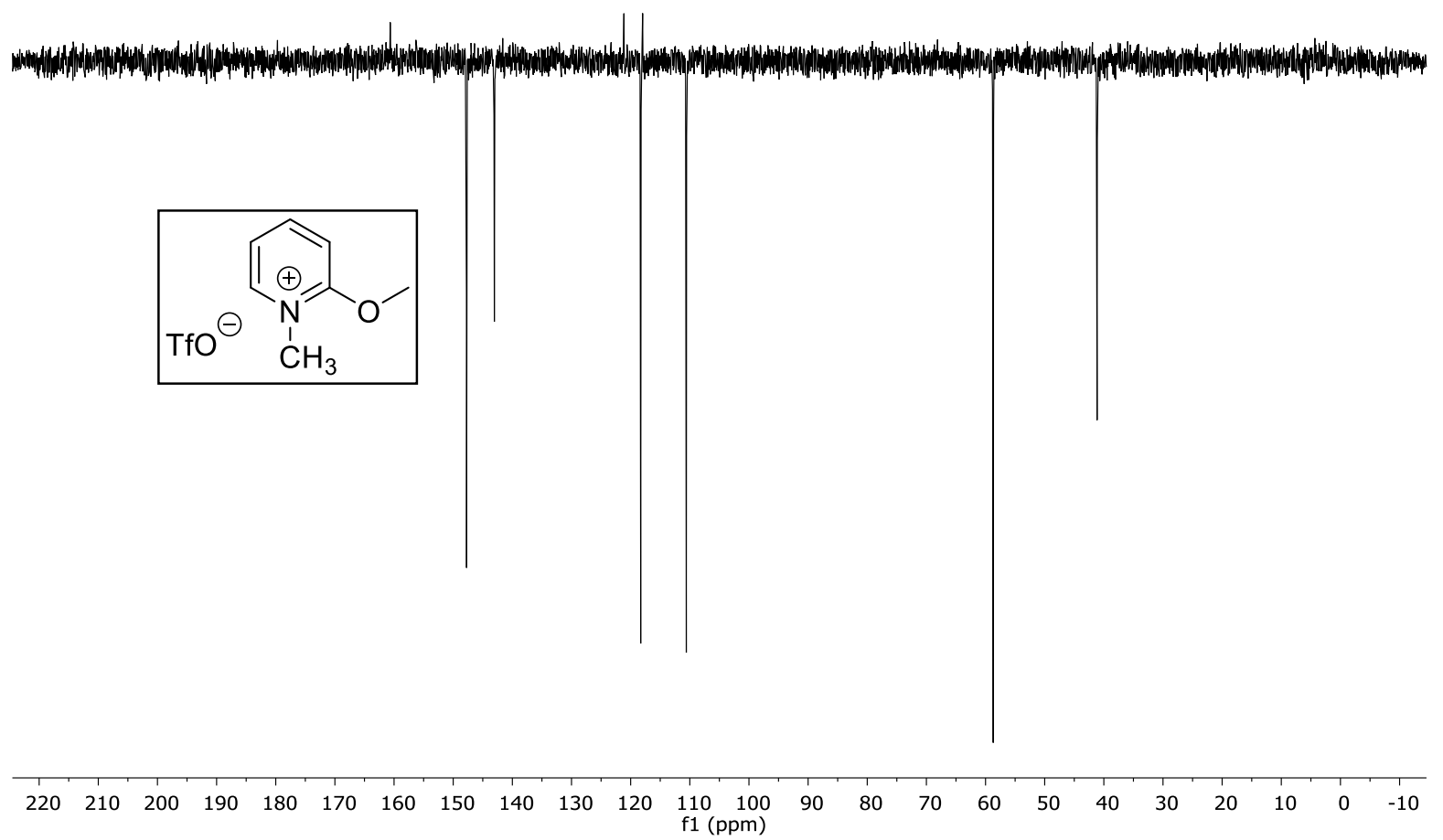


Compound 11c: ${ }^{1} \mathrm{H}$ NMR (400 MHz, $\left.\mathrm{D}_{2} \mathrm{O}\right)$
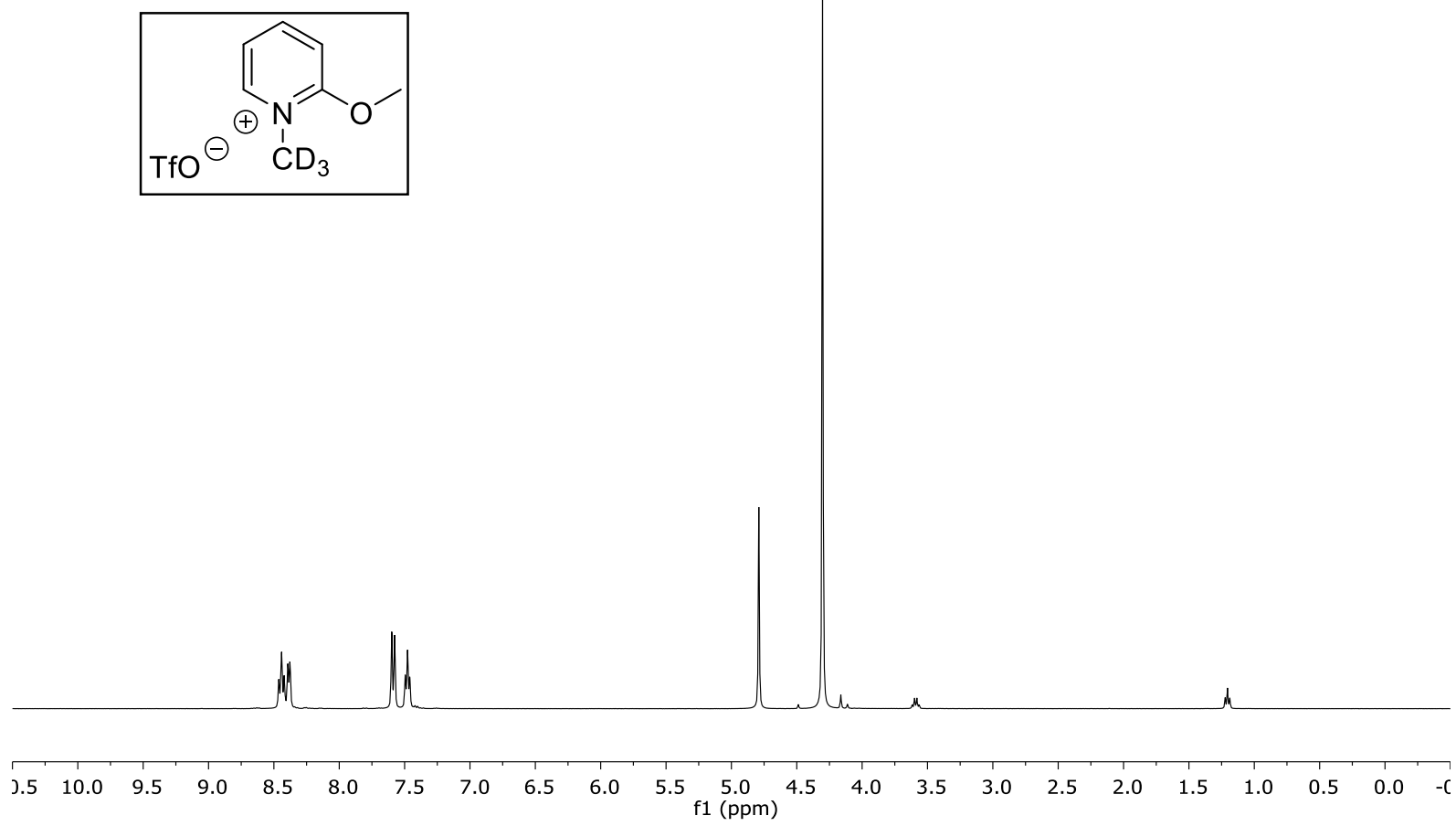

Compound 11c: ${ }^{13} \mathrm{C}$ NMR (101 MHz, $\left.\mathrm{D}_{2} \mathrm{O}\right)$

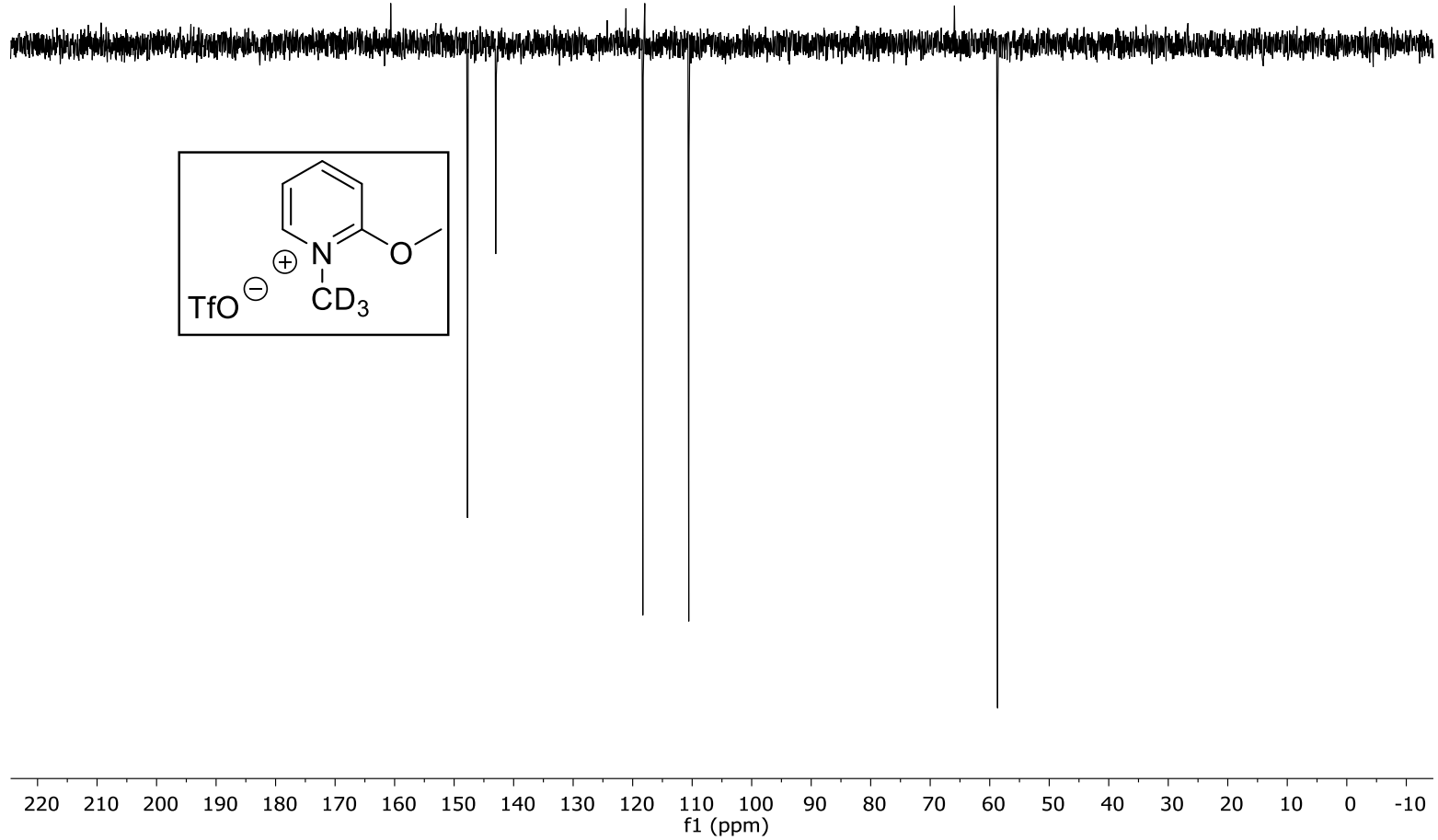




\section{References}

(1) Di Rienzo, B., Mellini, P., Tortorella, S., De Vita, D., and Scipione, L. (2010) Facile and Efficient Synthesis of 4-Alkyl Derivatives of 3-Carbamoyl- and 3,5-Dicarbamoylpyridines as Nicotinamide Mimetics. Synthesis (Stuttg). 2010, 3835-3838.

(2) Manaka, A., and Sato, M. (2005) Synthesis of Aromatic Thioamide from Nitrile Without Handling of Gaseous Hydrogen Sulfide. Synth. Commun. 35, 761-764.

(3) Poon, K. W. C., and Dudley, G. B. (2006) Mix-and-heat benzylation of alcohols using a bench-stable pyridinium salt. J. Org. Chem. 71, 3923-3927. 